\title{
WestVirginiaUniversity
}

THE RESEARCH REPOSITORY @ WVU

Graduate Theses, Dissertations, and Problem Reports

2005

\section{Stationary phase induction of RpoS in enteric bacteria}

Matthew Louis Hirsch

West Virginia University

Follow this and additional works at: https://researchrepository.wvu.edu/etd

\section{Recommended Citation}

Hirsch, Matthew Louis, "Stationary phase induction of RpoS in enteric bacteria" (2005). Graduate Theses, Dissertations, and Problem Reports. 2243.

https://researchrepository.wvu.edu/etd/2243

This Dissertation is protected by copyright and/or related rights. It has been brought to you by the The Research Repository @ WVU with permission from the rights-holder(s). You are free to use this Dissertation in any way that is permitted by the copyright and related rights legislation that applies to your use. For other uses you must obtain permission from the rights-holder(s) directly, unless additional rights are indicated by a Creative Commons license in the record and/ or on the work itself. This Dissertation has been accepted for inclusion in WVU Graduate Theses, Dissertations, and Problem Reports collection by an authorized administrator of The Research Repository @ WVU.

For more information, please contact researchrepository@mail.wvu.edu. 


\title{
Stationary Phase Induction of RpoS in Enteric Bacteria
}

\author{
Matthew Louis Hirsch \\ Dissertation submitted to the School of Medicine at \\ West Virginia University \\ In partial fulfillment of the requirements for the degree of \\ Doctor of Philosophy \\ in \\ Microbiology, Immunology and Cell Biology \\ Thomas Elliott Ph.D, Chair \\ Nyles Charon Ph.D \\ Lisa Salati Ph.D. \\ Rosana Schafer Ph.D. \\ Dave Yelton Ph.D.
}

Department of Microbiology, Immunology and Cell Biology

Morgantown, West Virginia

2005

Keywords: RpoS, ppGpp, Fis, translational regulation, Salmonella, Escherichia

Copyright 2005 Matthew L. Hirsch 


\section{Abstract: Stationary Phase Induction of RpoS in Enteric Bacteria}

\section{Matthew Louis Hirsch}

In enteric bacteria, stress adaptation is mediated by the RpoS protein, one of several sigma-factors that in association with RNA polymerase, collectively allow a tailored transcriptional response to environmental cues. Stress stimuli including low temperature, osmotic shock, and starvation all result in a substantial increase in RpoS abundance. Perhaps the most pronounced affect is observed during growth to stationary phase (SP) in rich medium. The mechanism of regulation depends on the specific signal, but may occur at the level of transcription, translation, protein activity or targeted proteolysis. In both Escherichia coli and Salmonella enterica cultured in rich undefined medium, the RpoS protein is barely detectable during exponential growth and increases $>30$-fold as cells enter SP. Under these conditions, SP induction depends on transcriptional and translational control with proteolysis affecting basal levels but not regulation per se. The transiently expressed Fis protein, whose abundance inversely correlates to that of RpoS, binds just upstream of the primary rpoS promoter and represses transcription nearly 10 -fold specifically during exponential growth. SP induction at the translational level relies on a novel form of genetic control dependent on the 24 nucleotides preceding the rpoS initiation codon (ribosome-binding sequence, RBS). The RNA secondary structure of the rpoS RBS is necessary and sufficient for a nearly 10 -fold translational increase during SP. Control at this level is not a result of differential transcript stability, nor does it involve the known rpoS regulators ppGpp, DksA, HU, Hfq or the small regulatory RNAs, DsrA and RprA. The environmental stimuli that trigger RBS-mediated SP induction of rpoS translation also remain unknown, but similar to transcriptional control, regulation is only seen in rich undefined media. Collectively, transcriptional repression by Fis and RBS-mediated 
induction at the translational level account for approximately $95 \%$ of the overall SP induction of RpoS. 


\section{Acknowledgments}

It's strange how life guides you to different places, for instance, six years ago I would have guessed that I would currently be in a medical residency program. Instead, I am finishing up my foundation as a scientific investigator. I do not question my current position because it led me to the great classic geneticist Tom Elliott, figuratively, among the last of a dying breed. By always "telling me what I needed to hear" (often repeatedly) he has provided me with the understanding and confidence to succeed in research. Additionally, I appreciate the evening conversations with Nyles Charon and Dave Yelton and the technical support of Adam Goodwill.

Socially, my friends and family have weaved a strong web of support and believe something fruitful will come of my work. Specific thanks to Jackie Bower, mom and dad, sister Shel, Aaron Marshall and Dev Jana, and the newcomers Bijal Patel, James Cunningham, and Amy Jones. You guys inspire me. And of course, my acknowledgment is not complete without recognition of the creature that probably has taught me the most in the least time, Neo the Wonderdog. 


\section{Table of Contents}

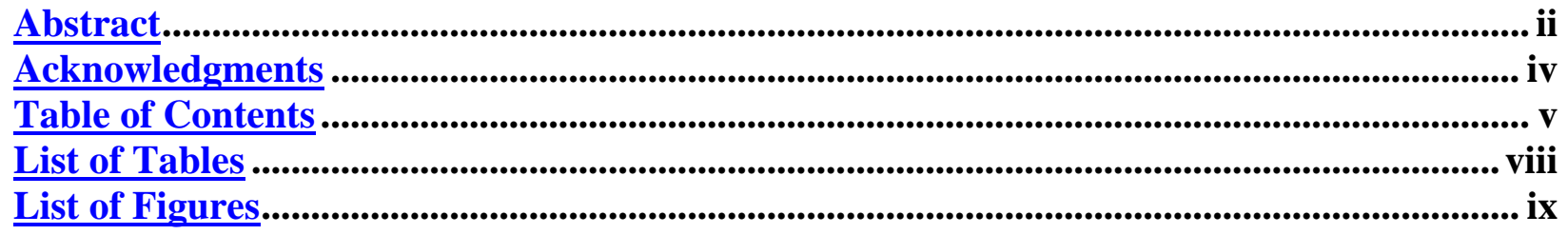

\section{Chapter 1}

Literature Review …….................................................................................................................................. 1

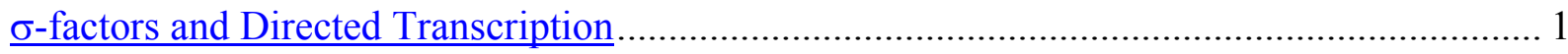

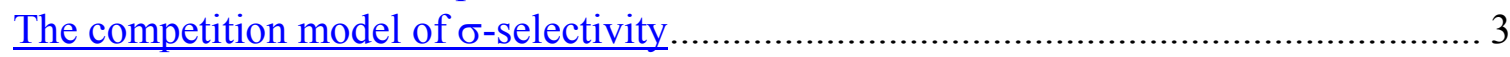

Promoter elements and $\sigma$-Selectivity........................................................................ 3

RpoS

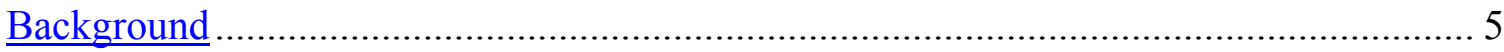

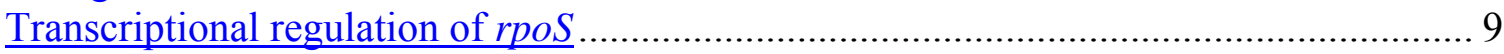

Translational regulation of RpoS ......................................................................... 14

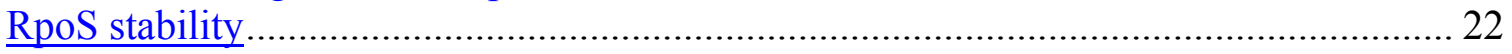

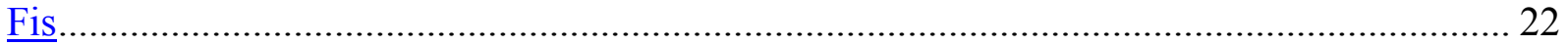

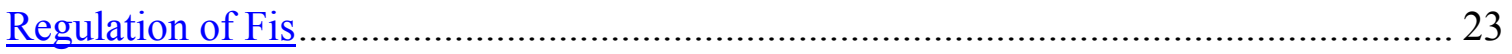

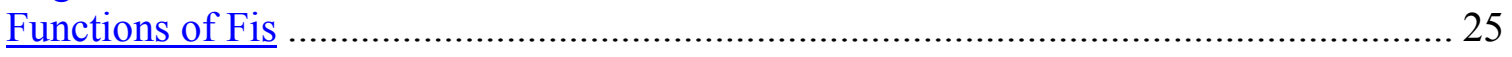

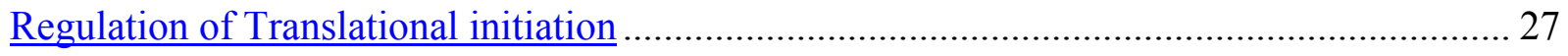

Shine-Dalgarno sequence and translational regulation....................................................29

Role of the initiation codon ……………................................................................. 30

Structured Ribosome Binding Regions.................................................................................. 31

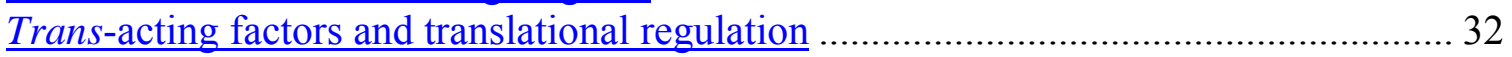

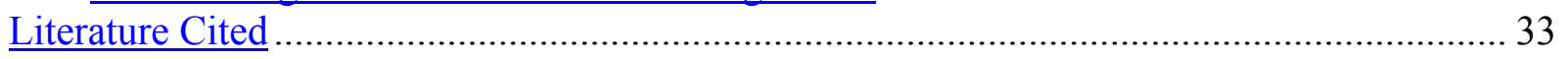

\section{Chapter 2}

Role of ppGpp in rpoS Stationary Phase Regulation in Escherichia coli ................................ 53

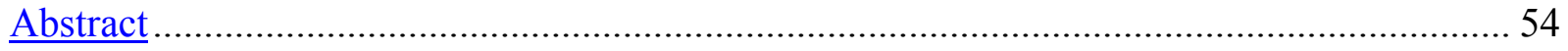

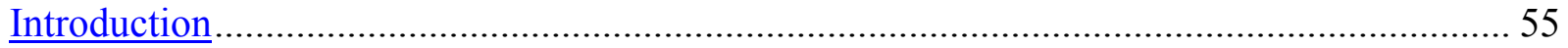

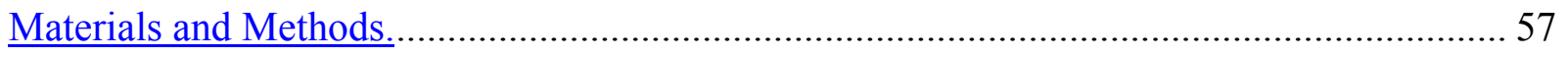

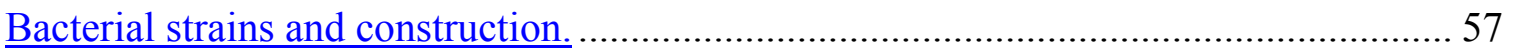

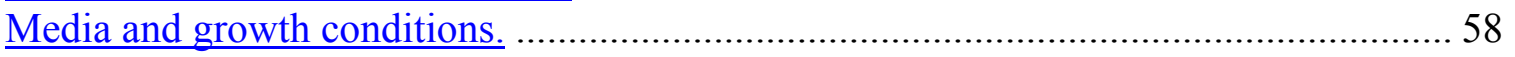

New mutations affecting putative trans-acting factors...................................................... 58

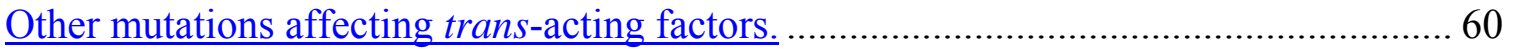

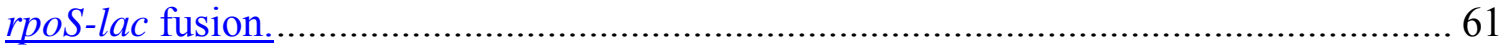

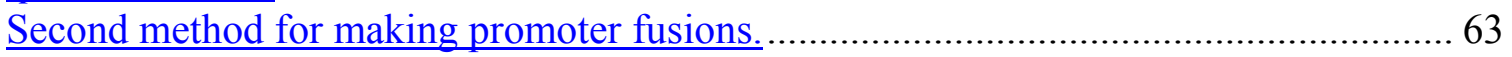

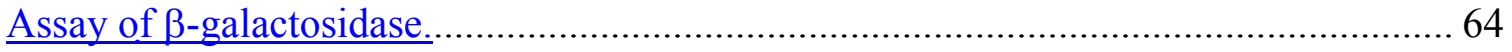

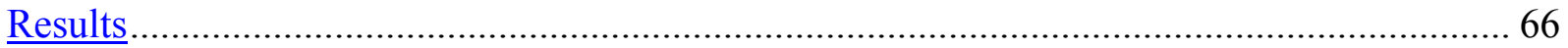

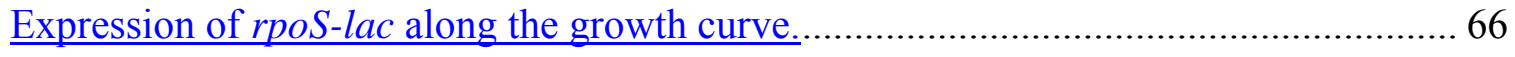




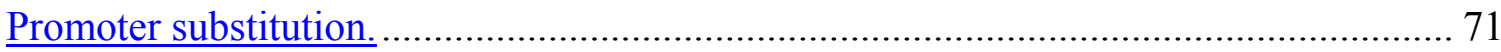

Deletion mutants.

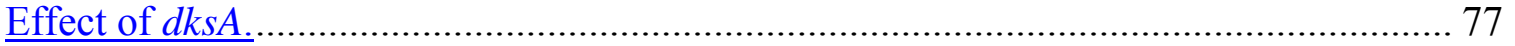

Stationary phase regulation localized to the rpoS RBS region........................................ 82

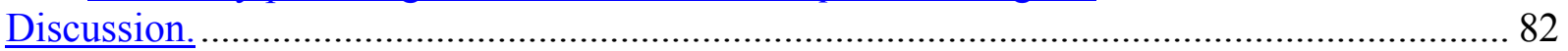

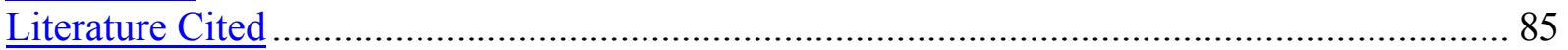

\section{Chapter 3}

Fis regulates transcriptional induction of RpoS in Salmonella enterica ...................................90 90

Abstract......................................................................................................................................................... 91

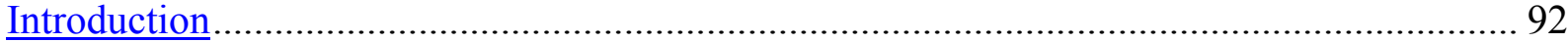

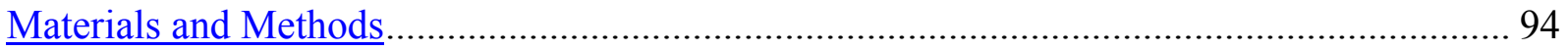

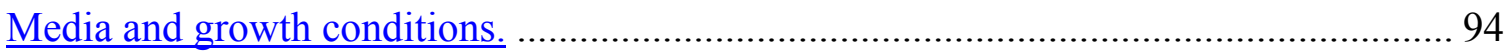

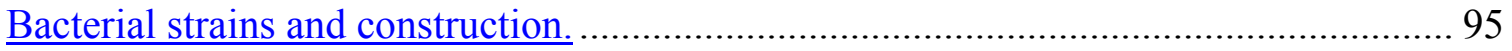

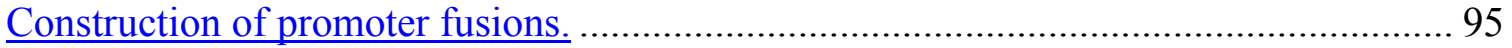

Construction of deletion / insertions and point mutations. ................................................ 96

Isolation of rpoS::MudJ insertion and construction of promoter deletions. …………....... 97

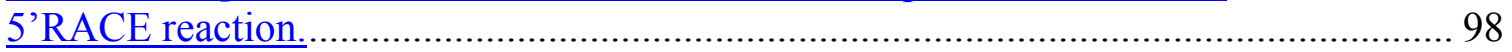

Immunological detection of proteins. ....................................................................... 99

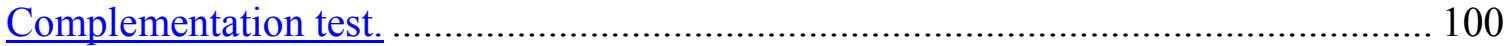

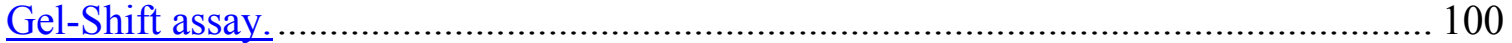

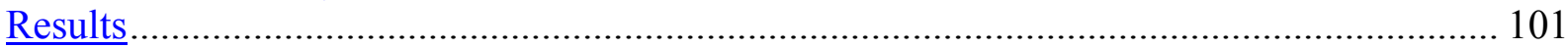

$\underline{\mathrm{SP}}$ induction of RpoS in S. enterica is normal even in the absence of regulated

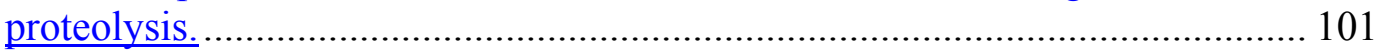

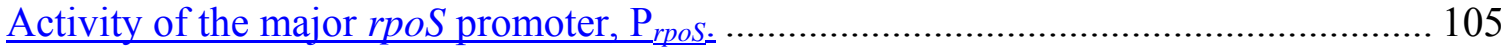

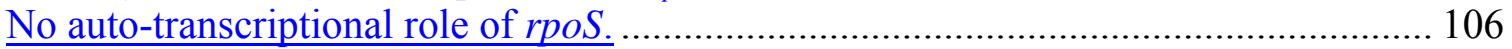

Computational analysis of the $r p o S$ promoter. …………............................................. 108

$\underline{\mathrm{P}}_{\underline{r p o s}}$ expression and RpoS protein level are elevated in a fis mutant. ............................ 110

Elevation of $\mathrm{P}_{\text {rpos }}$ activity in a fis mutant depends on a predicted Fis binding site. ....... 113

Fis protein binds to the $\mathrm{P}_{\text {rpos }}$ region. ……................................................................ 114

Testing the interaction of CRP and Fis in transcriptional regulation of rpoS................. 115

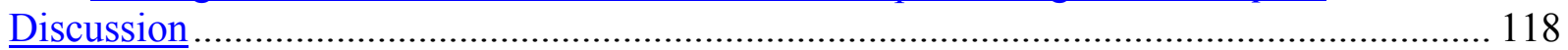

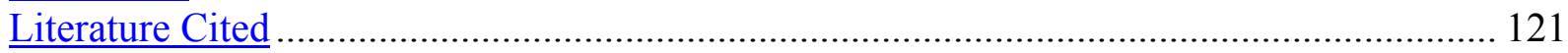

\section{Chapter 4}

Stationary Phase Regulation of RpoS synthesis in Escherichia coli........................................ 129

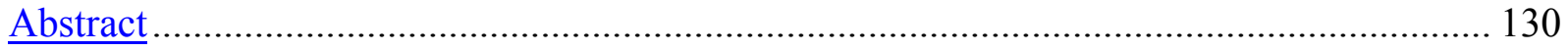

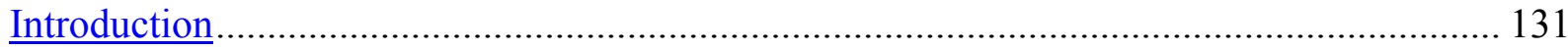

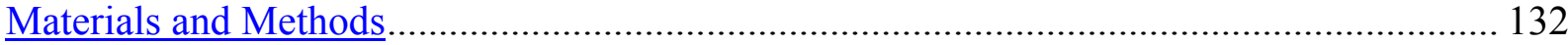

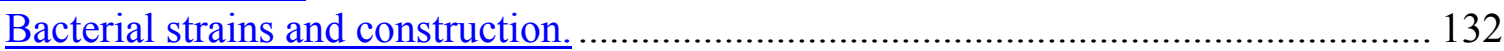

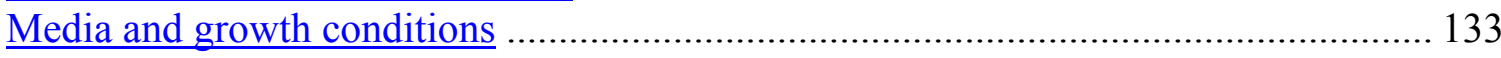

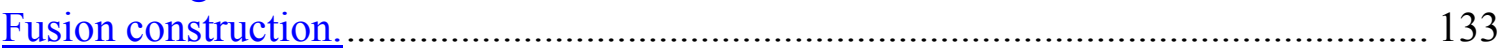

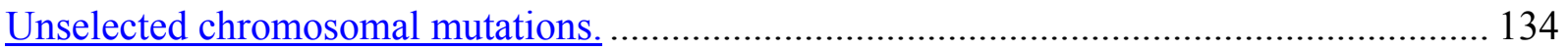




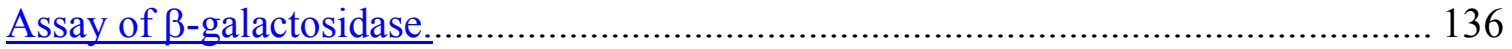

Immunological detection of proteins. …………........................................................ 136

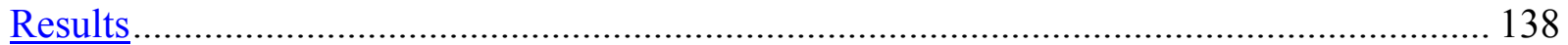

The rpoS RBS regulates SP induction of translation.................................................... 138

Conservation of rpoS RBS-mediated induction............................................................ 139

Dissection of the RBS and its role in SP induction. .......................................................... 140

Testing the role of potential trans-regulators in SP induction ....................................... 142

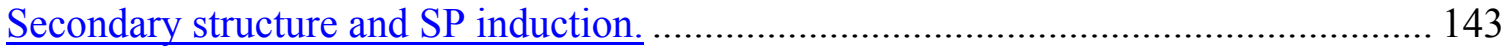

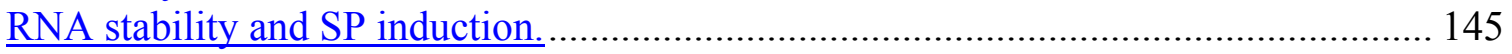

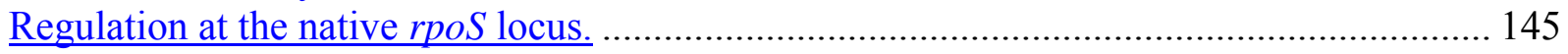

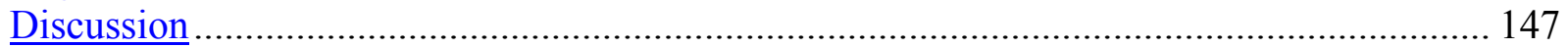

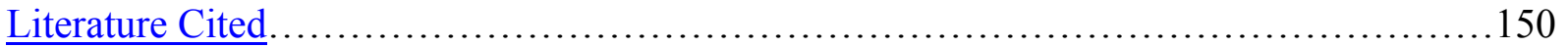

\section{$\underline{\text { Bacterial strains }}$}

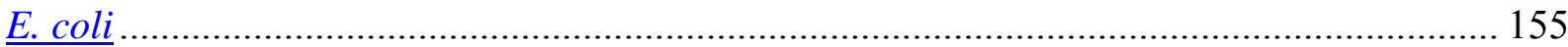

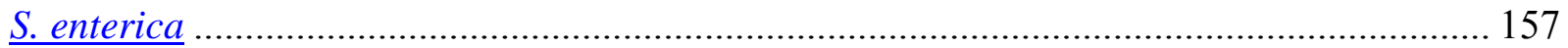

\section{$\underline{\text { Chapter } 5}$}

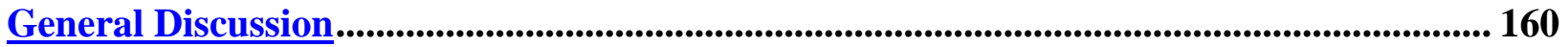

SP induction of RpoS in rich medium- The model ............................................................. 163

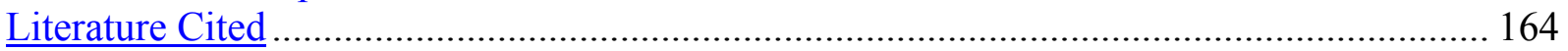




\section{List of Tables}

\section{Chapter 1}

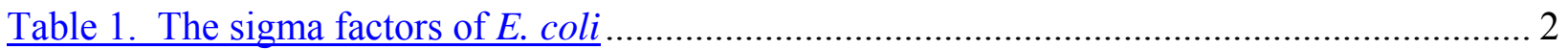

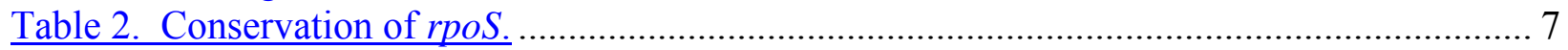

Table 3. Stimuli that increase RpoS protein abundance. ......................................................... 8

Table 4. Regulatory RNA that effect rpoS translation in E. coli ................................................... 17

\section{Chapter 2}

Table 5. Effect of ppGpp on rpoS-lac and lac expression in vivo ……..................................... 69 Table 6. Role of the antisense element in stationary phase induction and ppGpp effects on rpoS-

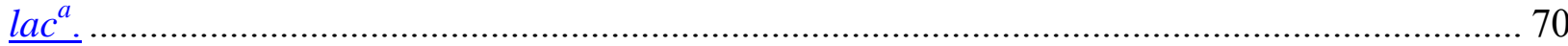

\section{Chapter 3}

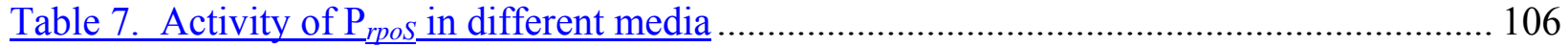

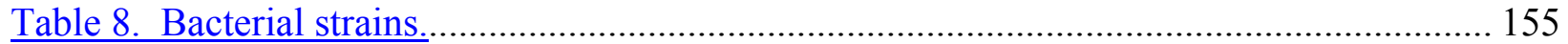




\section{$\underline{\text { List of Figures }}$}

\section{Chapter 1}

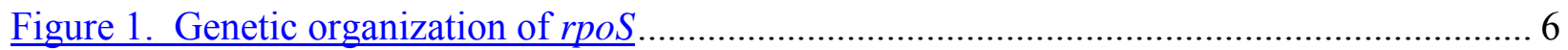

Figure 2. RNA secondary structure prediction of the rpoS leader region. ………...................... 15

\section{Chapter 2}

Figure 3. Partial restriction map of the $n l p D$ and $r p o S$ open reading frames. ........................... 65

Figure 4. Expression of rpoS-lac [pr] as a function of growth phase........................................ 67

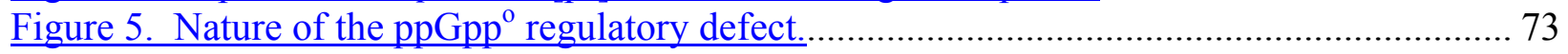

Figure 6. Deletion mapping of sequences required for $\mathrm{ppGpp}^{\circ}$................................................... 75

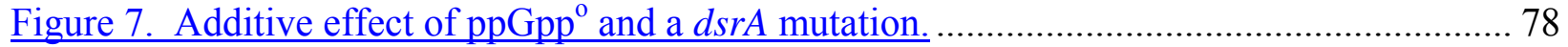

Figure 8. Stationary phase regulation requires the wild type rpoS RBS. .................................... 81

\section{Chapter 3}

Figure 9. SP induction of RpoS is normal in turnover-defective mutants.................................. 101

Figure 10. Promoter activity analyzed using rpoS: MudJ as a reporter. .................................... 103

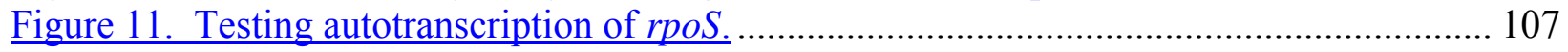

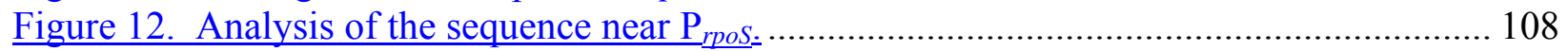

Figure 13. RpoS protein is elevated in a fis null mutant........................................................ 109

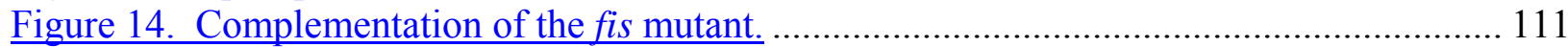

Figure 15. Transcriptional regulation of rpoS by Fis depends on Fis site III. ........................... 112

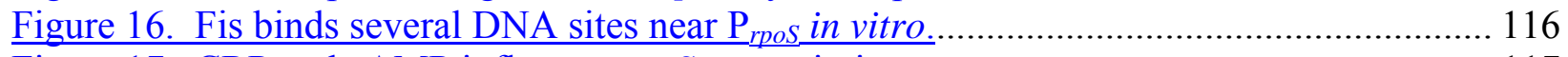

Figure 17. CRP and cAMP influence $r p o S$ transcription...................................................... 117

\section{Chapter 4}

Figure 18. Stationary phase induction of rpoS translation relies on the ribosome-binding

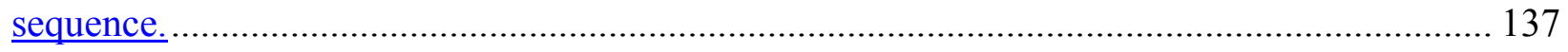

Figure 19. $\beta$-galactosidase activity of various ribosome-binding sequences. ........................... 140

Figure 20. Predicted RNA secondary structure of the rpoS ribosome-binding region............... 143

Figure 21. Stationary phase regulation of various ribosome-binding sequences at the .............. 144

Figure 22. SP regulation of RpoS depends on the rpoS RBS and Fis. ...................................... 146 


\section{Literature Review}

\section{$\sigma$-factors and Directed Transcription}

The ability to sense unfavorable growth environments and coordinate appropriate gene expression allows the survival of bacteria in nature. External stimuli adjust the abundance of global regulators that participate in signaling networks culminating in specific or general stress adaptation. Primary levels of regulatory input include transcription, translation, protein activity and protein degradation.

Transcriptional control is particularly important because the signal is amplified, each RNA molecule can generate thousands of protein molecules. In bacteria, RNA polymerase core enzyme (RNAP) catalyzes the synthesis of RNA using DNA as the reaction template. However, RNAP by itself is without direction and initiates transcription at aberrant sites including nicked or "open" regions of DNA (23). In E. coli and S. enterica one of seven additional factors, termed sigma $(\sigma)$-factors, bind RNAP (the complex is termed RNAP holoenzyme) and confer promoter recognition of specific groups of genes (termed regulons; 116). These $\sigma$-factors are listed in Table 1 along with their abundance during exponential growth, their RNAP dissociation constants, and the general function of the genes they regulate. Of particular importance are the two $\sigma$-factors that direct most gene expression under different growth conditions, RpoD and RpoS. RpoD is the most abundant $\sigma$-factor in dividing cells and directs RNAP to promoters of genes necessary for optimal growth. During this time, RpoS is barely detectable, but protein abundance dramatically increases in response to unfavorable growth conditions (i.e. starvation, 
low $\mathrm{pH}$, high osmolarity; reviewed in ref. 61). Through transcriptional control of a large regulon, RpoS orchestrates the general stress response in which there is a reversible transition from exponential growth to a non-dividing stress-resistant state called stationary phase (SP).

Table 1. The sigma factors of $E$. coli

\begin{tabular}{lccl}
\hline$\sigma$-factor & $\begin{array}{c}\mathbf{K}_{\mathbf{d}}(\mathbf{n M}) \\
(\mathrm{RNAP}-\sigma)\end{array}$ & $\begin{array}{c}\text { Intracellular } \\
\text { Concentration } \\
\text { (molecules/cell) }\end{array}$ & \multicolumn{1}{c}{$\begin{array}{c}\text { Genes } \\
\text { Activated }\end{array}$} \\
\hline$\sigma^{70}(\mathrm{RpoD})$ & 0.26 & 700 & growth related/housekeeping \\
$\sigma^{54}(\mathrm{RpoN})$ & 1.55 & 110 & nitrogen utilization \\
$\sigma^{38}(\mathrm{RpoS})$ & 4.26 & $<1$ & general stress \\
$\sigma^{32}(\mathrm{RpoH})$ & 1.24 & $<10$ & heat shock response \\
$\sigma^{28}(\mathrm{RpoF})$ & 0.74 & 370 & flagellar synthesis; chemotaxis \\
$\sigma^{24}(\mathrm{RpoE})$ & 2.43 & $<10$ & extracytoplasmic/heat shock \\
$\sigma^{\text {Fecl }}$ & 1.73 & $<1$ & ferric citrate transport \\
\hline \hline
\end{tabular}

The sigma $(\sigma)$-factors of $E$. coli are listed with their RNAP dissociation constants $\left(\mathrm{K}_{\mathrm{d}}\right)$ determined by core enzyme binding in mixed reconstitution experiments (90). The intracellular concentration of each factor during exponential growth is also presented along with the general function of each regulon $(69,70,98,99)$.

Regulation of $\sigma$-directed transcription has two primary components. First, $\sigma$-factors compete for a limited amount of RNAP, a process biased by their relative abundance and individual binding affinities for RNAP (Table 1; reviewed in ref. 124). Second, $\sigma$-factors must display promoter specificity; an enigmatic process that requires sequence elements at targeted promoters and often, additional transcriptional factors and physiological indicators $(83,99)$. Directed transcription by RpoS and RpoD is discussed below. 


\section{The competition model of $\sigma$-selectivity}

The competition model for RNAP states that $\sigma$-factors compete for a limited amount of core RNAP and this in turn, contributes to targeted gene expression (42). During SP, RpoSdependent expression of numerous genes occurs despite a 16-fold affinity deficit for RNAP compared to that of the housekeeping factor, RpoD (98). Experiments employing lacZ fusions to promoters expressed during SP determined that a mild overproduction of RpoD during SP eliminates RpoS-dependent gene expression; conversely, overproduction of RpoS reduces RpoDdependent gene expression (42). These results correlate with RpoS abundance during different stages of growth; RpoS is low during expression of most RpoD-dependent promoters (exponential growth) and high during SP (70). Simplistically, the sizeable SP induction of RpoS abundance overcomes its affinity deficit for RNAP, resulting in RpoS-dependent gene expression $(42)$.

\section{Promoter Elements and $\sigma$-Selectivity}

After a $\sigma$-factor successfully binds to core RNAP, it then confers promoter selectivity, a process, mainly defined by promoter specific elements. This is explicit in the conserved sequences of promoters individually recognized by the alternative $\sigma$-factors RpoN, RpoH, RpoF, RpoE or FecI (99). For example, RpoF-RNAP only transcribes genes involved in chemotaxis and the synthesis of flagella with promoter recognition defined by specific nucleotides (nt) of the -10 and -35 hexamers exhibited by RpoF-dependent promoters (83). 
The recognition of specific promoters by RpoD and RpoS, however, is complicated by very similar consensus sequences and in general, is not completely understood (48). In search of a RpoS consensus sequence, regions preceding a core set of 140 RpoS-dependent genes induced regardless of the stress challenge, were analyzed in silico (178). The results identified conservation of an $11 \mathrm{nt}$ sequence (5'-TCTATACTTAA-3') that strongly resembles an extended -10 promoter hexamer $(61,178)$. Additional RpoS-dependent promoters of genes expressed in response to a specific challenge exhibited no sequence similarities (178).

Tanaka et al. investigated RpoS and RpoD transcription specificity in vitro and defined three classes of promoters: (i) promoters recognized by RpoD or RpoS at equal efficiencies (e.g. lacUV5 and trp), (ii) RpoD-dependent promoters (e.g. ribosomal and tRNA genes) and (iii) a promoter that prefers RpoS-RNAP holoenzyme (fic) (165). Alignment of promoters from each class did not identify any differences between class I and II promoters, while class III promoters generally lacked a typical -35 sequence (165). These results are consistent with the notion that promoter selectivity by RpoS may be achieved by degenerate RpoD promoter elements in conjunction with additional regulatory factors (48).

Intracellular ionic conditions and other physiological indicators also affect RpoD and RpoS promoter recognition $(38,76,85)$. RpoS-dependent promoters expressed during SP were transcribed in vitro by both RpoS and RpoD (76). RpoD promiscuity was eliminated by the addition of high concentrations of glutamate salts, a condition reported to mimic intracellular ionic conditions under hyperosmotic stress $(38,76)$. Physiological indicators of SP physiology, 
including trehalose, guanosine tetraphosphate (ppGpp), or a decrease in DNA superhelical density, also favor RpoS over RpoD in promoter selection antagonism $(29,71,85,86)$.

Global transcription factors that influence $\sigma$-selectivity at target promoters include the catabolite repressor protein (CRP), the integration host factor (IHF) and the leucine response protein (Lrp; 32). For example, both RpoD and RpoS initiate transcription at the osmY promoter $\left(\mathrm{P}_{\text {osmY }}\right)$ in vitro, but addition of the in vivo regulators CRP, IHF, and Lrp disproportionately repressed RpoD-directed transcription (32). Collectively, promoter recognition by RpoD and RpoS is influenced by many factors including promoter sequence elements, ionic conditions, trehalose concentrations, DNA topology, ppGpp, and protein regulators.

\section{RpoS}

\section{Background}

During the 1980's, the rpoS gene was identified by several labs for roles in sensitivity to

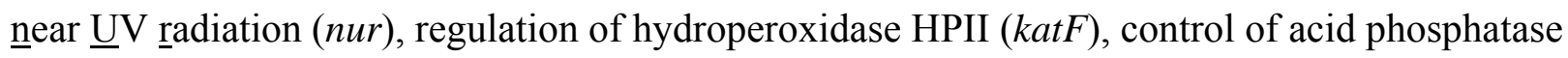
activity $(a p p R)$, and carbon starvation (csi-2; 61). Similar phenotypes of the mutants as well as genetic mapping confirmed that nur, appR, katF, csi-2 were in fact different names for the same gene $(90,167)$. Mulvey and Loewen reported the sequence of this gene, originally as katF (for its role in catalase HPII synthesis), revealing an 1086 base pair (bp) open reading frame that encodes a 362 amino acid protein (112). The amino acid sequence demonstrates strong similarity to the housekeeping $\sigma$-factor RpoD, including the region required for binding RNAP. 
Therefore, in 1991 Lange and Hengge-Aronis renamed it RpoS, after the RpoD-related protein family and its involvement in stress adaptation (90). Two years later, Tanaka et al. confirmed that RpoS is indeed a $\sigma$-factor for RNAP (165). In that work, RpoS-RNAP holoenzyme transcribed several RpoD-dependent promoters and one of them (fic) was favored by RpoS, suggesting altered promoter recognition specificity (165).

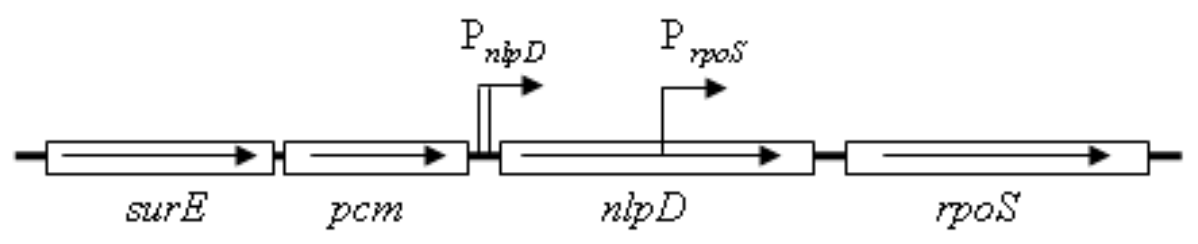

Figure 1. Genetic organization of rpoS

The rpoS region of the E. coli chromosome (minute 61.8 to 61.7) is depicted with straight arrows indicating transcriptional polarity. Promoters contributing to rpoS expression are shown with bent arrows $(87,126,164)$.

The rpoS gene is highly conserved among the $\gamma$-branch of proteobacteria (Table 2) and its genetic organization is depicted in Figure 1. rpoS is located downstream of genes whose products are also involved in aspects of stress resistance: SurE (a novel phosphatase), Pcm (Lisoaspartyl methyltransferase), and NlpD (a lipoprotein with suggested hydrolytic functions in cell wall maintenance; $47,65,88,94)$. Transcriptional induction of both surE and pcm occurs in response to stress in a RpoS-independent manner (95). 
Table 2. Conservation of rpoS.

\begin{tabular}{lc}
\hline Bacteria & $\begin{array}{c}\text { Nucleotide } \\
\text { Conservation }\end{array}$ \\
\hline S. typhimurium & $100 \%$ \\
S. typhi & $99 \%$ \\
E. coli MG1655 & $92 \%$ \\
E. coli 0157:H7 & $92 \%$ \\
Shigella flexneri & $92 \%$ \\
Enterobacter cloacae & $89 \%$ \\
Kluyvera cryocrescens & $84 \%$ \\
Serratia entomophila & $83 \%$ \\
Erwinia carotovora & $83 \%$ \\
Yersinia pestis & $80 \%$ \\
\hline a The percent nucleotide conservation of the \\
rpoS coding sequence, relative to S. enterica \\
Typhimurium, is shown.
\end{tabular}

\section{RpoS function and regulon}

RpoS has been deemed the "master regulator of the general stress response" due to its transcriptional control of over $10 \%$ of the E. coli genome in response to different types of stress $(61,67,178)$. The gene products of the RpoS regulon have diverse functions under the general heading of stress survival $(61,178)$. Of these, transcriptional profiling has identified a core set of 140 genes activated in response to acid shock, osmotic shock, or growth to SP (178). In addition, hundreds of other RpoS-dependent genes are specifically expressed in response to a particular type of stress, allowing a tailored response (178). Stress stimuli that influence RpoS abundance, along with their reported levels of regulatory input, are listed in Table 3. 
Table 3. Stimuli that increase RpoS protein abundance.

\begin{tabular}{lll}
\hline Stimulus & Level of Regulation & Reference \\
\hline \multirow{2}{*}{ Slow growth rate } & Transcription & $(61)$ \\
& Translation & $(34)$ \\
Growth to SP & Transcription & $(88)$ \\
& Translation & $(88)$ \\
Low temperature & Translation & $(158)$ \\
High osmolarity & Translation & $(111)$ \\
Carbon starvation & Protein Stability & $(111)$ \\
Low pH & Protein Stability & $(88,130)$ \\
& Translation & $(61)$ \\
Heat Shock & Protein Stability & $(12)$ \\
\hline
\end{tabular}

Bacteria containing a mutation in $r p o S$ exhibit increased sensitivity to a variety of stress conditions, most of which are encountered during mammalian infection (61). These challenges include single nutrient deprivation, outright starvation, oxidative stress, high osmolarity, acid stress, and DNA damage - all of which normally stimulate RpoS induction conferring resistance $(41,61,106)$. The characteristic changes in morphology during stress, cells become smaller and spherical, are abolished in the rpoS mutant (89). In addition, mutants do not accumulate glycogen, the storage form of glucose, or the disaccharide trehalose, which normally accumulates during SP and plays an undefined role in stress survival $(62,86,104)$.

\section{RpoS and Salmonella Infection models}

RpoS plays an essential role in virulence. Perhaps this is most explicit in a mouse model wherein infection by Salmonella enterica serovar Typhimurium results in a typhoid-like disease 
that causes death within days (41). In this case, mice infected by rpoS mutants survived (41). This dramatic result is due to a combination of decreased colonization of the gut-associated lymphoid tissue and decreased expression of the RpoS-dependent virulence plasmid, spv (30, 41, 118, 180). In humans, rpoS mutants of S. enterica serovar Typhi do not cause disease. In fact, the live oral typhoid vaccine Ty21a is attenuated for virulence due primarily to a null mutation in $\operatorname{rpoS}(137)$.

In contrast, RpoS appears dispensable for Salmonella infection of either macrophages or epithelial cell cultures $(118,180)$. Infection of these cells by S. enterica Typhimurium causes a five to 10-fold increase in RpoS expression and RpoS-dependent reporter activity, including genes involved in virulence (31). However, rpoS mutants show wild type levels of attachment, invasion, and survival in the phagosomal compartment during infection of macrophages and intestinal epithelial cells $(118,180)$. Further investigations performed in mice demonstrate that RpoS is necessary for bacterial adherence of the Peyer's patches (118). These results demonstrate that current in vitro infection models do not reflect the importance of RpoS for pathogenesis in vivo.

\section{Transcriptional regulation of $r p o S$}

Analysis of rpoS transcription in E. coli by primer extension, and in S. enterica serovar Dublin by both primer extension and Northern blot, established that rpoS is transcribed from two distinct promoter regions $(87,126,164)$. Two closely-spaced and relatively weak promoters 
(collectively termed $\mathrm{P}_{n l p D}$; Fig. 1) generate a bicistronic nlpD-rpoS message, while the major promoter $\left(\mathrm{P}_{r p o s}\right)$ is located approximately in the center of the $n l p D$ coding sequence and generates a monocistronic rpoS transcript with a 5' untranslated leader region of $565 \mathrm{nt}$ (87, 126). E. coli and $S$. enterica share identical -35 and -10 hexamers at $P_{r p o s}$ with a 17 bp spacer, and initiate transcription at the same nt $(87,126)$.

Regulation of transcription from $\mathrm{P}_{\text {rpos }}$ is a complex and poorly characterized phenomenon that is dependent upon the growth medium [i.e. Luria-Bertani (LB) vs. minimal medium]. When E. coli grows to $\mathrm{SP}$ in $\mathrm{LB}$, rpoS transcriptional fusions demonstrate a five to10-fold increase in activity $(88,90)$. In contrast, no SP induction of transcription occurs when cells grow in minimal medium containing glucose despite a dramatic increase in RpoS abundance (187). The global regulators that influence $r p o S$ transcription, $\mathrm{CRP}$, ppGpp, and inorganic polyphosphate (poly-P) are discussed in the following sections. However, other conditions may also influence rpoS transcription, such as weak acids or homoserine lactone, although reports are often conflicting $(61,64,154)$.

\section{CRP-cAMP and rpoS transcription}

Catabolite repression is a well established mechanism in most bacteria and some lower eukaryotes to allow the preferential use of the most energetically favorable carbon source (35, 80, 115). In E. coli and S. enterica, membrane-associated adenylate cyclase (encoded by the cya gene) catalyzes the reaction: ATP $\rightarrow$ cyclic AMP (cAMP) in response to low cellular 
concentrations of catabolites (reviewed in ref. 115). Increased levels of cAMP result in binding to and activation of CRP, a homodimer that interacts with DNA near target promoters. CRP dually functions as a transcriptional activator and repressor, ensuring the timely expression of a large regulon (>100 genes) which includes other global regulators such as the factor for inversion stimulation, Fis (53).

Surrounding $\mathrm{P}_{r p o s}$ are two predicted CRP-cAMP binding sites (the consensus half-site is TGTGAN $_{3}$ ); one of these is centered at a classical upstream (with respect to the transcriptional start site) activation position, while the downstream location of the other site suggests a role in repression (24). E. coli strains harboring mutations in either cya or crp demonstrate a modest increase in rpoS transcription during exponential phase $(88,90)$. The exogenous addition of cAMP to the cya mutant culture restored $\mathrm{P}_{r p o s}$ activity to wild type levels (88). In addition, a crrencoded EIIA(Glc) mutant, which lacks the soluble part of the phosphtransferase solute uptake system that activates adenylate cyclase, demonstrates elevated rpoS transcription (169). Collectively, these reports suggest CRP-cAMP mediated repression of $\mathrm{P}_{\text {rpos }}$ during exponential phase. Additional unpublished results of Hengge-Aronis suggest that CRP-cAMP also activates $\mathrm{P}_{r p o s}$ expression during the transition to SP, possibly through binding to the downstream site (61). 


\section{ppGpp and rpoS transcription}

Amino acid limitation or, more specifically, the failure to aminoacylate tRNAs results in pleiotropic physiological alterations that efficiently adjust gene expression to accommodate survival in the limited nutrient state. This cascade of events is generally termed the stringent response and is mediated by the alarmone ppGpp (115). As nutrients are depleted from the growth medium, increased levels of ppGpp fine-tune cellular physiology at the transcriptional level via interactions with RNAP holoenzyme (115). Sensibly, ribosomal RNA operons are among the genes inhibited by ppGpp, while genes necessary for amino acid biosynthesis and uptake are examples of those activated during the stringent response $(115,127)$.

ppGpp is synthesized by the ribosome-associated RelA protein, and to a lesser extent, by the cytosolic SpoT protein which also degrades $\operatorname{ppGpp}(27,49)$. The RelA protein senses amino acid limitation by evaluating the ratio of charged / uncharged tRNA molecules at the acceptor position of the ribosome (139). If a threshold of uncharged tRNA molecules is achieved, RelA converts GTP + ATP (through the unstable intermediate pppGpp) or GDP into ppGpp (115). Consequently, the concentration of ppGpp indicates the cell's nutritional status and its basal levels vary inversely with growth rate (115).

A crystal structure of the RNAP-ppGpp complex shows that binding occurs near the catalytic center of RNAP (5). This interaction is thought to block incoming nucleoside triphosphates and thereby decrease the half-life of open transcriptional complexes at DNA promoters $(9,55)$. Regulation at this level is especially effective at promoters that exhibit shortlived open complexes including those of ribosomal RNA operons (9). Stabilization of the 
ppGpp-RNAP interaction depends on DksA, a DNA-binding protein also implicated in posttranscriptional regulation of RpoS synthesis $(22,128)$.

ppGpp accumulation is often, if not always, accompanied by RpoS induction $(22,50,87)$.

Western blot experiments and both rpoS transcriptional and translational fusions demonstrate a dramatic reduction of RpoS expression in ppGpp deficient strains during both exponential growth and SP $(50,87)$. Consistently, artificial induction of ppGpp during exponential phase increased RpoS abundance approximately 50-fold (22). However, due to the pleiotropic nature of altered ppGpp levels, it is possible that its control of RpoS expression is indirect, and mediated via causal effects such as aberrant ribosomal RNA levels, decreased growth rate, or altered levels of inorganic polyphosphate (poly-P; 152).

\section{Inorganic Phosphate and rpoS transcription}

E. coli contain three types of inorganic phosphates, $\mathrm{Pi}, \mathrm{PPi}$ and poly-P, the concentrations of which independently vary in accordance with cellular physiology (reviewed in ref. 115). During exponential growth their relative concentrations follow the scheme $\mathrm{Pi}>>\mathrm{PPi}>$ poly-P (115). However during times of stress, polyphosphate kinase (encoded by ppk) utilizes the terminal phosphate of ATP to synthesize a long polymer of orthophosphate residues linked by high energy phosphoanhydride bonds (25). The importance of poly-P is emphasized by its ubiquitous nature in all forms of life examined (reviewed in ref. 182) and implicated functions 
include an ATP substitute for adenylate kinases (129), a phosphate reservoir (25), and a role in the acquisition of competence (28).

E. coli and S. typhimurium mutants devoid of Ppk function are more susceptible to a variety of stresses and do not survive long periods of $\operatorname{SP}(77,133)$. This is due, in part, to the influence of poly-P on rpoS transcription. E. coli cells lacking poly-P, due to gratuitous over expression of a yeast exophosphatase, are deficient in SP accumulation of RpoS (152). This result was primarily attributed to decreased $r p o S$ transcription, although the mechanism remains largely unresolved (152). To complicate matters, over expression of poly-P does not affect ppGpp levels, although increased concentration of the latter results in a massive accumulation of poly-P (up to 1000-fold; 84). It is therefore possible that the influence of ppGpp on rpoS expression is mediated via elevated levels of poly-P.

\section{Translational regulation of RpoS}

Transcripts originating from $\mathrm{P}_{r p o s}$ carry a $565 \mathrm{nt} 5$ ' untranslated region (leader region) preceding the rpoS initiation codon whose sequence is conserved among enteric bacteria. In particular, $110 \mathrm{nt}$ preceding the rpoS initiation codon, which includes a cis-acting antisense element that sequesters the $r p o S$ ribosome-binding sequence (RBS), transiently act to decrease translation (61). The timely disruption of the inhibitory structure in response to particular stress stimuli induces rpoS synthesis (61). Genetic evidence and in silico structure predictions agree on a secondary structure for this region of the rpoS leader (Fig. 2, nt 454-565; 33, 101, 193). 


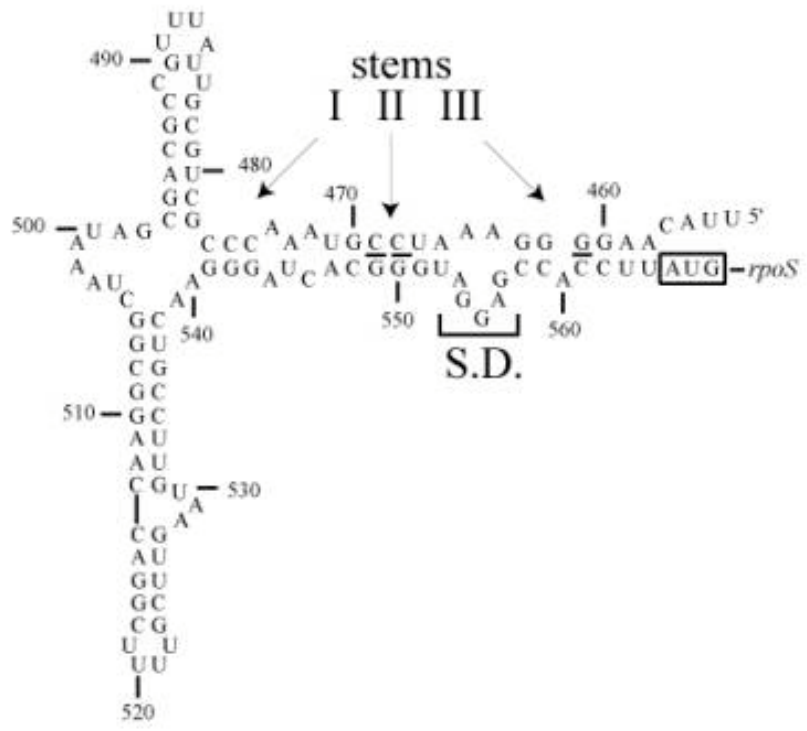

Figure 2. RNA Secondary Structure Prediction of the rpoS leader region.

A secondary structure prediction of 112 nucleotides of the rpoS leader region $(21,101)$. The numbering refers the nucleotide position of the rpoS transcript originating from $\mathrm{P}_{\text {rpos. }}$. The $r p o S$ initiation codon is outlined by a black box and the Shine-Dalgarno sequence is labeled S.D.. Three stems of the antisense element are designated I-III.

The physical structure(s) of the $r p o S$ leader is not established conclusively, although a deletion analysis and site-directed mutations employing rpoS-lacZ translational fusions are consistent with an inhibitory role of the leading prediction (Fig. 2; 33, 101). A rpoS translational fusion containing both stems II and III (Fig. 2) demonstrated low basal activity (33). Elimination of these putative stems by a modest 5' deletion of only $23 \mathrm{nt}$ significantly increased fusion activity (33). A more precise genetic approach showed that a mutation in either the top or bottom strand of stem II resulted in a similar five-fold increase in translational activity (Fig. 2, G469C or C549G; 21). Compensatory mutations at these positions, predicted to maintain stem II 
pairing (Fig. 2, G469C and C549G), restored fusion activity to wild type levels (21). Mutations in either the top or bottom strands of stem III (Fig.2, G461C and C561G) increased rpoS translation although the compensatory mutations remained elevated in fusion activity compared to the wild type construct (21). The elevation in rpoS-lac activity in the presence of the individual stem mutations was comparable throughout the growth curve suggesting a similar role for the antisense element during exponential growth and SP.

\section{Regulatory RNAs and RpoS synthesis}

Genetic control mediated via small untranslated RNAs (sRNAs) is a rapidly expanding research area initially sparked by the anomaly of rpoS translation (54). This process is unique in that over expression of eight sRNAs are reported to influence RpoS translation, while no more than two sRNAs are reported to affect any other bacterial gene $(54,176)$. Two of the characterized sRNAs that affect RpoS translation, the thermoregulator DsrA and the membrane stress-induced RprA, are discussed in detail below. A list of the known sRNAs that influence RpoS translation are listed in Table 4 along with their inducing stimuli and affects on rpoS translation. 
Table 4. Regulatory RNA that effect rpoS translation in E. coli

\begin{tabular}{lllll}
\hline SRNA $^{\boldsymbol{a}}$ & $\begin{array}{c}\text { size } \\
\text { (nucleotides) }\end{array}$ & $\begin{array}{l}\text { Effect } \\
\text { on } \boldsymbol{r p o S}\end{array}$ & Stimuli & Reference \\
\hline DsrA & 85 & + & low temperature & $(158)$ \\
RprA & 105 & + & membrane stress & $(100)$ \\
OxyS & 109 & - & hydrogen peroxide & $(3)$ \\
RhyB & 90 & - & iron limitation & $(105)$ \\
RhyA & 45 & + & $?$ & $(176)$ \\
RyhB & 90 & + & $?$ & $(176)$ \\
RydB & 60 & - & $?$ & $(176)$ \\
RyeE & 86 & - & $?$ & $(176)$ \\
\hline
\end{tabular}

${ }^{a}$ The regulatory RNAs (sRNAs) that influence $r p o S$ translation are listed, along with their size and effect on rpoS translation. Stimuli that increase abundance of these sRNAs are also shown.

Additional evidence supporting the suggested structure of the rpoS leader region (Fig. 2) is the elegantly described interaction of the rpoS antisense element and non-translated regulatory RNA, DsrA $(54,91,101)$. DsrA is an 85 nt sRNA predicted to form a structure with three stemloops (157). Interestingly, two of the stem-loops activate different targets including rpoS translation and transcription of the capsular polysaccharide synthesis regulator, $\operatorname{RcsA}(157,158)$. The DsrA molecule contains $21 \mathrm{nt}$ of complementarity to the rpoS leader, including bases in the top strand of stems II and III (Fig. 2), and has been designated a thermoregulator of rpoS translation $(134,158)$. DsrA transcription is de-repressed at low temperatures $\left(\leq 30^{\circ} \mathrm{C}\right)$ and the transcript is also stabilized six-fold (134). DsrA regulates rpoS by intermolecular RNA interactions with the antisense element of the leader region, a process mediated by the RNAbinding protein $\mathrm{Hfq}(92,101)$. At low temperatures, rpoS translation increases in a DsrAdependent manner while at higher temperatures a mutation in $d s r A$ does not affect translational 
activity (158). The DsrA-rpoS interaction is thought to alter the secondary structure of the rpoS antisense element in a manner that promotes ribosome access and subsequent translation (101, $158,159)$.

Remarkably, another sRNA activates rpoS translation by targeting the same region of the rpoS leader as DsrA, stems II and III (Fig. 2; 100, 102). RprA is a $105 \mathrm{nt}$ structured RNA which exhibits non-contiguous sequence complementarity to the antisense element of the rpoS transcript. RprA was identified in E. coli as a multi-copy suppressor of decreased rpoS-lacZ activity in a $d s r A$ mutant background (100). In a wild type background, ectopic over expression of RprA increased rpoS-lacZ [pr] activity about six-fold (100).

Transcription of rprA is controlled by the phosphorelay system that regulates capsular polysaccharide synthesis genes, $\mathrm{R} \operatorname{csB}$ / $\mathrm{R} \operatorname{csC}$, in which $\mathrm{R} \operatorname{csC}$ is a transmembrane sensor and $\mathrm{RcsB}$ is the response regulator (102). A mutation in $r \operatorname{csB}$ eliminates the basal transcription of rprA while a mutation in $r c s C$ increases expression 10-fold (102). This relatively large increase in RprA was not enough to increase the activity of a rpoS translational fusion (102). However, a 50-fold increase in rprA transcription, caused by a constituitive $r c s C$ allele ( $r c s C$ 137), increased rpoS translation and RpoS abundance (102). A physiologically relevant role for activation of rpoS translation by RprA is awaiting description.

In addition to DsrA and RprA, at least six other sRNAs reportedly influence rpoS translation $(3,176)$. OxyS is a $109 \mathrm{nt}$ sRNA that activates and represses translation of numerous proteins, including RpoS (3). OxyS expression is stimulated by hydrogen peroxide although the 
mechanism by which it represses $r p o S$ translation is unknown (188). A genome wide search for additional regulatory RNAs in E. coli suggested two candidates that increased rpoS-lac translational activity, $r y h A$ and $r y b B$, when expressed from a multi-copy plasmid (176). Particular foldings of these RNA molecules show complementarity to rpoS stems II and III (Fig. 2). Three modest repressors of $r p o S$ translation were also suggested by this search $r y d B$, ryeE and the iron responsive $r y h B(105,176)$. All of these predicted sRNAs (except for rydB which was not tested) bind Hfq and require further investigations to confirm physiologically relevant roles in the regulation of RpoS synthesis (176).

\section{Hfq and RpoS synthesis}

Hfq (also referred to as HF-1) is a $11.2 \mathrm{kDa}$ protein and a pleiotropic regulator of diverse cellular functions. Hfq was first identified as a subunit of the RNA phage Q $\beta$ replicase (46) and subsequently found to associate with $30 \mathrm{~S}$ ribosomal subunits (39). Over the past 25 years, the known roles of Hfq have expanded to include activities as a RNA chaperone (108), a modulator of RNA sensitivity to cellular ribonucleases $(45,168,171)$, a stimulator of poly(A) tail elongation (58), and a post-transcriptional regulator of several RNAs including the rpoS transcript (20).

Hfq functions as a homo-hexameric ring (collectively termed Hfq) homologous to eukaryotic Sm proteins which are involved in pre-mRNA splicing and RNA degradation complexes $(109,143,146,190)$. Hfq has at least two RNA binding domains that prefer AU rich 
sequences, positioned close to structured regions $(19,171,190)$. It is thought that Hfq facilitates intermolecular RNA interactions through simultaneous binding of multiple transcripts (19).

Hfq mutants display a four to seven-fold reduction in RpoS synthesis during both exponential growth and SP, while SP induction of RpoS is not significantly altered (20). Coimmunoprecipitation studies using anti-Hfq recovered rpoS RNA from cell lysates and Hfq binds the rpoS leader region in vitro $(93,188)$. A $347 \mathrm{nt} 5$ ' deletion of the rpoS leader region abrogated Hfq control in vivo (33). In addition a point mutation within stem III also conferred Hfq independence (Fig. 2; 33). Together these results suggest that Hfq binds to an upstream region of the rpoS leader and contributes to basal translation. However, in conjunction with sRNAs, Hfq can also activate this process (21).

The exact mechanism by which Hfq influences rpoS synthesis is unknown. The nearly seven-fold reduction of rpoS translation in the $h f q$ mutant background cannot be rationalized by the loss of function of the known Hfq-dependent sRNAs. It is possible that Hfq binding destabilizes an inhibitory leader conformation or occludes a RNase E cleavage site. Other theories suggest that Hfq facilitates the interaction of unidentified activating sRNA(s) or it recruits ribosomes to the rpoS message. 


\section{H-NS and RpoS synthesis}

H-NS is a histone-like protein that primarily functions as a transcriptional repressor of $>100$ genes involved in environmental adaptation (63). As a homodimer H-NS binds specifically to curved regions of DNA, and is speculated to block transcription initiation at particular promoters (189). Several groups have demonstrated that H-NS also acts as a negative regulator of RpoS albeit by a post-transcriptional mechanism $(10,18,34,184)$. RpoS abundance is elevated in a hns mutant, an effect attributed to increased transcription and protein stabilization $(18,184)$. Despite the elevated level of RpoS in a hns mutant, SP induction of RpoS occurs normally (10).

\section{DksA and RpoS synthesis}

The DksA protein was first identified in E. coli as a multi-copy suppressor of the temperature sensitive growth and filamentation of a dnaK mutant (73). Since then, it has been implicated in a variety of cellular activities including cell division, the stringent response and Salmonella virulence $(11,22,177)$. In 1999, it was demonstrated that $d k s A$ mutants failed to accumulate RpoS during SP (177). Analysis of rpoS-lacZ fusions in a dksA mutant attributed the deficit mainly to defective translation (15-fold) with only a minor two-fold decrease in transcription (177). Four years later, DksA was reported as a critical factor for activation of $r p o S$ 
translation by ppGpp (22). The mechanism by which DksA influences RpoS translation is unknown and intriguing given the role for DksA in transcriptional modulation of RNAP through its secondary channel (117).

\section{RpoS stability}

Another regulatory pathway limiting RpoS abundance in growing cells is proteolytic degradation involving the ATP-dependent ClpXP protease and a response regulator called MviA (in S. enterica) or SprE / RssB in E. coli $(130,147)$. In this pathway MviA is activated by poorly characterized stimuli, including carbon starvation, through phosphorylation on D58, which substantially increases its ability to bind to RpoS. The relevant kinase has not yet been found $(34,61)$. The binding event (dependent on K173 of RpoS) results in a sequestered non-functional RpoS molecule and thus modulates RpoS activity in itself $(13,192)$. The MviA-RpoS complex also interacts with the ClpXP protease, which then actively degrades RpoS, recycling MviA (111, 130). RpoS elevates transcription of the response regulator during SP, thus constituting an auto-regulatory loop in which the concentration of MviA is a limiting factor for the rate of RpoS degradation in vivo $(132,142)$.

\section{Fis}

Fis is a transiently expressed DNA binding and bending protein involved in diverse cellular functions including transcriptional activation and repression of a large regulon (44). Additionally, Fis plays integral roles in site-specific DNA recombination, stimulation of excision / integration of $\lambda(6,7)$, DNA-replication $(43,51)$, and transposition (179). In general, Fis 
regulates cellular processes, including the expression of rRNA and tRNA, necessary for the transition to optimal growth, a time when Fis abundance peaks $(119,140)$.

The crystal structure of the 98 amino acid Fis protein displays $4 \alpha$-helical domains: A (residues 27-42), B (50-70), C (74-81), and D [85-94; (82)]. The N-terminal residues and both A helices of the homodimer are involved in stimulating site-specific DNA inversion (186). A truncated Fis protein, which is deleted for the N-terminal region, is defective in DNA inversion but maintains its ability to bind DNA and regulate gene expression as well as stimulate $\lambda$ excision (79). Helices $\mathrm{C}$ and $\mathrm{D}$ of each Fis monomer constitute helix-turn-helix (H-T-H) binding motifs that are required for recognition of the Fis consensus sequence ATTGNTCAAAATTTGANCANT $(60,186)$. Due to the spacing of these H-T-H elements, binding to opposite DNA strands is accompanied by bending of the DNA molecule. Fis-induced alterations in global topology, in accordance with fluctuating physiological conditions, indirectly influences transcriptional regulation (113).

\section{Regulation of Fis}

Fis is under strict genetic control and protein abundance fluctuates dramatically in response to growth environment. Fis levels peak (60,000 dimers / cell) 90 minutes after dilution into fresh medium and decline throughout exponential phase to very low levels, that remain low during SP $(2,8)$. Regulation of Fis occurs at the transcriptional level and is not mediated by changes in mRNA stability $(8,125,131)$. Activation of Fis transcription, which depends on the availability of particular nutrients (discussed below), occurs from a highly conserved promoter, 
$\mathrm{P}_{\text {fis. }}$ Increased Fis abundance results in a feedback loop in which Fis represses its own transcription (121). In E. coli and S. enterica, a single Fis binding site just upstream of $\mathrm{P}_{\text {fis }}$ is necessary for the majority of the six-fold transcriptional increase in a fis mutant background ( 8 , 174).

Activation of $\mathrm{P}_{\text {fis }}$ expression is dependent on the availability of the initiating nt of the fis transcript $(174,175)$. An investigation into growth phase regulation of $\mathrm{P}_{\text {fis }}$ revealed that a promoter sequence of $43 \mathrm{bp}$ was sufficient for Fis activation (174). Specifically, growth phase dependent regulation at $\mathrm{P}_{\text {fis }}$ was attributed to a less preferred initiating nt, CTP, of the fis transcript (174). At this position, nt replacement with either ATP or GTP, resulted in high levels of fis mRNA during early SP, a time when $\mathrm{P}_{\text {fis }}$ transcription is normally shut-off (174). The sensitivity of $\mathrm{P}_{\text {fis }}$ to the concentration of CTP was clearly demonstrated by a 20 -fold increase in activity upon the addition of excess CTP in vitro (175). It was also determined that the level of CTP in the growth medium directly correlates with $\mathrm{P}_{\text {fis }}$ expression (175). This form of transcriptional control is independent of $\operatorname{ppGpp}(8,174)$. However, the fis promoter is also subject to repression by the stringent response, an effect dependent upon seven consecutive G-C bp immediately preceding the transcriptional start site (121).

Nucleoprotein complexes involving the global regulators CRP, Fis and IHF also control $\mathrm{P}_{\text {fis }}$ activity. In a crp mutant background, fis mRNA levels are abnormally elevated during late exponential phase (114). The effect of CRP on $\mathrm{P}_{\text {fis }}$ transcription is not well understood and complicated by coordinate control with the Fis protein (114). In the absence of Fis, CRP activates $\mathrm{P}_{\text {fis }}$ while CRP in conjunction with Fis, synergistically represses activity (114). Another 
transiently expressed global transcription factor, IHF, whose abundance increases during exponential growth, is required for normal fis transcription (2). A mutation in $i h f$ decreases $\mathrm{P}_{\text {fis }}$ activity nearly four-fold by an unknown mechanism (131). The crosstalk between the global regulators CRP, Fis, and IHF at $\mathrm{P}_{\text {fis }}$ awaits further characterization. However, it is possible that alterations in DNA topology, induced by these regulators upon binding, indirectly influences $\mathrm{P}_{\text {fis }}$ activity (145).

\section{Functions of Fis}

\section{Inversion and Excision}

The Fis protein was first identified as a host factor required for site-specific DNA inversion $(72,78)$. The G-segment of phage Mu encodes tail fiber genes, the orientation of which determines host specificity, flanked by inverted repeats. During replicative transposition, phage with both orientations of the G-segment are produced by an inversion event dependent upon Fis and the phage-encoded invertase, Gin (72). Fis serves a similar function in the invertasome during flagellar phase variation in which binding of Fis to an enhancer element is necessary for Hin-mediated recombination $(72,96)$. Fis also plays a crucial role in the Cinmediated DNA inversion system of bacteriophage P1 (57).

The role of Fis was expanded to include partial regulation of the lysis versus lysogeny decision of phage $\lambda$ (166). DNA excision is stimulated 20 -fold in vitro by Fis binding to a 
region of $\lambda$ attP that overlaps a Xis binding site, a process that also requires the phage-encoded $\mathrm{X}$ is protein. In the absence of Xis, Fis induces $\lambda$ integration and lysogeny (7). Consistent with roles in chromosomal rearrangements, Fis also stimulates both Tn5 and IS50 transposition events in E. coli (179).

\section{Transcriptional regulation by Fis}

Over the last decade, Fis has been defined as a global regulator of transcription in both $S$. enterica and E. coli. Fis activates genes necessary for competitive replication, an effect that coincides with Fis abundance (Fis abundance peaks during initiation of exponential growth; 75). For instance, Fis binds several sites near the oriC promoter and is required for DNA replication of oriC minichromosomes $(43,51)$. Fis also strongly activates transcription of tRNA rRNA operons $(17,120)$. In the case of activation at $r r n B$ P1, the relatively short-lived open transcriptional complex is stabilized by Fis (191). In this process, Fis binds to a site centered upstream of the transcriptional start site and interacts with the $\alpha$ CTD of RNAP (1). A similar mechanism of activation occurs at the proP2 promoter and although at least four binding sites occur near this promoter only one, centered at -42 , is necessary for most of the effect (107). Recently, DNA microarrays comparing wild type and fis mutant backgrounds of S. enterica serovar Typhimurium demonstrated a major role for Fis in virulence gene expression, including type III secretion factors (75). This is consistent with a 100 -fold attenuation of virulence of a fis mutant in a mouse infection model (181). 
Fis also acts as a transcriptional repressor of several global regulators including gyrA, and gyrB, which code for subunits of DNA gyrase, a topoisomerase that induces negative DNA supercoiling $(74,144)$. In this process, Fis binds near the -10 hexamers of the gyrA and gyrB promoters and blocks transcription initiation (144). A mutation in fis increases gyrase activity and in general, Fis abundance inversely correlates with the concentrations of GyrA and GyrB (144). The influence of Fis on DNA topology both directly and indirectly, via DNA gyrase, control, probably regulates gene expression in accordance with cellular physiology.

\section{Regulation of Translational initiation}

Translational regulation is a common genetic strategy that allows the timely induction / repression of protein synthesis or maintains the appropriate constitutive levels. Some authors speculate that control at this level allows a quicker response to a particular stress because it

eliminates the time needed for transcription (61). In most cases, translational regulation depends on mRNA leader sequences either for sequence specific elements or structures that interact with trans-acting regulatory factors (protein or sRNAs; 16,54 ).

Translation is divided into three stages; initiation, elongation and termination. Translation initiation is the process by which ribosomal subunits recognize a particular region of a RNA molecule, referred to as the Shine-Dalgarno sequence (SD), followed by ribosome assembly preceding initial peptide bond formation (56). The primary contact is thought to be specific intermolecular RNA interactions between the mRNA and the 16S rRNA in conjunction with non-specific protein-RNA interactions (36). The ribosome initiation complex is composed 
of the ribosome, three initiating factors (IF1, IF2, and IF3), fMet-tRNA ${ }_{\mathrm{f}}^{\text {Met }}$, and of course, the template mRNA. Assembly of the ribosome initiation complex is reportedly stochastic (56). Subsequently, the ribosome moves along the RNA molecule and elongates the amino acid chain using residues of successive aminoacyl-tRNAs bound to the acceptor position of the ribosome via peptide-bonds. The polypeptide chain at the peptidyl site of the ribosome continues to grow until a stop codon is encountered and the polypeptide is released, followed by dissociation of the ribosomal subunits.

The composition, and at least partial function, of the ribosome changes as dividing cells enter SP. This transition is concomitant with a peak in the abundance of the ribosome modulation factor, RMF (173). RMF binds to the 50S subunit of the $70 \mathrm{~S}$ ribosome and mediates 70S-70S ribosomal dimerization $(172,173)$. The resulting $100 \mathrm{~S}$ dimer reportedly represents a storage form of the ribosome that is translationally inactive, possibly due to obstruction of the peptidyl-tRNA binding site (185). Upon subsequent culture in fresh medium, the 100S ribosomes dissociate back to the translationally active $70 \mathrm{~S}$ ribosomes by an uncharacterized process $(103,173)$. Another protein transiently associated with the ribosome is the SP-induced ribosome-associated, SRA (68). During SP, there is a RpoS-dependent increase in SRA abundance and the ratio of SRA bound specifically to the 30 S ribosomal subunit increases (68). The function of SRA is unknown. Due to the uncharacterized role of ribosomal composition in translational regulation, this review will focus specifically on the known factors that influence translation initiation: (i) the SD sequence and the spacer region between the SD sequence and the initiation codon, (ii) the initiation codon, (iii). RNA secondary structure and, (iv). the ribosomal protein $\mathrm{S} 1$. 


\section{Shine-Dalgarno sequence and Translational Regulation}

The SD sequence is a short stretch of nt preceding the coding sequence of most genes that indicates the site of translation initiation (153). In this process, the canonical SD sequence AGGA, or a variant, base pairs with the anti-SD sequence, UCCU, of the $3^{\prime}$ end of $16 \mathrm{~S}$ rRNA (a component of the $30 \mathrm{~S}$ ribosome along with 21 different proteins; 153). The free energy of this interaction reflects the efficiency of initiation; i.e. longer regions of complementarity are considered more attractive to ribosomes (97). For example, the SD sequence UAAGGAGG is four times more efficient in translation than AAGGA as measured using lacZ reporter fusions (136). However, it was also reported that extended SD / anti-SD interactions, beyond eight nt, actually decreased the activity of a reporter fusion possibly due to ribosome stalling at the clearance of the initiation stage (81). A study that examined the RBS of 124 mRNAs determined the average length of complementarity to be five nt and the minimum length was three nt. (161). Few reports have indicated that the presence of a SD sequence is not necessary for translation $(151,170,183)$. Another study demonstrated translation of an unleadered RNA, a process dependent upon a SD-like sequence downstream of the initiating codon (151).

Due to the size and correct positioning of the ribosome at the initiation codon, the spacer region between the SD sequence and the initiation codon also determines translational efficiency (52). In vivo experiments indicate that the efficacy of translation does not vary much when the spacer is between the range of five to $13 \mathrm{nt}$ and a spacing of nine $\mathrm{nt}$ is optimal (161). However, 
in these experiments other variables exist, including altered primary sequences and RNA secondary structures, which complicate result interpretation.

\section{Role of the initiation codon}

Translation initiation also involves base-pairing between the start codon and the anticodon of the initiator tRNA, fMet-tRNA $\mathrm{f}_{\mathrm{f}}^{\text {Met }}$. The preferred initiating codon, AUG, indicates the translational start of most translated RNAs, and is complementary to the anticodon of fMettRNA $_{\mathrm{f}}^{\text {Met }}$, UAC. Less frequently, GUG, UUG and CUG serve as start codons in which case weaker base pairing leads to less ternary complex (mRNA, the $30 \mathrm{~S}$ subunit and fMet-tRNA ${ }_{f}{ }^{\text {Met }}$ ) formation due to a decrease in overall complex stability (136). This is supported by in vivo analyses of reporter fusions that demonstrate decreased activity when GUG, UUG or CUG is substituted for AUG $(59,136,170)$. However, the decrease in activity compared to that of AUG containing reporters varied substantially among different initiation codons and is naturally exploited to control gene expression $(59,136)$. In general, the activity of initiation codons

follows the scheme: AUG $>$ GUG $>\operatorname{UUG}>\operatorname{CUG}(59,136)$. The second codon has also been shown to affect the rate of translation initiation and highly expressed genes often display GCU at this position $(40,136)$. 


\section{Structured Ribosome Binding Regions}

Translation initiation regions are often folded into complex secondary structures (e.g. rpoS; Fig. 2). An equilibrium exists such that at any given time a particular mRNA molecule may be partitioned between structured and non-structured conformations (37). This ratio is dependent upon the free energy of the different foldings (37). The theory that ribosomes are blocked by mRNA secondary structure predicts that stable foldings positively influence the folded:unfolded ratio and thus result in decreased initiation (37). This idea is at odds with a report suggesting that the $30 \mathrm{~S}$ ribosomal subunit recognizes a particular folded mRNA structure (66). Either way, the secondary structures of the ribosome-binding region (which can include far upstream and downstream interactions; Fig. 2) have been reported to both enhance and inhibit translation initiation (discussed below).

Phage T4 gene 38 encodes a protein required for long tail fiber assembly whose synthesis is enhanced by its ribosome-binding region (52). In this case, a $20 \mathrm{nt}$ stem-loop positions a distant SD sequence within five nt of the initiating codon facilitating translation initiation (52, 122). Mutations predicted to destabilize the structure resulted in decreased translation $(52,122)$. A similar mechanism of translational activation is reported for $\mathrm{T} 4$ gene $25(122,123)$.

Alternatively, structured regions that involve pairing of the SD sequence or the initiation codon result in decreased protein synthesis. Such is the case for the translation initiation region 
of the coat gene of the bacteriophage MS2 (37). The mRNA structure of this region pairs the SD sequence with nt downstream of the initiation codon (in this case AUG; 156). Mutations predicted to stabilize this inhibitory structure resulted in decreased protein expression, while disruption of base pairing at the SD sequence had the opposite effect (37). An important conclusion of that study is that a strict correlation exists between translation efficiency and the stability of the structured ribosome-binding region (37). Based on the effects of nt substitutions on both protein expression and predicted secondary structure stability it was also suggested that ribosomes bind only single-stranded regions of RNA molecules (37). This model contradicts the notion that local single-stranded regions, for example the SD sequence or the initiation codon, facilitates translation initiation (148). In these experiments it is difficult to attribute the changes in protein expression solely to changes in the folding energies considering that the primary sequence and the predicted RNA structures are also altered.

\section{Trans-acting Factors and Translational Regulation}

The ribosomal protein S1 (encoded by rpsA) is the largest ribosomal protein and essential for viability (162). S1 interacts with the head, platform and body of the $30 \mathrm{~S}$ subunit via intermolecular protein interactions late in assembly and its length is comparable to the longest dimension of the $30 \mathrm{~S}$ subunit (149). The N-terminal domain is responsible for protein-protein interactions while the $\mathrm{C}$-terminus contains four homologous repeats, the S1-motif, that constitute a RNA-binding domain $(26,162,163)$. S1 plays an essential role in translation using promiscuous RNA interactions (with a preference for polyU, polyA and polyC nt sequences) to 
promote binding of the ribosome to mRNA $(16,138,160,162,163)$. Ribosomal associated S1mRNA interactions occur anywhere from 22-145 nt upstream of the initiation codon, and purified S1 binds RNA in vitro $(14-16,135,150)$.

Reports of actual translational regulation by S1 are scarce and the best described system is autogenous repression $(14,15,141)$. Despite the absence of a canonical SD sequence, rpsA is actively translated and S1 repression occurs at this level (155). The proposed model relies on S1-mediated recognition and disruption of a phylogenetically conserved secondary structure of the rpsA mRNA leader ( -90 to +20 with respect to the first nt of the initiation codon) that forms a non-contiguous SD sequence (15).

A recent report demonstrates that $\mathrm{S} 1$ binds to another leader region, the rpoS message in Pseudomonas aeruginosa (150). This interaction occurs specifically during exponential phase and depends on $78 \mathrm{nt}$ upstream of the initiation codon however, the translational consequence of S1 binding to rpoS was not determined (150). These results suggest a possible role in regulation of rpoS translation, although the model is counterintuitive; $\mathrm{S} 1$ recruits ribosomes to the rpoS message during a time when translation is minimal.

\section{Literature Cited}

1. Aiyar, S. E., S. M. McLeod, W. Ross, C. A. Hirvonen, M. S. Thomas, R. C. Johnson, and R. L. Gourse. 2002. Architecture of Fis-activated transcription complexes at the Escherichia coli rrnB P1 and rrnE P1 promoters. J Mol Biol 316:501-16. 
2. Ali Azam, T., A. Iwata, A. Nishimura, S. Ueda, and A. Ishihama. 1999. Growth phase-dependent variation in protein composition of the Escherichia coli nucleoid. $\mathrm{J}$ Bacteriol 181:6361-70.

3. Altuvia, S., D. Weinstein-Fischer, A. Zhang, L. Postow, and G. Storz. 1997. A small, stable RNA induced by oxidative stress: role as a pleiotropic regulator and antimutator. Cell 90:43-53.

4. Angerer, A., S. Enz, M. Ochs, and V. Braun. 1995. Transcriptional regulation of ferric citrate transport in Escherichia coli K-12. Fecl belongs to a new subfamily of sigma 70type factors that respond to extracytoplasmic stimuli. Mol Microbiol 18:163-74.

5. Artsimovitch, I., V. Patlan, S. Sekine, M. N. Vassylyeva, T. Hosaka, K. Ochi, S. Yokoyama, and D. G. Vassylyev. 2004. Structural basis for transcription regulation by alarmone ppGpp. Cell 117:299-310.

6. Ball, C. A., and R. C. Johnson. 1991. Efficient excision of phage lambda from the Escherichia coli chromosome requires the Fis protein. J Bacteriol 173:4027-31.

7. Ball, C. A., and R. C. Johnson. 1991. Multiple effects of Fis on integration and the control of lysogeny in phage lambda. J Bacteriol 173:4032-8.

8. Ball, C. A., R. Osuna, K. C. Ferguson, and R. C. Johnson. 1992. Dramatic changes in Fis levels upon nutrient upshift in Escherichia coli. J Bacteriol 174:8043-56.

9. Barker, M. M., T. Gaal, and R. L. Gourse. 2001. Mechanism of regulation of transcription initiation by ppGpp. II. Models for positive control based on properties of RNAP mutants and competition for RNAP. J Mol Biol 305:689-702.

10. Barth, M., C. Marschall, A. Muffler, D. Fischer, and R. Hengge-Aronis. 1995. Role for the histone-like protein H-NS in growth phase-dependent and osmotic regulation of sigma S and many sigma S-dependent genes in Escherichia coli. J Bacteriol 177:345564.

11. Bass, S., Q. Gu, and A. Christen. 1996. Multicopy suppressors of pre mutant Escherichia coli include two HtrA (DegP) protease homologs (HhoAB), DksA, and a truncated R1pA. J Bacteriol 178:1154-61. 
12. Bearson, S. M., W. H. Benjamin, Jr., W. E. Swords, and J. W. Foster. 1996. Acid shock induction of RpoS is mediated by the mouse virulence gene mviA of Salmonella typhimurium. J Bacteriol 178:2572-9.

13. Becker, G., E. Klauck, and R. Hengge-Aronis. 2000. The response regulator RssB, a recognition factor for sigmaS proteolysis in Escherichia coli, can act like an anti-sigmaS factor. Mol Microbiol 35:657-66.

14. Boni, I. V., V. S. Artamonova, and M. Dreyfus. 2000. The last RNA-binding repeat of the Escherichia coli ribosomal protein S1 is specifically involved in autogenous control. J Bacteriol 182:5872-9.

15. Boni, I. V., V. S. Artamonova, N. V. Tzareva, and M. Dreyfus. 2001. Non-canonical mechanism for translational control in bacteria: synthesis of ribosomal protein S1. Embo J 20:4222-32.

16. Boni, I. V., and A. M. Borodin. 1990. [Rare initiation codons are regulators of expression of the rpoC gene]. Bioorg Khim 16:1134-7.

17. Bosch, L., L. Nilsson, E. Vijgenboom, and H. Verbeek. 1990. FIS-dependent transactivation of tRNA and rRNA operons of Escherichia coli. Biochim Biophys Acta 1050:293-301.

18. Brescia, C. C., M. K. Kaw, and D. D. Sledjeski. 2004. The DNA binding protein H-NS binds to and alters the stability of RNA in vitro and in vivo. J Mol Biol 339:505-14.

19. Brescia, C. C., P. J. Mikulecky, A. L. Feig, and D. D. Sledjeski. 2003. Identification of the Hfq-binding site on DsrA RNA: Hfq binds without altering DsrA secondary structure. Rna 9:33-43.

20. Brown, L., and T. Elliott. 1996. Efficient translation of the RpoS sigma factor in Salmonella typhimurium requires host factor I, an RNA-binding protein encoded by the hfq gene. J Bacteriol 178:3763-70.

21. Brown, L., and T. Elliott. 1997. Mutations that increase expression of the rpoS gene and decrease its dependence on $h f q$ function in Salmonella typhimurium. J Bacteriol 179:65662.

22. Brown, L., D. Gentry, T. Elliott, and M. Cashel. 2002. DksA affects ppGpp induction of RpoS at a translational level. J Bacteriol 184:4455-65. 
23. Burgess, R. R., A. A. Travers, J. J. Dunn, and E. K. Bautz. 1969. Factor stimulating transcription by RNA polymerase. Nature 221:43-6.

24. Busby, S., and R. H. Ebright. 1999. Transcription activation by catabolite activator protein (CAP). J Mol Biol 293:199-213.

25. Butukhanov, V. D., M. A. Bobyk, V. Tsyrenov, and I. S. Kulaev. 1979. [Possible role of high molecular weight polyphosphates in ATP synthesis from exogenous adenine by the culture of Corynebacterium sp., strain VSTI-301]. Biokhimiia 44:1321-8.

26. Bycroft, M., T. J. Hubbard, M. Proctor, S. M. Freund, and A. G. Murzin. 1997. The solution structure of the S1 RNA binding domain: a member of an ancient nucleic acidbinding fold. Cell 88:235-42.

27. Cashel, M. 1969. The control of ribonucleic acid synthesis in Escherichia coli. IV. Relevance of unusual phosphorylated compounds from amino acid-starved stringent strains. J Biol Chem 244:3133-41.

28. Castuma, C. E., R. Huang, A. Kornberg, and R. N. Reusch. 1995. Inorganic polyphosphates in the acquisition of competence in Escherichia coli. J Biol Chem 270:12980-3.

29. Cayley, S., B. A. Lewis, H. J. Guttman, and M. T. Record, Jr. 1991. Characterization of the cytoplasm of Escherichia coli K-12 as a function of external osmolarity. Implications for protein-DNA interactions in vivo. J Mol Biol 222:281-300.

30. Chen, C. Y., N. A. Buchmeier, S. Libby, F. C. Fang, M. Krause, and D. G. Guiney. 1995. Central regulatory role for the RpoS sigma factor in expression of Salmonella dublin plasmid virulence genes. J Bacteriol 177:5303-9.

31. Chen, C. Y., L. Eckmann, S. J. Libby, F. C. Fang, S. Okamoto, M. F. Kagnoff, J. Fierer, and D. G. Guiney. 1996. Expression of Salmonella typhimurium rpoS and rpoSdependent genes in the intracellular environment of eukaryotic cells. Infect Immun 64:4739-43.

32. Colland, F., M. Barth, R. Hengge-Aronis, and A. Kolb. 2000. sigma factor selectivity of Escherichia coli RNA polymerase: role for CRP, IHF and Lrp transcription factors. Embo J 19:3028-37. 
33. Cunning, C., L. Brown, and T. Elliott. 1998. Promoter substitution and deletion analysis of upstream region required for $r p o S$ translational regulation. J Bacteriol 180:4564-70.

34. Cunning, C., and T. Elliott. 1999. RpoS synthesis is growth rate regulated in Salmonella typhimurium, but its turnover is not dependent on acetyl phosphate synthesis or PTS function. J Bacteriol 181:4853-62.

35. de Crombrugghe, B., S. Busby, and H. Buc. 1984. Cyclic AMP receptor protein: role in transcription activation. Science 224:831-8.

36. de Smit, M. 1998. RNA Structure and Function. Cold Spring Harbor Laboratory Press, Cold Spring Harbor.

37. de Smit, M. H., and J. van Duin. 1990. Secondary structure of the ribosome binding site determines translational efficiency: a quantitative analysis. Proc Natl Acad Sci U S A 87:7668-72.

38. Ding, Q., S. Kusano, M. Villarejo, and A. Ishihama. 1995. Promoter selectivity control of Escherichia coli RNA polymerase by ionic strength: differential recognition of osmoregulated promoters by E sigma D and E sigma S holoenzymes. Mol Microbiol 16:649-56.

39. DuBow, M. S., T. Ryan, R. A. Young, and T. Blumenthal. 1977. Host factor for coliphage Q beta RNA replication: presence in procaryotes and association with the $30 \mathrm{~S}$ ribosomal subunit in Escherichia coli. Mol Gen Genet 153:39-43.

40. Dunn, J. J., and F. W. Studier. 1983. Complete nucleotide sequence of bacteriophage T7 DNA and the locations of T7 genetic elements. J Mol Biol 166:477-535.

41. Fang, F. C., S. J. Libby, N. A. Buchmeier, P. C. Loewen, J. Switala, J. Harwood, and D. G. Guiney. 1992. The alternative sigma factor katF (rpoS) regulates Salmonella virulence. Proc Natl Acad Sci U S A 89:11978-82.

42. Farewell, A., K. Kvint, and T. Nystrom. 1998. Negative regulation by RpoS: a case of sigma factor competition. Mol Microbiol 29:1039-51.

43. Filutowicz, M., W. Ross, J. Wild, and R. L. Gourse. 1992. Involvement of Fis protein in replication of the Escherichia coli chromosome. J Bacteriol 174:398-407. 
44. Finkel, S. E., and R. C. Johnson. 1992. The Fis protein: it's not just for DNA inversion anymore. Mol Microbiol 6:3257-65.

45. Folichon, M., V. Arluison, O. Pellegrini, E. Huntzinger, P. Regnier, and E. Hajnsdorf. 2003. The poly(A) binding protein Hfq protects RNA from RNase E and exoribonucleolytic degradation. Nucleic Acids Res 31:7302-10.

46. Franze de Fernandez, M. T., W. S. Hayward, and J. T. August. 1972. Bacterial proteins required for replication of phage Q ribonucleic acid. Pruification and properties of host factor I, a ribonucleic acid-binding protein. J Biol Chem 247:824-31.

47. Fu, J. C., L. Ding, and S. Clarke. 1991. Purification, gene cloning, and sequence analysis of an L-isoaspartyl protein carboxyl methyltransferase from Escherichia coli. J Biol Chem 266:14562-72.

48. Gaal, T., W. Ross, S. T. Estrem, L. H. Nguyen, R. R. Burgess, and R. L. Gourse. 2001. Promoter recognition and discrimination by EsigmaS RNA polymerase. Mol Microbiol 42:939-54.

49. Gentry, D. R., and M. Cashel. 1995. Cellular localization of the Escherichia coli SpoT protein. J Bacteriol 177:3890-3.

50. Gentry, D. R., V. J. Hernandez, L. H. Nguyen, D. B. Jensen, and M. Cashel. 1993. Synthesis of the stationary-phase sigma factor sigma s is positively regulated by ppGpp. $\mathrm{J}$ Bacteriol 175:7982-9.

51. Gille, H., J. B. Egan, A. Roth, and W. Messer. 1991. The FIS protein binds and bends the origin of chromosomal DNA replication, oriC, of Escherichia coli. Nucleic Acids Res 19:4167-72.

52. Gold, L., D. Pribnow, T. Schneider, S. Shinedling, B. S. Singer, and G. Stormo. 1981. Translational initiation in prokaryotes. Annu Rev Microbiol 35:365-403.

53. Gosset, G., Z. Zhang, S. Nayyar, W. A. Cuevas, and M. H. Saier, Jr. 2004. Transcriptome analysis of Crp-dependent catabolite control of gene expression in Escherichia coli. J Bacteriol 186:3516-24.

54. Gottesman, S. 2004. The small RNA regulators of Escherichia coli: roles and mechanisms*. Annu Rev Microbiol 58:303-28. 
55. Gralla, J. D. 2005. Escherichia coli ribosomal RNA transcription: regulatory roles for ppGpp, NTPs, architectural proteins and a polymerase-binding protein. Mol Microbiol 55:973-7.

56. Gualerzi, C. O., and C. L. Pon. 1990. Initiation of mRNA translation in prokaryotes. Biochemistry 29:5881-9.

57. Haffter, P., and T. A. Bickle. 1987. Purification and DNA-binding properties of FIS and Cin, two proteins required for the bacteriophage P1 site-specific recombination system, cin. J Mol Biol 198:579-87.

58. Hajnsdorf, E., and P. Regnier. 2000. Host factor Hfq of Escherichia coli stimulates elongation of poly(A) tails by poly(A) polymerase I. Proc Natl Acad Sci U S A 97:15015.

59. Hartz, D., D. S. McPheeters, L. Green, and L. Gold. 1991. Detection of Escherichia coli ribosome binding at translation initiation sites in the absence of tRNA. J Mol Biol 218:99-105.

60. Hengen, P. N., S. L. Bartram, L. E. Stewart, and T. D. Schneider. 1997. Information analysis of Fis binding sites. Nucleic Acids Res 25:4994-5002.

61. Hengge-Aronis, R. 2002. Signal transduction and regulatory mechanisms involved in control of the sigma(S) (RpoS) subunit of RNA polymerase. Microbiol Mol Biol Rev 66:373-95.

62. Hengge-Aronis, R., W. Klein, R. Lange, M. Rimmele, and W. Boos. 1991. Trehalose synthesis genes are controlled by the putative sigma factor encoded by rpoS and are involved in stationary-phase thermotolerance in Escherichia coli. J Bacteriol 173:791824.

63. Hommais, F., E. Krin, C. Laurent-Winter, O. Soutourina, A. Malpertuy, J. P. Le Caer, A. Danchin, and P. Bertin. 2001. Large-scale monitoring of pleiotropic regulation of gene expression by the prokaryotic nucleoid-associated protein, H-NS. Mol Microbiol 40:20-36.

64. Huisman, G. W., and R. Kolter. 1994. Sensing starvation: a homoserine lactone-dependent signaling pathway in Escherichia coli. Science 265:537-9. 
65. Ichikawa, J. K., C. Li, J. Fu, and S. Clarke. 1994. A gene at 59 minutes on the Escherichia coli chromosome encodes a lipoprotein with unusual amino acid repeat sequences. J Bacteriol 176:1630-8.

66. Iserentant, D., and W. Fiers. 1980. Secondary structure of mRNA and efficiency of translation initiation. Gene 9:1-12.

67. Ishihama, A. 2000. Functional modulation of Escherichia coli RNA polymerase. Annu Rev Microbiol 54:499-518.

68. Izutsu, K., C. Wada, Y. Komine, T. Sako, C. Ueguchi, S. Nakura, and A. Wada. 2001. Escherichia coli ribosome-associated protein SRA, whose copy number increases during stationary phase. J Bacteriol 183:2765-73.

69. Jishage, M., and A. Ishihama. 1995. Regulation of RNA polymerase sigma subunit synthesis in Escherichia coli: intracellular levels of sigma 70 and sigma 38. J Bacteriol 177:6832-5.

70. Jishage, M., A. Iwata, S. Ueda, and A. Ishihama. 1996. Regulation of RNA polymerase sigma subunit synthesis in Escherichia coli: intracellular levels of four species of sigma subunit under various growth conditions. J Bacteriol 178:5447-51.

71. Jishage, M., K. Kvint, V. Shingler, and T. Nystrom. 2002. Regulation of sigma factor competition by the alarmone ppGpp. Genes Dev 16:1260-70.

72. Johnson, R. C., Bruist, M. F., Simon, R. I. 1986. Host protein requirements for in vitro site specific DNA inversion. Cell 46:531-539.

73. Kang, P. J., and E. A. Craig. 1990. Identification and characterization of a new Escherichia coli gene that is a dosage-dependent suppressor of a dnaK deletion mutation. J Bacteriol 172:2055-64.

74. Keane, O. M., and C. J. Dorman. 2003. The gyr genes of Salmonella enterica serovar Typhimurium are repressed by the factor for inversion stimulation, Fis. Mol Genet Genomics 270:56-65.

75. Kelly, A., M. D. Goldberg, R. K. Carroll, V. Danino, J. C. Hinton, and C. J. Dorman. 2004. A global role for Fis in the transcriptional control of metabolism and type III secretion in Salmonella enterica serovar Typhimurium. Microbiology 150:2037-53. 
76. Kim, E. Y., M. S. Shin, J. H. Rhee, and H. E. Choy. 2004. Factors influencing preferential utilization of RNA polymerase containing sigma-38 in stationary-phase gene expression in Escherichia coli. J Microbiol 42:103-10.

77. Kim, K. S., N. N. Rao, C. D. Fraley, and A. Kornberg. 2002. Inorganic polyphosphate is essential for long-term survival and virulence factors in Shigella and Salmonella spp. Proc Natl Acad Sci U S A 99:7675-80.

78. Koch, C., and R. Kahmann. 1986. Purification and properties of the Escherichia coli host factor required for inversion of the $\mathrm{G}$ segment in bacteriophage $\mathrm{Mu}$. J Biol Chem 261:15673-8.

79. Koch, C., O. Ninnemann, H. Fuss, and R. Kahmann. 1991. The N-terminal part of the E.coli DNA binding protein FIS is essential for stimulating site-specific DNA inversion but is not required for specific DNA binding. Nucleic Acids Res 19:5915-22.

80. Kolb, A., S. Busby, H. Buc, S. Garges, and S. Adhya. 1993. Transcriptional regulation by cAMP and its receptor protein. Annu Rev Biochem 62:749-95.

81. Komarova, A. V., L. S. Tchufistova, E. V. Supina, and I. V. Boni. 2002. Protein S1 counteracts the inhibitory effect of the extended Shine-Dalgarno sequence on translation. Rna 8:1137-47.

82. Kostrewa, D., J. Granzin, D. Stock, H. W. Choe, J. Labahn, and W. Saenger. 1992. Crystal structure of the factor for inversion stimulation FIS at 2.0 A resolution. J Mol Biol 226:209-26.

83. Kundu, T. K., S. Kusano, and A. Ishihama. 1997. Promoter selectivity of Escherichia coli RNA polymerase sigmaF holoenzyme involved in transcription of flagellar and chemotaxis genes. J Bacteriol 179:4264-9.

84. Kuroda, A., H. Murphy, M. Cashel, and A. Kornberg. 1997. Guanosine tetra- and pentaphosphate promote accumulation of inorganic polyphosphate in Escherichia coli. J Biol Chem 272:21240-3.

85. Kusano, S., Q. Ding, N. Fujita, and A. Ishihama. 1996. Promoter selectivity of Escherichia coli RNA polymerase E sigma 70 and E sigma 38 holoenzymes. Effect of DNA supercoiling. J Biol Chem 271:1998-2004. 
86. Kusano, S., and A. Ishihama. 1997. Stimulatory effect of trehalose on formation and activity of Escherichia coli RNA polymerase E sigma38 holoenzyme. J Bacteriol 179:3649-54.

87. Lange, R., D. Fischer, and R. Hengge-Aronis. 1995. Identification of transcriptional start sites and the role of ppGpp in the expression of $r p o S$, the structural gene for the sigma S subunit of RNA polymerase in Escherichia coli. J Bacteriol 177:4676-80.

88. Lange, R., and R. Hengge-Aronis. 1994. The cellular concentration of the sigma S subunit of RNA polymerase in Escherichia coli is controlled at the levels of transcription, translation, and protein stability. Genes Dev 8:1600-12.

89. Lange, R., and R. Hengge-Aronis. 1991. Growth phase-regulated expression of bolA and morphology of stationary-phase Escherichia coli cells are controlled by the novel sigma factor sigma S. J Bacteriol 173:4474-81.

90. Lange, R., and R. Hengge-Aronis. 1991. Identification of a central regulator of stationary-phase gene expression in Escherichia coli. Mol Microbiol 5:49-59.

91. Lease, R. A., and M. Belfort. 2000. A trans-acting RNA as a control switch in Escherichia coli: DsrA modulates function by forming alternative structures. Proc Natl Acad Sci U S A 97:9919-24.

92. Lease, R. A., M. E. Cusick, and M. Belfort. 1998. Riboregulation in Escherichia coli: DsrA RNA acts by RNA:RNA interactions at multiple loci. Proc Natl Acad Sci U S A 95:12456-61.

93. Lease, R. A., and S. A. Woodson. 2004. Cycling of the Sm-like protein Hfq on the DsrA small regulatory RNA. J Mol Biol 344:1211-23.

94. Lee, J. Y., J. E. Kwak, J. Moon, S. H. Eom, E. C. Liong, J. D. Pedelacq, J. Berendzen, and S. W. Suh. 2001. Crystal structure and functional analysis of the SurE protein identify a novel phosphatase family. Nat Struct Biol 8:789-94.

95. Li, C., P. Y. Wu, and M. Hsieh. 1997. Growth-phase-dependent transcriptional regulation of the pcm and surE genes required for stationary-phase survival of Escherichia coli. Microbiology 143 ( Pt 11):3513-20.

96. Lim, H. M., H. J. Lee, C. Jaxel, and M. Nadal. 1997. Hin-mediated inversion on positively supercoiled DNA. J Biol Chem 272:18434-9. 
97. Lithwick, G., and H. Margalit. 2003. Hierarchy of sequence-dependent features associated with prokaryotic translation. Genome Res 13:2665-73.

98. Maeda, H., N. Fujita, and A. Ishihama. 2000. Competition among seven Escherichia coli sigma subunits: relative binding affinities to the core RNA polymerase. Nucleic Acids Res 28:3497-503.

99. Maeda, H., M. Jishage, T. Nomura, N. Fujita, and A. Ishihama. 2000. Two extracytoplasmic function sigma subunits, sigma(E) and sigma(FecI), of Escherichia coli: promoter selectivity and intracellular levels. J Bacteriol 182:1181-4.

100. Majdalani, N., S. Chen, J. Murrow, K. St John, and S. Gottesman. 2001. Regulation of RpoS by a novel small RNA: the characterization of RprA. Mol Microbiol 39:138294.

101. Majdalani, N., C. Cunning, D. Sledjeski, T. Elliott, and S. Gottesman. 1998. DsrA RNA regulates translation of RpoS message by an anti-antisense mechanism, independent of its action as an antisilencer of transcription. Proc Natl Acad Sci U S A 95:12462-7.

102. Majdalani, N., D. Hernandez, and S. Gottesman. 2002. Regulation and mode of action of the second small RNA activator of RpoS translation, RprA. Mol Microbiol 46:813-26.

103. Maki, Y., H. Yoshida, and A. Wada. 2000. Two proteins, YfiA and YhbH, associated with resting ribosomes in stationary phase Escherichia coli. Genes Cells 5:965-74.

104. Makinoshima, H., S. Aizawa, H. Hayashi, T. Miki, A. Nishimura, and A. Ishihama. 2003. Growth phase-coupled alterations in cell structure and function of Escherichia coli. J Bacteriol 185:1338-45.

105. Masse, E., and S. Gottesman. 2002. A small RNA regulates the expression of genes involved in iron metabolism in Escherichia coli. Proc Natl Acad Sci U S A 99:4620-5.

106. McCann, M. P., J. P. Kidwell, and A. Matin. 1991. The putative sigma factor KatF has a central role in development of starvation-mediated general resistance in Escherichia coli. J Bacteriol 173:4188-94.

107. McLeod, S. M., S. E. Aiyar, R. L. Gourse, and R. C. Johnson. 2002. The C-terminal domains of the RNA polymerase alpha subunits: contact site with Fis and localization 
during co-activation with CRP at the Escherichia coli proP P2 promoter. J Mol Biol 316:517-29.

108. Moll, I., T. Afonyushkin, O. Vytvytska, V. R. Kaberdin, and U. Blasi. 2003.

Coincident Hfq binding and RNase E cleavage sites on mRNA and small regulatory RNAs. Rna 9:1308-14.

109. Moller, T., T. Franch, P. Hojrup, D. R. Keene, H. P. Bachinger, R. G. Brennan, and P. Valentin-Hansen. 2002. Hfq: a bacterial Sm-like protein that mediates RNA-RNA interaction. Mol Cell 9:23-30.

110. Muffler, A., M. Barth, C. Marschall, and R. Hengge-Aronis. 1997. Heat shock regulation of sigmaS turnover: a role for DnaK and relationship between stress responses mediated by sigmaS and sigma32 in Escherichia coli. J Bacteriol 179:445-52.

111. Muffler, A., D. Fischer, S. Altuvia, G. Storz, and R. Hengge-Aronis. 1996. The response regulator RssB controls stability of the sigma(S) subunit of RNA polymerase in Escherichia coli. Embo J 15:1333-9.

112. Mulvey, M. R., and P. C. Loewen. 1989. Nucleotide sequence of katF of Escherichia coli suggests KatF protein is a novel sigma transcription factor. Nucleic Acids Res 17:9979-91.

113. Muskhelishvili, G., and A. Travers. 2003. Transcription factor as a topological homeostat. Front Biosci 8:d279-85.

114. Nasser, W., R. Schneider, A. Travers, and G. Muskhelishvili. 2001. CRP modulates fis transcription by alternate formation of activating and repressing nucleoprotein complexes. J Biol Chem 276:17878-86.

115. Neidhardt, F. C. 1996. Escherichia coli and Salmonella, second ed, vol. 1. ASM Press, Washington, DC.

116. Nickels, B. E., S. J. Garrity, V. Mekler, L. Minakhin, K. Severinov, R. H. Ebright, and A. Hochschild. 2005. The interaction between sigma70 and the beta-flap of Escherichia coli RNA polymerase inhibits extension of nascent RNA during early elongation. Proc Natl Acad Sci U S A 102:4488-93.

117. Nickels, B. E., and A. Hochschild. 2004. Regulation of RNA polymerase through the secondary channel. Cell 118:281-4. 
118. Nickerson, C. A., and R. Curtiss, 3rd. 1997. Role of sigma factor RpoS in initial stages of Salmonella typhimurium infection. Infect Immun 65:1814-23.

119. Nilsson, L., and V. Emilsson. 1994. Factor for inversion stimulation-dependent growth rate regulation of individual tRNA species in Escherichia coli. J Biol Chem 269:9460-5.

120. Nilsson, L., A. Vanet, E. Vijgenboom, and L. Bosch. 1990. The role of FIS in trans activation of stable RNA operons of E. coli. Embo J 9:727-34.

121. Ninnemann, O., C. Koch, and R. Kahmann. 1992. The E.coli fis promoter is subject to stringent control and autoregulation. Embo J 11:1075-83.

122. Nivinskas, R., N. Malys, V. Klausa, R. Vaiskunaite, and E. Gineikiene. 1999. Posttranscriptional control of bacteriophage $\mathrm{T} 4$ gene 25 expression: mRNA secondary structure that enhances translational initiation. J Mol Biol 288:291-304.

123. Nivinskas, R., R. Vaiskunaite, and A. Raudonikiene. 1993. Expression of bacteriophage T4 gene 25 is regulated via RNA secondary structure in the translational initiation region. J Mol Biol 230:717-21.

124. Nystrom, T. 2004. Growth versus maintenance: a trade-off dictated by RNA polymerase availability and sigma factor competition? Mol Microbiol 54:855-62.

125. Osuna, R., D. Lienau, K. T. Hughes, and R. C. Johnson. 1995. Sequence, regulation, and functions of fis in Salmonella typhimurium. J Bacteriol 177:2021-32.

126. Paesold, G., and M. Krause. 1999. Analysis of rpoS mRNA in Salmonella dublin: identification of multiple transcripts with growth-phase-dependent variation in transcript stability. J Bacteriol 181:1264-8.

127. Paul, B. J., W. Ross, T. Gaal, and R. L. Gourse. 2004. rRNA transcription in Escherichia coli. Annu Rev Genet 38:749-70.

128. Perederina, A., V. Svetlov, M. N. Vassylyeva, T. H. Tahirov, S. Yokoyama, I. Artsimovitch, and D. G. Vassylyev. 2004. Regulation through the secondary channel-structural framework for ppGpp-DksA synergism during transcription. Cell 118:297-309.

129. Phillips, N. F., P. J. Horn, and H. G. Wood. 1993. The polyphosphate- and ATPdependent glucokinase from Propionibacterium shermanii: both activities are catalyzed by the same protein. Arch Biochem Biophys 300:309-19. 
130. Pratt, L. A., and T. J. Silhavy. 1996. The response regulator SprE controls the stability of RpoS. Proc Natl Acad Sci U S A 93:2488-92.

131. Pratt, T. S., T. Steiner, L. S. Feldman, K. A. Walker, and R. Osuna. 1997. Deletion analysis of the fis promoter region in Escherichia coli: antagonistic effects of integration host factor and Fis. J Bacteriol 179:6367-77.

132. Pruteanu, M., and R. Hengge-Aronis. 2002. The cellular level of the recognition factor RssB is rate-limiting for sigmaS proteolysis: implications for RssB regulation and signal transduction in sigmaS turnover in Escherichia coli. Mol Microbiol 45:1701-13.

133. Rao, N. N., and A. Kornberg. 1996. Inorganic polyphosphate supports resistance and survival of stationary-phase Escherichia coli. J Bacteriol 178:1394-400.

134. Repoila, F., and S. Gottesman. 2001. Signal transduction cascade for regulation of RpoS: temperature regulation of DsrA. J Bacteriol 183:4012-23.

135. Ringquist, S., T. Jones, E. E. Snyder, T. Gibson, I. Boni, and L. Gold. 1995. Highaffinity RNA ligands to Escherichia coli ribosomes and ribosomal protein S1: comparison of natural and unnatural binding sites. Biochemistry 34:3640-8.

136. Ringquist, S., S. Shinedling, D. Barrick, L. Green, J. Binkley, G. D. Stormo, and L. Gold. 1992. Translation initiation in Escherichia coli: sequences within the ribosomebinding site. Mol Microbiol 6:1219-29.

137. Robbe-Saule, V., C. Coynault, and F. Norel. 1995. The live oral typhoid vaccine Ty21a is a $r p o S$ mutant and is susceptible to various environmental stresses. FEMS Microbiol Lett 126:171-6.

138. Roberts, M. W., and J. C. Rabinowitz. 1989. The effect of Escherichia coli ribosomal protein S1 on the translational specificity of bacterial ribosomes. J Biol Chem 264:222835 .

139. Rojiani, M. V., H. Jakubowski, and E. Goldman. 1989. Effect of variation of charged and uncharged tRNA(Trp) levels on ppGpp synthesis in Escherichia coli. J Bacteriol 171:6493-502.

140. Ross, W., J. F. Thompson, J. T. Newlands, and R. L. Gourse. 1990. E.coli Fis protein activates ribosomal RNA transcription in vitro and in vivo. Embo J 9:3733-42. 
141. Ruckman, J., S. Ringquist, E. Brody, and L. Gold. 1994. The bacteriophage T4 regB ribonuclease. Stimulation of the purified enzyme by ribosomal protein S1. J Biol Chem 269:26655-62.

142. Ruiz, N., C. N. Peterson, and T. J. Silhavy. 2001. RpoS-dependent transcriptional control of sprE: regulatory feedback loop. J Bacteriol 183:5974-81.

143. Sauter, C., J. Basquin, and D. Suck. 2003. Sm-like proteins in Eubacteria: the crystal structure of the Hfq protein from Escherichia coli. Nucleic Acids Res 31:4091-8.

144. Schneider, R., A. Travers, T. Kutateladze, and G. Muskhelishvili. 1999. A DNA architectural protein couples cellular physiology and DNA topology in Escherichia coli. Mol Microbiol 34:953-64.

145. Schneider, R., A. Travers, and G. Muskhelishvili. 2000. The expression of the Escherichia coli fis gene is strongly dependent on the superhelical density of DNA. Mol Microbiol 38:167-75.

146. Schumacher, M. A., R. F. Pearson, T. Moller, P. Valentin-Hansen, and R. G. Brennan. 2002. Structures of the pleiotropic translational regulator Hfq and an Hfq-RNA complex: a bacterial Sm-like protein. Embo J 21:3546-56.

147. Schweder, T., K. H. Lee, O. Lomovskaya, and A. Matin. 1996. Regulation of Escherichia coli starvation sigma factor (sigma s) by ClpXP protease. J Bacteriol 178:470-6.

148. Selker, E., and C. Yanofsky. 1979. Nucleotide sequence of the trpC-trpB intercistronic region from Salmonella typhimurium. J Mol Biol 130:135-43.

149. Sengupta, J., R. K. Agrawal, and J. Frank. 2001. Visualization of protein S1 within the 30S ribosomal subunit and its interaction with messenger RNA. Proc Natl Acad Sci U S A 98:11991-6.

150. Sevo, M., E. Buratti, and V. Venturi. 2004. Ribosomal protein S1 specifically binds to the 5' untranslated region of the Pseudomonas aeruginosa stationary-phase sigma factor rpoS mRNA in the logarithmic phase of growth. J Bacteriol 186:4903-9.

151. Shean, C. S., and M. E. Gottesman. 1992. Translation of the prophage lambda cl transcript. Cell 70:513-22. 
152. Shiba, T., K. Tsutsumi, H. Yano, Y. Ihara, A. Kameda, K. Tanaka, H. Takahashi, M. Munekata, N. N. Rao, and A. Kornberg. 1997. Inorganic polyphosphate and the induction of rpoS expression. Proc Natl Acad Sci U S A 94:11210-5.

153. Shine, J., and L. Dalgarno. 1974. The 3'-terminal sequence of Escherichia coli 16S ribosomal RNA: complementarity to nonsense triplets and ribosome binding sites. Proc Natl Acad Sci U S A 71:1342-6.

154. Sitnikov, D. M., J. B. Schineller, and T. O. Baldwin. 1996. Control of cell division in Escherichia coli: regulation of transcription of ftsQA involves both rpoS and SdiAmediated autoinduction. Proc Natl Acad Sci U S A 93:336-41.

155. Skouv, J., J. Schnier, M. D. Rasmussen, A. R. Subramanian, and S. Pedersen. 1990. Ribosomal protein $\mathrm{S} 1$ of Escherichia coli is the effector for the regulation of its own synthesis. J Biol Chem 265:17044-9.

156. Skripkin, E. A., M. R. Adhin, M. H. de Smit, and J. van Duin. 1990. Secondary structure of the central region of bacteriophage MS2 RNA. Conservation and biological significance. J Mol Biol 211:447-63.

157. Sledjeski, D., and S. Gottesman. 1995. A small RNA acts as an antisilencer of the HNS-silenced rcsA gene of Escherichia coli. Proc Natl Acad Sci U S A 92:2003-7.

158. Sledjeski, D. D., A. Gupta, and S. Gottesman. 1996. The small RNA, DsrA, is essential for the low temperature expression of RpoS during exponential growth in Escherichia coli. Embo J 15:3993-4000.

159. Sledjeski, D. D., C. Whitman, and A. Zhang. 2001. Hfq is necessary for regulation by the untranslated RNA DsrA. J Bacteriol 183:1997-2005.

160. Sorensen, M. A., J. Fricke, and S. Pedersen. 1998. Ribosomal protein S1 is required for translation of most, if not all, natural mRNAs in Escherichia coli in vivo. J Mol Biol 280:561-9.

161. Stormo, G. D., T. D. Schneider, and L. M. Gold. 1982. Characterization of translational initiation sites in E. coli. Nucleic Acids Res 10:2971-96.

162. Subramanian, A. R. 1983. Structure and functions of ribosomal protein S1. Prog Nucleic Acid Res Mol Biol 28:101-42. 
163. Suryanarayana, T., and A. R. Subramanian. 1984. Function of the repeating homologous sequences in nucleic acid binding domain of ribosomal protein $\mathrm{S} 1$. Biochemistry 23:1047-51.

164. Takayanagi, Y., K. Tanaka, and H. Takahashi. 1994. Structure of the 5' upstream region and the regulation of the rpoS gene of Escherichia coli. Mol Gen Genet 243:52531.

165. Tanaka, K., Y. Takayanagi, N. Fujita, A. Ishihama, and H. Takahashi. 1993. Heterogeneity of the principal sigma factor in Escherichia coli: the rpoS gene product, sigma 38 , is a second principal sigma factor of RNA polymerase in stationary-phase Escherichia coli. Proc Natl Acad Sci U S A 90:3511-5.

166. Thompson, J. F., L. Moitoso de Vargas, C. Koch, R. Kahmann, and A. Landy. 1987. Cellular factors couple recombination with growth phase: characterization of a new component in the lambda site-specific recombination pathway. Cell 50:901-8.

167. Touati, E., E. Dassa, J. Dassa, P. L. Boquet, and D. Touati. 1991. Are appR and katF the same Escherichia coli gene encoding a new sigma transcription initiation factor? Res Microbiol 142:29-36.

168. Tsui, H. C., G. Feng, and M. E. Winkler. 1997. Negative regulation of mutS and mutH repair gene expression by the Hfq and RpoS global regulators of Escherichia coli K-12. J Bacteriol 179:7476-87.

169. Ueguchi, C., N. Misonou, and T. Mizuno. 2001. Negative control of rpoS expression by phosphoenolpyruvate: carbohydrate phosphotransferase system in Escherichia coli. J Bacteriol 183:520-7.

170. VanEtten, H., S. Jorgensen, J. Enkerli, and S. F. Covert. 1998. Inducing the loss of conditionally dispensable chromosomes in Nectria haematococca during vegetative growth. Curr Genet 33:299-303.

171. Vytvytska, O., J. S. Jakobsen, G. Balcunaite, J. S. Andersen, M. Baccarini, and A. von Gabain. 1998. Host factor I, Hfq, binds to Escherichia coli ompA mRNA in a growth rate-dependent fashion and regulates its stability. Proc Natl Acad Sci U S A 95:14118-23. 
172. Wada, A., R. Mikkola, C. G. Kurland, and A. Ishihama. 2000. Growth phase-coupled changes of the ribosome profile in natural isolates and laboratory strains of Escherichia coli. J Bacteriol 182:2893-9.

173. Wada, A., Y. Yamazaki, N. Fujita, and A. Ishihama. 1990. Structure and probable genetic location of a "ribosome modulation factor" associated with $100 \mathrm{~S}$ ribosomes in stationary-phase Escherichia coli cells. Proc Natl Acad Sci U S A 87:2657-61.

174. Walker, K. A., C. L. Atkins, and R. Osuna. 1999. Functional determinants of the Escherichia coli fis promoter: roles of $-35,-10$, and transcription initiation regions in the response to stringent control and growth phase-dependent regulation. J Bacteriol 181:1269-80.

175. Walker, K. A., P. Mallik, T. S. Pratt, and R. Osuna. 2004. The Escherichia coli Fis promoter is regulated by changes in the levels of its transcription initiation nucleotide CTP. J Biol Chem 279:50818-28.

176. Wassarman, K. M., F. Repoila, C. Rosenow, G. Storz, and S. Gottesman. 2001. Identification of novel small RNAs using comparative genomics and microarrays. Genes Dev 15:1637-51.

177. Webb, C., M. Moreno, M. Wilmes-Riesenberg, R. Curtiss, 3rd, and J. W. Foster. 1999. Effects of DksA and ClpP protease on sigma S production and virulence in Salmonella typhimurium. Mol Microbiol 34:112-23.

178. Weber, H., T. Polen, J. Heuveling, V. F. Wendisch, and R. Hengge. 2005. Genomewide analysis of the general stress response network in Escherichia coli: sigmaSdependent genes, promoters, and sigma factor selectivity. J Bacteriol 187:1591-603.

179. Weinreich, M. D., and W. S. Reznikoff. 1992. Fis plays a role in Tn5 and IS50 transposition. J Bacteriol 174:4530-7.

180. Wilmes-Riesenberg, M. R., J. W. Foster, and R. Curtiss, 3rd. 1997. An altered rpoS allele contributes to the avirulence of Salmonella typhimurium LT2. Infect Immun 65:203-10.

181. Wilson, R. L., S. J. Libby, A. M. Freet, J. D. Boddicker, T. F. Fahlen, and B. D. Jones. 2001. Fis, a DNA nucleoid-associated protein, is involved in Salmonella typhimurium SPI-1 invasion gene expression. Mol Microbiol 39:79-88. 
182. Wood, H. G., and J. E. Clark. 1988. Biological aspects of inorganic polyphosphates. Annu Rev Biochem 57:235-60.

183. Wu, C. J., and G. R. Janssen. 1996. Translation of vph mRNA in Streptomyces lividans and Escherichia coli after removal of the 5' untranslated leader. Mol Microbiol 22:33955.

184. Yamashino, T., C. Ueguchi, and T. Mizuno. 1995. Quantitative control of the stationary phase-specific sigma factor, sigma S, in Escherichia coli: involvement of the nucleoid protein H-NS. Embo J 14:594-602.

185. Yoshida, H., Y. Maki, H. Kato, H. Fujisawa, K. Izutsu, C. Wada, and A. Wada. 2002. The ribosome modulation factor (RMF) binding site on the $100 \mathrm{~S}$ ribosome of Escherichia coli. J Biochem (Tokyo) 132:983-9.

186. Yuan, H. S., S. E. Finkel, J. A. Feng, M. Kaczor-Grzeskowiak, R. C. Johnson, and R. E. Dickerson. 1991. The molecular structure of wild-type and a mutant Fis protein: relationship between mutational changes and recombinational enhancer function or DNA binding. Proc Natl Acad Sci U S A 88:9558-62.

187. Zgurskaya, H. I., M. Keyhan, and A. Matin. 1997. The sigma S level in starving Escherichia coli cells increases solely as a result of its increased stability, despite decreased synthesis. Mol Microbiol 24:643-51.

188. Zhang, A., S. Altuvia, A. Tiwari, L. Argaman, R. Hengge-Aronis, and G. Storz. 1998. The OxyS regulatory RNA represses rpoS translation and binds the Hfq (HF-I) protein. Embo J 17:6061-8.

189. Zhang, A., S. Rimsky, M. E. Reaban, H. Buc, and M. Belfort. 1996. Escherichia coli protein analogs StpA and H-NS: regulatory loops, similar and disparate effects on nucleic acid dynamics. Embo J 15:1340-9.

190. Zhang, A., K. M. Wassarman, J. Ortega, A. C. Steven, and G. Storz. 2002. The Smlike Hfq protein increases OxyS RNA interaction with target mRNAs. Mol Cell 9:11-22.

191. Zhi, H., X. Wang, J. E. Cabrera, R. C. Johnson, and D. J. Jin. 2003. Fis stabilizes the interaction between RNA polymerase and the ribosomal promoter $r r n B \mathrm{P} 1$, leading to transcriptional activation. J Biol Chem 278:47340-9. 
192. Zhou, Y., and S. Gottesman. 1998. Regulation of proteolysis of the stationary-phase sigma factor RpoS. J Bacteriol 180:1154-8.

193. Zuker, M. 2003. Mfold web server for nucleic acid folding and hybridization prediction. Nucleic Acids Res 31:3406-15. 


\title{
Role of ppGpp in rpoS Stationary Phase Regulation in Escherichia coli
}

\author{
Matthew Hirsch and Thomas Elliott ${ }^{*}$
}

Department of Microbiology, Immunology and Cell Biology, West Virginia University Health Sciences Center, Morgantown, WV 26506

* corresponding author: telliott@hsc.wvu.edu

P.O. Box 9177

Morgantown, WV 26506

(304) 293-2676

FAX (304) 293-7823

Abbreviation: ppGpp, guanosine 5'-diphosphate, 3'-diphosphate 


\section{Abstract}

The bacterial sigma factor RpoS is strongly induced under a variety of stress conditions and during growth into stationary phase. Here, we use rpoS-lac fusions in E. coli to investigate control acting at the level of RpoS synthesis, which is especially evident when cells approach stationary phase in rich medium. Previous work has shown that the small molecule ppGpp is required for normal levels of RpoS in stationary phase. Despite the attraction of a model in which the ppGpp level controls stationary phase induction of RpoS, careful measurement of rpoS-lac expression in a mutant lacking ppGpp shows similar effects during both exponential growth and stationary phase: the main effect of ppGpp is on basal expression. In addition, a modest regulatory defect was associated with the mutant lacking ppGpp, delaying the time at which full expression is achieved by 2 to 3 hours. Deletion analysis showed that the defect in basal expression was distributed over several sequence elements, while the regulatory defect mapped to the region upstream of the rpoS ribosome-binding site (RBS) that contains a cis-acting antisense element. A number of other genes that have been suggested as regulators of $r p o S$ were tested, including $d k s A, d s r A$, barA, ppkx, and $h f q$. With the exception of the dksA mutant, which had a modest defect in Luria-Bertani medium, none of these mutants was defective for rpoS stationaryphase induction. Even a short rpoS segment starting at 24 nt upstream of the AUG initiation codon was sufficient to confer substantial stationary phase regulation, which was mainly posttranscriptional. The effect of RBS-proximal sequence was independent of all known trans-acting factors, including ppGpp. 


\section{Introduction}

The rpoS gene encodes a sigma factor, $\sigma^{\mathrm{S}}$ or RpoS, which is required for expression of a large number of genes in response to various stresses, including nutrient limitation and osmotic challenge, and during growth into stationary phase (see references 15 and 21 for reviews). The rpoS gene has been found in a variety of Gram-negative bacteria and its function and regulation have been studied extensively in the enteric species Escherichia coli and Salmonella enterica serovar Typhimurium (here referred to as S. enterica). RpoS is also a virulence factor for S. enterica (13) and its expression is induced when these bacteria enter mammalian host cells (9). It is not clear how information about stress, nutrient limitation and host environment is used to control RpoS. Increased RpoS abundance has been reported to be regulated at many levels including transcription initiation and elongation $(17,18,30,34)$, translation $(19,22,24)$, and protein stability $(19,31,35)$. RpoS protein activity is also regulated (32). No in vitro system that mimics any aspect of in vivo control of RpoS synthesis has been described.

Genetic analysis has led to the idea that some, perhaps most, regulation of RpoS synthesis occurs at the posttranscriptional level via an inhibitory mRNA secondary structure $(7,20,23)$. An upstream antisense element has been localized through computer analysis of RNA folding and identification of compensatory mutations (7, our unpublished data); the antisense element can pair with the ribosomebinding site (RBS) region and inhibit rpoS translation. This proposed RNA structure is not yet supported by physical evidence. It is, however, strongly supported by genetic analysis of the DsrA RNA, a small untranslated RNA which acts as an anti-antisense RNA, increasing rpoS expression (23). DsrA RNA is important for expression of $r p o S$ in E. coli at growth temperatures at or below $30^{\circ} \mathrm{C}$ 
$(33,39)$, but is not required in S. enterica (unpublished data). It is not yet clear whether the antisense element functions in other regulatory inputs to RpoS.

Mutations in more than 20 genes have been identified as affecting RpoS synthesis alone. Many of these "regulators" exhibit highly pleiotropic phenotypes, and it seems unlikely that most act directly on rpoS expression. Often, such mutants show changes in the shape of the growth curve even in rich medium. Thus, their effects on RpoS may be a secondary consequence of altered growth rates and early or prolonged entry into stationary phase. There are clearly strong selective forces both for RpoS activity (in early stationary phase) and against it (in both late stationary and exponential phase). Given these forces, it is more than a formal possibility that uncharacterized strain differences may influence the observed regulation. Known examples include the wild type S. enterica strain LT2, which is defective in the RpoS protein turnover mechanism 3,11); and the widely-used E. coli strain MC4100, which is a relA mutant, and is often used despite the reported role for ppGpp in RpoS regulation (14). Thus, even more than for most regulatory systems, the results observed may depend on which strain was used and how the cells were grown.

Here, we investigate the induction of RpoS that occurs in the wild type E. coli strain MG1655 as cells are grown to stationary phase in LB medium, usually at $37^{\circ} \mathrm{C}$. This medium was chosen because the induction ratio (stationary phase expression / exponential phase expression) is particularly high under these conditions: ca. 35-fold as measured using an rpoS-lac protein fusion. Previous work showed that RpoS abundance is greatly reduced in a MG1655 $\Delta$ relA $\Delta$ spoT mutant which lacks ppGpp (14). (This genetic background is referred to below as ppGpp ${ }^{\mathrm{o}}$ for convenience). Artificially increasing 
ppGpp levels by synthesis of a truncated RelA protein also leads to a very large and rapid increase in RpoS abundance, while substantially increased RpoS abundance can also be observed in certain spoT mutants that have modestly elevated ppGpp (14).

Another study concluded that the main effect of ppGpp is on transcription elongation across the rpoS leader (17). This conclusion was based on apparently normal stationary phase and ppGppregulation of plasmid-borne rpoS-lac fusions (to codon 23 of rpoS) which had been deleted for the known rpoS promoters. However, the source of this low-level residual transcription was not identified. There is also an apparent conflict between this conclusion and experiments using ppGpp overproduction (8) which found that rpoS mRNA abundance is not elevated by ppGpp overproduction during exponential phase, pointing to translation control of rpoS by ppGpp.

To further investigate these questions, we employed a set of lacUV5 promoter substitution and deletion derivatives of rpoS-lac, which allow sensitive, quantitative measurement of rpoS expression in LB medium in different mutant backgrounds.

\section{Materials and Methods.}

Bacterial strains and construction. Strains used in this study for physiological experiments are derived from the wild type E. coli K-12 strain MG1655. The parental strain was CF7968, which is MG1655 that has been corrected to $\operatorname{rph}^{+}(16)$ and deleted for lacIZ, obtained from M. Cashel. This lac deletion extends between MluI sites in lacI and lacZ and was constructed by D. Vinella. Many of the 
lac fusions used in this work have been described previously $(6,7,10)$. These fusions are placed in the E. coli trp operon as described (12). Phage P1 vir was used for transduction; P1 growth and transduction were carried out by standard methods (37).

Media and growth conditions. Bacteria were grown at $37^{\circ} \mathrm{C}$ (with one exception as noted below) in Luria-Bertani (LB) medium (37) and on nutrient agar plates containing $5 \mathrm{~g}$ of $\mathrm{NaCl}$ per liter, except where indicated. Minimal agar was prepared with NCE medium containing $0.2 \%$ glucose (4). Antibiotics were added to final concentrations in selective plates as follows: $20 \mu \mathrm{g}$ of tetracycline hydrochloride $/ \mathrm{ml}$ (10 $\mu \mathrm{g} / \mathrm{ml}$ for minimal medium), $20 \mu \mathrm{g}$ of chloramphenicol / $\mathrm{ml}, 50 \mu \mathrm{g}$ of kanamycin sulfate / $\mathrm{ml}$ (except for $h f q$ crosses, as noted below) and $30 \mu \mathrm{g}$ of sodium ampicillin / $\mathrm{ml}(100 \mu \mathrm{g} / \mathrm{ml}$ when selecting for plasmids).

New mutations affecting putative trans-acting factors. New insertion mutations were made by the method of Yu et al (43) employing host strain DY330 (E. coli W3110 AlacU169 gal490 [ $\Delta$ cI857ts $\Delta($ cro-bioA) $]$. Primers containing $20 \mathrm{nt}$ of tet homology at the $3^{\prime}$ end were used to amplify the tetAR genes from plasmid pWM7 (25). PCR was performed using Taq polymerase (Qiagen) as suggested by the manufacturer. Amplified DNA was purified by a QIAquik PCR purification kit (Qiagen); residual template DNA was then removed by digestion with DpnI, which cuts specifically at methylated GATC sites, followed by repurification of the PCR product and elution in a volume equal to the original PCR reaction. Heat-induction and transformation of DY330 was as described (43) using 5 $\mu 1$ of DNA; transformants were selected at $30^{\circ} \mathrm{C}$. 
Primers for tet amplification were as follows (tet homology in upper case):

$d k s A$ atgcaagaagggcaaaaccgtaaaacatcgtccetgagtattctcgecatCTCTTGGGTTATCAAGAGGG, ttagccagccatctgtttttcgcgaatttcagccagcgttttgcagtcgaACTCGACATCTTGGTTACCG;

barA ctttctcaatttaacagtgtgacettaattgtcccataacgCTCTTGGGTTATCAAGAGGG, ccagcgtcataaaaagccgattgctactcgacaagacatccattaACTCGACATCTTGGTTACCG;

ppkx ggtcaggaaaagctatacatcgaaaaagagctcagttggtCTCTTGGGTTATCAAGAGGG, tcgtcggeccgcaaagtattaagcggegatttctggtgtaACTCGACATCTTGGTTACCG.

The resulting deletion/insertion mutations result in loss of target gene sequence as follows: $d k s A$ (codons 18 through 136); barA (7 bp upstream of codon 1 through the termination codon); ppkx (codon 15 of ppk through codon 507 of ppx). Strains were checked for the insertion/deletion by PCR with flanking primers. Sequences of these primers are available on request. Only a small number of candidate insertions was checked for each gene knockout experiment; in every case, a PCR product of the predicted size was observed.

The same general method was used to substitute the bla $\left(\mathrm{Amp}^{\mathrm{R}}\right)$ gene for $\mathrm{Kan}^{\mathrm{R}}$ of certain lac fusions. The primers used have bla homology at their 3' ends (upper case) and the template was pBR322:

tcatgaacaataaaactgtctgcttacataaacagtaataTGAAGACGAAAGGGCCTCGTGATAC, gcgtaatgctctgccagtgttacaaccaattaaccaattcTTACCAATGCTTAATCAGTGAGGCAC.

In contrast to the transformations used to construct tet insertions, for the bla substitutions it was found that only a minority of $A m p^{R}$ transformants had lost the $\operatorname{Kan}^{\mathrm{R}}$ marker as predicted for events of 
the desired type (the frequency of the correct event ranged from 1-25\%). However, the $A m p^{R}$ marker of the desired class $\left(\mathrm{Kan}^{\mathrm{S}}\right)$ showed $100 \%$ linkage to $\mathrm{Lac}^{+}$upon backcross.

Other mutations affecting trans-acting factors. The relA deletion used was from strain CF3032 (ArelA252::kan argA::Tn10; 26). Since most of the lac fusions used here are marked with

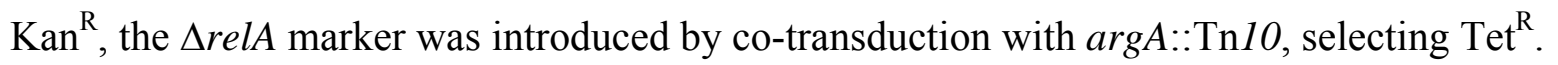
Transductants were screened for the Rel- phenotype by testing sensitivity to SMGL (serine, methionine, glycine and leucine) on minimal glucose plates with tetracycline. When comparing wild type with $\operatorname{ppGpp}^{\circ}\left(\Delta\right.$ relA $\Delta$ spoT) strains, the $\operatorname{relA}{ }^{+} \operatorname{spoT}^{+}$control strains also carry the $\arg A:: \operatorname{Tn} 10$ (with one exception noted below). The spoT deletion used was from strain CF1693 ( $\Delta$ relA251::kan AspoT207::cat; 42). The dsrA deletion used was from strain DDS724 ( $\Delta d s r A 5$ with linked Tn10), obtained from D. Sledjeski. This deletion is described in reference 39. The dsrA deletion was introduced by co-transduction with the linked Tn10. For comparison with $\Delta d s r A$ strains, wild type $d s r A^{+}$strains also carry this linked Tn10. To construct strains for the epistasis test of $d s r A$ and relA spoT, the $\Delta$ relA252::kan marker from CF3032 was introduced by linkage to $\operatorname{argA::Tn10}$ and then the Tn10 was removed by subsequent transduction to $\mathrm{Arg}^{+}$.

The $h f q$ insertion used was from strain TX2822 ( $h f q-1:: \Omega-\mathrm{Km})$, obtained from M. Winkler (40). This insertion is at codon 41 of the 102 codon $h f q$ gene. We encountered difficulty using $\operatorname{Kan}^{\mathrm{R}}$ to select for transfer of $h f q-1:: \Omega-\mathrm{Km}$. In fact, all the strains we obtained carrying $h f q-1:: \Omega-\mathrm{Km}$ grew very poorly when streaked out on either LB or NB agar containing $50 \mu \mathrm{g} / \mathrm{ml}$ kanamycin, showing a typical pattern 
of colonies in the streak (suggesting suppression). This behavior is not understood. Growth was normal in the absence of kanamycin. To construct the needed strains, selection for transductants carrying $h f q-$ 1:: $\Omega-\mathrm{Km}$ was carried out on LB agar with kanamycin at $25 \mu \mathrm{g} / \mathrm{ml}$ at room temperature, and transductants were then purified on LB agar without kanamycin at $37^{\circ} \mathrm{C}$. Successful introduction of the hfq insertion (and all other deletions) was confirmed by PCR.

rpoS-lac fusion. We have previously described the detailed method used to make the rpoS-lac constructs employed for most of the experiments in the present work $(6,10)$. They use the general system originally designed by Simons et al. (38), as modified (12). The relevant gene segments include (in order) an upstream $\mathrm{Kan}^{\mathrm{r}}$ element, tandem transcriptional terminators, and the promoter or regulatory sequence under investigation, followed by the lac operon. This assembly is placed in single copy in the bacterial chromosome (at trp); therefore all strains carry a wild-copy of rpoS. Most lac fusions used in this study have lacZ placed to form either an operon or protein fusion at the EagI site at codon 73 of rpoS. A different set of fusions, to codon 8 of $r p o S$, was used for the last set of experiments as described below.

Some constructs carried the native rpoS promoter (6). In the others, including the KpnI construct as well as the numbered deletions, rpoS-lac is expressed from the lacUV5 promoter (lacUV5p) with a constant lac-derived leader of $36 \mathrm{nt}$ plus several restriction sites, followed by different amounts of $r p o S$ sequence; these constructs vary only in the extent of the deletion that removes rpoS sequence from the upstream side. The lacUV5p is derived from pRS476 (38); it includes only one of the cyclic AMP receptor protein (CRP) half-sites on the upstream side and the lac operator on the downstream side. 
The reference $K p n I$ site construct (construct $\mathrm{K}$ ), as well as the $\Delta 1$ and $\Delta 2$ constructs, were all previously described (10). The $\Delta 3$ and $\Delta 4$ deletions were made by PCR in exactly the same way as $\Delta 1$ and $\triangle 2$; the PCR amplified segments were re-sequenced to ensure that no unwanted changes had been introduced. These deletion endpoints for $\Delta 2$ through $\Delta 4$ are illustrated with respect to the sequence in Fig. 1. All deletion endpoints are numbered starting from the first transcribed nucleotide for transcripts initiated from the $r p o S$ promoter. We have taken this transcript sequence to begin with GGGUGAACAG (the first $\mathrm{G}$ is $\mathrm{nt} 1 ; 17$ ). The coordinates of the first base pair that is still present in each construct are as follows: construct $\mathrm{K}$, nt $73 ; \Delta 1$, nt $344 ; \Delta 2$, nt $454 ; \Delta 3 \mathrm{nt} 477 ; \Delta 4 \mathrm{nt} 541$. The rpoS ATG initiation codon is at nt 565.

Another construct in which lacUV5 drives rpoS expression starting from "+1" of rpoS (nt 1) was constructed by PCR on a rpoS-lac template with a lac-specific oligonucleotide together with the following oligonucleotide (the lacUV5 mutation in the promoter's -10 region is shown in bold; $r p o S$ homology is shown by italics):

CGCGAATTCAGGCTTTACACTTTATGCTTCCGGCTCGTATAATGTGTGGAATTGGGTGAACAGAGTGCTAACAAAA TG.

Transcripts originating from lacUV5p in this construct are predicted to contain the 5' sequence AAUUGGGUGAACAGAGTGCTAACAAAATG, where the underlined nucleotides are derived from rpoS sequence. This construct does not include the lac operator. The PCR product was substituted as an EcoRI-KpnI fragment in several steps, and this segment was sequenced to make sure that no unwanted mutations had been introduced by the PCR step. The strain with this fusion carried in the bacterial chromosome is TE8378. 
Second method for making promoter fusions. We subsequently developed a convenient method for making constructs in which rpoS (or any gene) can be expressed from lacUV5p, by employing the lambda lysogenic strain background and technique of Yu et al (43). The general transformation method is the same as described in the section above on tet insertions.

For this purpose, we first placed tetAR upstream of lacUV5p, replacing $\operatorname{Kan}^{\mathrm{R}}$ (38) in the standard $\Delta 1$ fusion by using the following two oligonucleotides:

ATAAAGTGTAAAGCCTGGGGTGCCTAATGAGTGAGAATTCACTCGACATCTTGGTTACCG (tetR homology in italic, lacUV5p homology elsewhere) and TCATGAACAATAAAACTGTCTGCTTACATAAACAGTAATACTCTTGGGTTATCAAGAGGG (tetA homology in italic, kan homology elewhere):

In the second step, the marked lacUV5 promoter was joined to each of the desired target sites by PCR amplifying ther tetAR-lacUV5p segment with an oligonucleotide which included appropriate rpoS homology attached 5' to the lacUV5-specific sequence: AATTCCACACATTAATACGAG. As in the first step, on the upstream side the oligonucleotide for PCR was chosen so that the tetAR-lacUV5p substitution will replace the kan gene of the standard fusion. The resulting constructs are marked with Tet $^{\mathrm{r}}$, join to lacUV5p directly to the desired target sequence, and do not include the lac operator. With this method, we made a new set of deletions extending to various positions directly upstream of the rpoS ATG initiation codon as described above in the text. Depending on the strain used for lambda redmediated transcformation, the resulting constructs are lac [op] or [pr] fusions at codon 8 of rpoS. The full lacUV5p sequence is GAATTCTCACTCATTAGGCACCCCAGGCTTTACACTTTATGCTTCCGGCTCGTATAATGTGTGGAATT. 
Constructs made by this method were confirmed genetically as $\mathrm{Kan}^{\mathrm{s}}$ and physically by PCR with primers specific to tetR and lac, followed by DNA sequencing across the lacUV5p and the first 200 to $300 \mathrm{nt}$ at the joint to rpoS. After verification, the fusion constructs were transduced into the CF7968 background by selecting Tet ${ }^{\mathrm{r}}$.

The lac fusions marked with $\mathrm{Kan}^{\mathrm{r}}$, as designed by Simons et al. (38), carry tandem insertions of a terminator between the drug resistance cassette and the site where test segments are joined to lac; terminators were not explicitly included in the fusions marked with $\operatorname{Tet}^{\mathrm{r}}$ made by the new method. This was considered unlikely to be necessary because of the weak activity of the tetR promoter and its more than 20-fold dependence on tetracycline for induction. Indeed, assays of constructs grown in the presence or absence of tetracycline showed that induced transcription from tetR accounts for $\approx 10 \%$ of lac transcription from exponential phase cells; the contribution from uninduced transcription (i.e. our standard growth condition) is therefore negligible.

Assay of $\beta$-galactosidase. Cells were centrifuged and resuspended in Z-buffer (100 mM $\mathrm{NaPO}_{4}, \mathrm{pH}$ 7.0, $\left.10 \mathrm{mM} \mathrm{KCl}, 1 \mathrm{mM} \mathrm{MgSO} 4\right)$, then permeabilized by treatment with SDS and chloroform (27). The samples from exponential phase time points were concentrated before assay, to be approximately equal in density to samples from later times. Assays were performed in Z-buffer containing $50 \mathrm{mM} \beta$-mercaptoethanol by a kinetic method using a plate reader (Molecular Dynamics). Activities ( $\mathrm{OD}_{420}$ per min) are normalized to actual cell density $\left(\mathrm{OD}_{650}\right)$ and were always compared to appropriate controls assayed at the same time. All $\beta$-galactosidase assays were performed within $3 \mathrm{hr}$ of the time of sampling. The values shown are averages of at least three experiments with standard 
deviations of less than $20 \%$, except for the very low values from exponential phase for the ppGpp ${ }^{\mathrm{O}}$

mutant, where the standard deviations were less than $30 \%$. But we should point out that comparisons of the relative defect in stationary phase induction involve ratios of experimental values, with a corresponding increase in the uncertainty.

Figure 3. Partial Restriction map of the $n l p D$ and rpoS open reading frames.

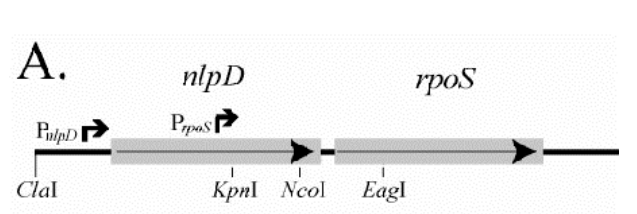

- Panel A shows a partial restriction map of the DNA encompassing the $n l p D$ and $r p o S$ open reading frames of E. coli, which consist of 321 and 330 codons, respectively. The function of $n l p D$ (encoding a lipoprotein) is not related to that of rpoS. Arrows indicate the orientation of the two ORFs and the known

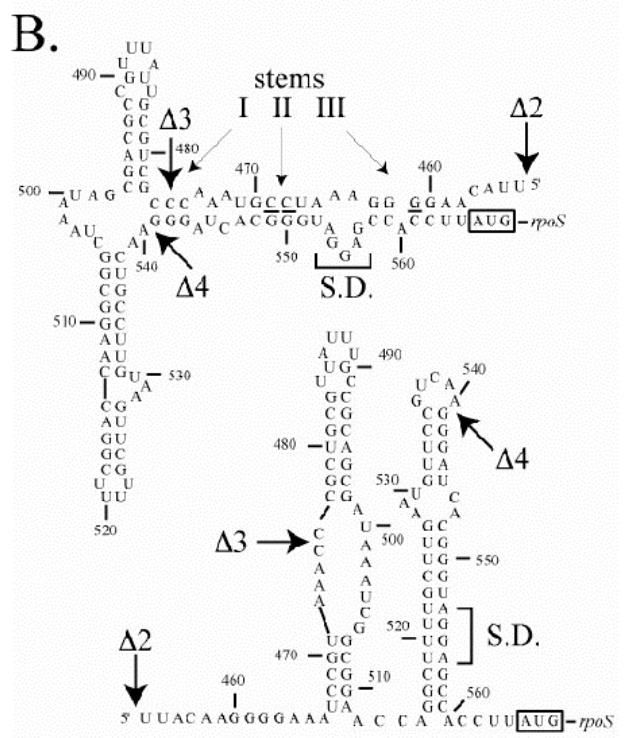

Alternative secondary structures in the rpoS RBS region. promoters. The upstream promoter cluster $\left(\mathrm{P}_{n l p D}\right)$, which is not thought to be regulated, serves both $n l p D$ and $r p o S$. The downstream promoter $\left(\mathrm{P}_{\text {rpos }}\right)$, is regulated and serves only rpos; its transcript includes an untranslated leader of $564 \mathrm{nt}$. The first nucleotide of the transcript from $\mathrm{P}_{r p o S}$, (as specified in the text) is taken as the basis for numbering used here (nt 1). The numbers used previously by us $(7,10)$ can be converted to this system by adding an offset of $341 \mathrm{nt}$ Most lac fusions used in this study have lac $Z$ placed to form either an operon or protein fusion at the EagI site within rpoS (codon 73). Other fusions, to codon 8 of rpoS, are so indicated in the text. Panel B shows two possible secondary structures for RNA including the end of the $n l p D$ coding sequence and the short intergenic region up to the AUG start codon of rpoS. The UAA stop codon terminating $n l p D$ lies at nt 500-502. The top structure is that proposed previously by us (7), and includes an upstream antisense element with three stems that can pair with a complementary sequence within the RBS, directly upstream of the rpoS AUG start codon. The Shine-Dalgarno (S.D.) sequence complementary to $16 \mathrm{~S}$ rRNA is also indicated. The lac UV5 promoter was used to drive expression of various constructs of two general types, as detailed in the text. The end-points of sequence derived from nlpD-rpoS included in these fusions can be described with reference to this figure as follows. Fusions for which the promoter is shown as "rpoS" include DNA starting from the ClaI site (panel A). Other fusions contain substitutions of the lacUV5 promoter followed by DNA starting from: nt 1 of the $\mathrm{P}_{\text {rpos }}$ transcript, the KpnI site, $\Delta 1$ (nt 344), $\Delta 2$ (nt 454), $\Delta 3$ (nt 477), or $\Delta 4$ (nt 541). For $\Delta 2$ through $\Delta 4$ included leader DNA sequences are shown in panel B. 


\section{Results}

Expression of rpoS-lac along the growth curve. Growth of cells for $\beta$-galactosidase assay was at $37^{\circ} \mathrm{C}$ (with one exception noted below) in LB medium. Cultures were started by a 1:500 dilution starting from overnight cultures grown under the same conditions. In $\mathrm{LB}$ medium at $37^{\circ} \mathrm{C}$ the generation time of the wild type $\left(\mathrm{relA}^{+} \mathrm{spoT}^{+}\right)$strain was 24 minutes, while the generation time of the otherwise isogenic ppGpp ${ }^{\mathrm{m}}$ mutant ( $\Delta$ relA $\Delta$ spoT) was 33 minutes (Fig. 4A). For each culture, the time at which stationary phase begins (designated $\mathrm{S}$ below and in data tables) was arbitrarily defined as 1 hour after the time at which the $\mathrm{OD}_{600}$ reached 0.5 . This definition compensates for the slower growth rate of the ppGp ${ }^{\mathrm{o}}$ mutant; it also allows the times for stationary phase sampling to be fixed while the culture is still in exponential phase. The point on the growth curve at $\mathrm{S}$ is very close to the inflection point between lines for exponential and stationary phases.

In a preliminary experiment we found that wild type cells carrying the rpoS-lac [pr] fusion, taken at densities between $\mathrm{OD}_{600}=0.01$ and $\mathrm{OD}_{600}=0.25$, showed nearly the same activity for $\beta$-galactosidase (data not shown). So as to maximize recovery, $\mathrm{OD}_{600}=0.25$ was chosen as the reference density for exponential phase, before the increase characterizing the transition into stationary phase. Plots of $\beta$ galactosidase activity determined as cells achieved stationary phase are shown in Fig $4 \mathrm{~B}$. The times

shown in this panel are slightly different than shown in panel A: the x-axis of the plot of the ppGpp mutant has been shifted to align it with wild type at $\mathrm{OD}_{600}=0.5$ (as well as $\mathrm{S}$ and subsequent points). 

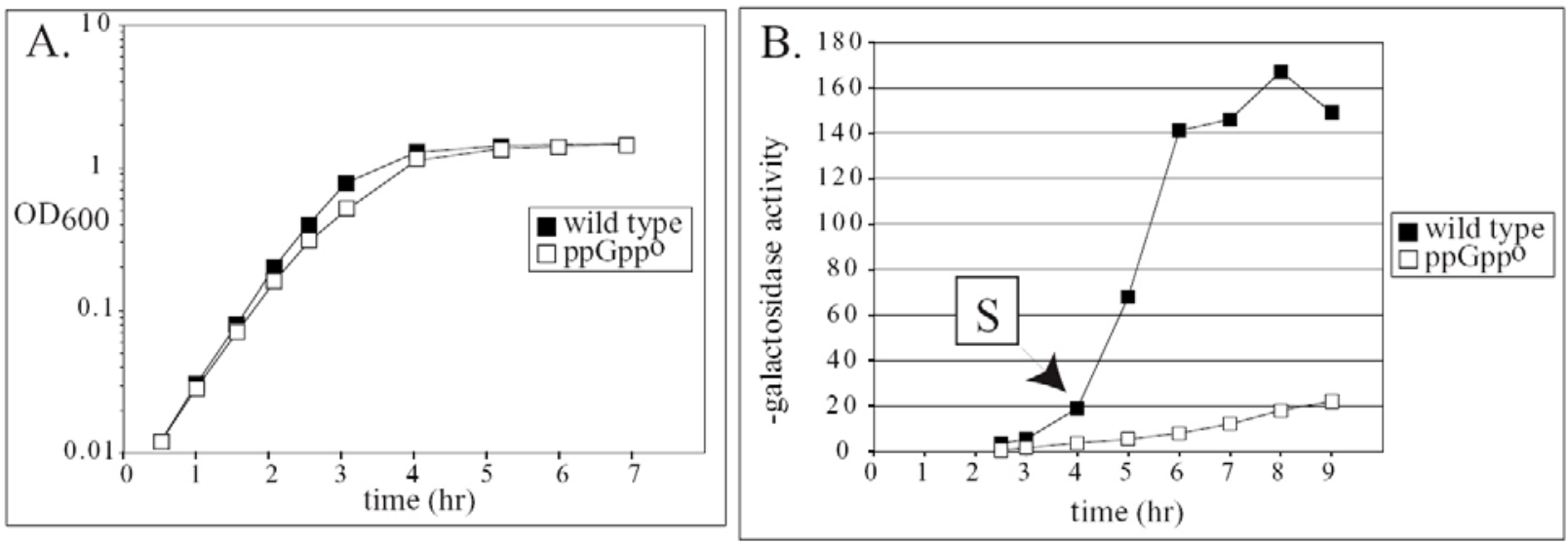

Figure 4. Expression of rpoS-lac [pr] as a function of growth phase.

A. Growth curve of the wild type strain carrying rpoS-lac [pr] (TE8197, filled squares) and its ppGpp ${ }^{0}$ mutant derivative (TE8199, open squares) in LB medium at $37^{\circ} \mathrm{C}$. As described in the text, stationary phase (S) was defined as one hour past the time at which the $\mathrm{OD}_{600}$ reached 0.5. In this experiment, for the wild type strain $\mathrm{S}=3 \mathrm{hr} 42 \mathrm{~min}$, and for the $\mathrm{ppGpp}^{\mathrm{o}}$ strain $\mathrm{S}=3 \mathrm{hr} 54 \mathrm{~min}$.

B. Cultures were grown as in panel A, and sampled at various times for assay of $\beta$-galactosidase. The first point for each curve corresponds to the time at which $\mathrm{OD}_{600}=0.25$. The $\mathrm{x}$-axes of the plots have been shifted slightly to align the points corresponding to $\mathrm{OD}_{600}=0.5$ at the $4 \mathrm{hr}$ mark. Each subsequent sample was taken at one hour intervals.

Our data are consistent with results reported in previous studies $(14,17)$. The wild type strain with rpoS-lac [pr] shows low but significant activity during exponential phase (Table 5), which then rapidly increases during the approach to and in early stationary phase. Maximum activity is achieved by $\mathrm{S}+2 \mathrm{hr}$ and is not increased by overnight growth (data not shown). The overall induction ratio is 35 - to 40-fold. In contrast to wild type, the ppGpp ${ }^{\circ}$ mutant has much lower expression of rpoS-lac at all times. The mutant shows about a 6-fold decrease compared to wild type even during exponential phase (Table 5), and the ratio of activity in the mutant compared to that in wild type is approximately the same during exponential phase and late stationary phase. It is only during early stationary phase that the ppGpp ${ }^{\mathrm{o}}$ mutant seems to be delayed in comparison to the increase seen in rpoS-lac expression for wild type. For 
the mutant, there is a gradual three-fold increase in $\beta$-galactosidase activity seen at late times, between $\mathrm{S}+2 \mathrm{hr}$ and $\mathrm{S}+5 \mathrm{hr}$ (Fig. 4B). 
Table 5. Effect of ppGpp on rpoS-lac and lac expression in vivo ${ }^{a}$.

\begin{tabular}{|c|c|c|c|c|c|c|c|c|c|c|c|}
\hline \multirow{3}{*}{$\begin{array}{l}\text { lac fusion } \\
\text { Promoter }\end{array}$} & \multirow{3}{*}{ reporter } & \multirow[b]{3}{*}{ type } & \multicolumn{6}{|c|}{$\beta$-galactosidase activity (U) } & \multirow{2}{*}{\multicolumn{3}{|c|}{$\begin{array}{l}\text { Activity ratio, } \\
\text { mutant/wild type }\end{array}$}} \\
\hline & & & \multicolumn{3}{|c|}{ Wild type } & \multicolumn{3}{|c|}{ Mutant (ppGpp ${ }^{\circ}$ ) } & & & \\
\hline & & & $\mathrm{E}$ & $\mathrm{S}+3$ & $\mathrm{~S}+3 / \mathrm{E}^{\mathrm{b}}$ & $\mathrm{E}$ & $\mathrm{S}+3$ & $\mathrm{~S}+3 / \mathrm{E}$ & $\mathrm{E}$ & $\mathrm{S}+3$ & $\mathrm{~S}+3 / \mathrm{E}$ \\
\hline rpos & rpos-lac & Protein & 3.4 & 120 & 35 & 0.54 & 5.1 & 9.4 & 0.16 & 0.04 & 0.27 \\
\hline rpos & rpos-lac & Operon & 35 & 510 & 15 & 11 & 50 & 4.5 & 0.31 & 0.10 & 0.31 \\
\hline lacUV5 & rpos-lac & Protein & 5.3 & 71 & 13 & 2.1 & 7.6 & 3.6 & 0.40 & 0.11 & 0.27 \\
\hline lacUV5 & rpos-lac & Operon & 100 & 330 & 3.3 & 64 & 130 & 2.0 & 0.64 & 0.39 & 0.61 \\
\hline lacUV5 & rpoS-lac $(+1)$ & Protein & 10 & 130 & 13 & 3.2 & 25 & 7.8 & 0.32 & 0.19 & 0.60 \\
\hline rpos & rpoS-lac $(\operatorname{codon} 8)^{b}$ & Protein & 4 & 130 & 32 & 0.62 & 13 & 21 & 0.15 & 0.10 & 0.64 \\
\hline lacUV5 & lac & Operon & 120 & 160 & 1.3 & 81 & 80 & 0.99 & 0.67 & 0.50 & 0.74 \\
\hline
\end{tabular}

${ }^{a}$ Exponential-phase (E) and stationary-phase ( $\mathrm{S}+3$ ) samples are defined in text. Values are averages with a variation of $<20 \%$, with one exception noted in the text. The $\mathrm{S}+3 / \mathrm{E}$ induction ratio was calculated as the activity of the $\mathrm{S}+3$ sample divided by that of the $\mathrm{E}$ sample.

${ }^{b}$ all other fusions were to codon 73 of rpoS. 
Table 6. Role of the antisense element in stationary phase induction and ppGpp effects on rpoS-lac ${ }^{a}$.

\begin{tabular}{|c|c|c|c|c|c|c|c|c|c|c|}
\hline \multirow{2}{*}{\multicolumn{2}{|c|}{ lac fusion }} & \multicolumn{6}{|c|}{$\beta$-galactosidase activity (U) } & \multirow{2}{*}{\multicolumn{3}{|c|}{$\begin{array}{c}\text { Activity ratio, } \\
\text { mutant / wild type }\end{array}$}} \\
\hline & & \multicolumn{3}{|c|}{ wild type } & \multicolumn{3}{|c|}{ mutant (ppGpp ${ }^{\mathrm{O}}$ ) } & & & \\
\hline \multicolumn{2}{|c|}{ Promoter Reporter } & $\mathrm{E}$ & $\mathrm{S}+3$ & $\mathrm{~S}+3 / \mathrm{E}$ & $\mathrm{E}$ & $\mathrm{S}+3$ & $\mathrm{~S}+3 / \mathrm{E}$ & $\mathrm{E}$ & $\mathrm{S}+3$ & $\mathrm{~S}+3 / \mathrm{E}$ \\
\hline \multirow[t]{5}{*}{ lacUV5 } & $r p o S-l a c^{\mathrm{a}}$ & 5.7 & 82 & 14 & 2.1 & 6.7 & 3.2 & 0.37 & 0.08 & 0.22 \\
\hline & rpoS-lac, $\Delta 1$ & 4.7 & 65 & 14 & 1.8 & 7.5 & 4.2 & 0.38 & 0.12 & 0.30 \\
\hline & rpoS-lac, $\Delta 2$ & 3.1 & 34 & 11 & 1.6 & 5.7 & 3.6 & 0.52 & 0.17 & 0.32 \\
\hline & rpoS-lac, $\Delta 3$ & 13 & 73 & 5.6 & 8.4 & 62 & 7.4 & 0.65 & 0.85 & 1.3 \\
\hline & rpoS-lac, $\Delta 4$ & 8.3 & 40 & 4.8 & 5.2 & 30 & 5.8 & 0.63 & 0.75 & 1.2 \\
\hline rpos & rpoS-lac & 3.1 & 120 & 39 & 0.66 & 8.8 & 13 & 0.21 & 0.07 & 0.34 \\
\hline rpos & rpoS-lac C469G & 7.7 & 210 & 27 & 1.4 & 47 & 34 & 0.18 & 0.22 & 1.23 \\
\hline rpos & rpoS-lac G549C & 14 & 360 & 26 & 3.3 & 29 & 8.8 & 0.24 & 0.08 & 0.34 \\
\hline rpos & rpoS-lac C469G G549C & 6.5 & 170 & 26 & 1.4 & 7.9 & 5.6 & 0.22 & 0.05 & 0.22 \\
\hline
\end{tabular}

${ }^{a}$ All fusions listed in this table are protein fusions. See Table 5 , footnote ${ }^{a}$, for definitions. 
The defect in stationary phase rpoS-lac expression seen for the ppGpp ${ }^{\mathrm{o}}$ mutant was previously reported by others (17). However, we also find a quantitatively similar exponential phase defect of rpoS-lac expression in the same mutant. We would distinguish between the defect in basal (or constitutive, non-regulated) expression and a true regulatory defect. The basal defect of the ppGpp ${ }^{\mathrm{O}}$ mutant is about six-fold; while the regulatory defect for stationary phase induction seen at $\mathrm{S}+3 \mathrm{hr}$ is approximately three-fold. These results indicate that stationary phase induction of $r p o S$ is not simply due to increased ppGpp during stationary phase because it still occurs normally even in the complete absence of ppGpp. Accumulation of RpoS in the ppGpp ${ }^{\mathrm{o}}$ mutant after overnight incubation in stationary phase is consistent with previous studies $(14,17)$. Of course, these experiments do not exclude the possibility that ppGpp might play a quantitatively larger regulatory role under other conditions.

Promoter substitution. Expression of rpoS-lac was measured from constructs in which the lacUV5 promoter was substituted for the native rpoS promoters (10). The lacUV5 promoter was chosen because its activity was previously reported to be completely independent of ppGpp $(1,2)$; also, its activity is only slightly increased during stationary phase (see the last entry of Table 5). When expression of rpoS-lac [pr] was driven by lacUV5p, a strong stationary phase induction still occurred (induction ratio of 13-fold; Table 5). The stationary phase induction was smaller by a factor of 3 than that seen with the native promoters, suggesting that the native promoters contribute to stationary phase induction to this extent. We also compared the effect of stationary phase on expression of rpoS-lac protein and operon fusions driven by either native or lacUV5 promoters. Combined, the results support the idea that stationary phase controls multiple stages in rpoS expression. (i) a role for translational 
regulation is suggested by the larger induction ratio when [pr] fusions are compared with [op] fusions, whether driven by the native promoters or by lacUV5p; (ii) a role for the native rpoS promoters is suggested by the smaller induction ratio when lacUV5p is substituted in either [op] or [pr] fusion contexts; and (iii) a role for post-initiation transcriptional control is suggested by the residual stationary phase induction seen in the lacUV5p-rpoS-lac [op] construct.

Expression of rpoS-lac [pr] from the lacUV5 promoter was decreased significantly in the ppGpp ${ }^{\mathrm{o}}$ mutant background (Table 5). The time course for both mutant and wild type (data not shown) was similar to that seen when the construct was driven by the native promoters (Fig 4B). Expression was lower for the mutant at all times, but with a gradual increase in expression in the mutant until $\mathrm{S}+5$ hr. To detect the regulatory defect of the mutant, we chose $\mathrm{S}+3 \mathrm{hr}$ as the time of sampling for stationary phase (Table 5). Selected stationary phase induction ratios calculated in Table 5 are plotted in Fig 5 . It can be seen that the regulatory defect of the $\mathrm{ppGpp}^{\mathrm{o}}$ mutant is similar for rpoS-lac protein and operon fusions, as well as for protein fusions whether driven by the native promoters or lacUV5p. This suggests that neither the promoter nor translation-level control are significant targets for the regulatory effect of ppGpp.

In contrast to its regulatory effects, the defect in basal expression of the ppGpp ${ }^{\mathrm{o}}$ mutant seems to be distributed over several elements. For example, whether comparing exponential or stationary phase values, the biggest defect is shown by rpoS-lac [pr] driven by the native promoters. The relative defect is less for either a lacUV5 promoter substitution or an operon fusion. The former result is consistent with direct measurement of rpoS mRNA 5' ends (17). We also found that lacUV5 promoter activity 
was somewhat lower in a ppGpp ${ }^{\mathrm{o}}$ strain---as much as 2-fold lower in stationary phase (Table 5). This effect of ppGpp in the control, while small, makes the significance of other small effects uncertain (in particular, the effect of ppGpp in the lacUV5p-rpoS-lac [op] construct).

\section{Figure 5. Nature of the $\mathrm{ppGpp}{ }^{0}$ regulatory defect.}

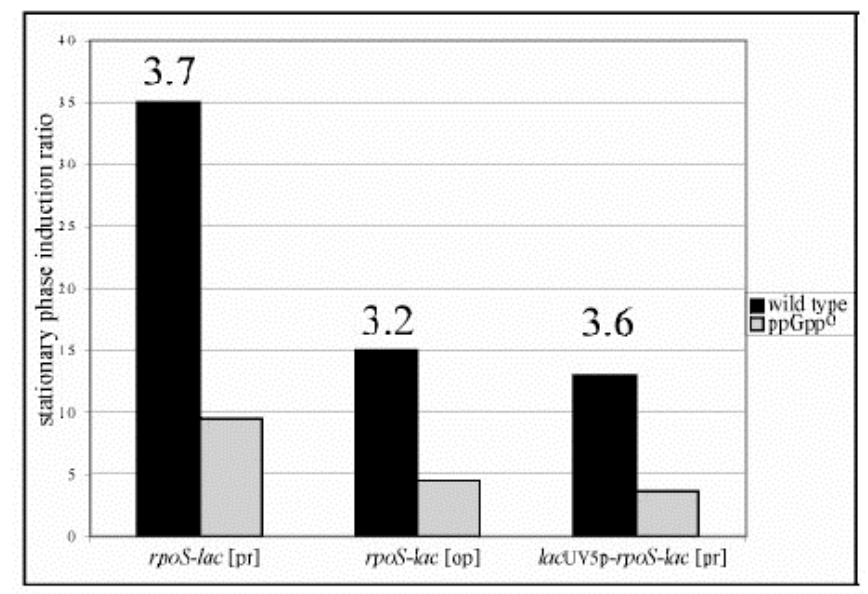

Various rpoS-lac fusion strains were grown and assayed for $\beta$-galactosidase as reported in Table 5. The stationary phase induction ratio (expression at $\mathrm{S}+3$ divided by that at $\mathrm{E} 1$ ) is plotted in bar format for both mutant and ppGpp ${ }^{\mathrm{o}}$ versions of each fusion. The ratio (value in wild type divided by that in $\mathrm{ppGpp}^{\mathrm{o}}$ mutant) of the stationary phase induction ratios is shown above the bar for each wild type strain. The strains used are listed in Table 8.

The reference lacUV5p constructs were made using a convenient KpnI site (10); this strategy removed $72 \mathrm{nt}$ of $r p o S$ leader sequence. Therefore an additional lacUV5p construct was made which includes all nucleotides of the native transcript $($ denoted +1$)$; it is regulated identically to the reference construct in stationary phase, although it seems to be somewhat less sensitive to ppGpp during stationary phase. The significance of this difference is not understood at present. Finally, one fusion was tested in which lac is joined to rpoS at codon 8 (rather than to codon 73 as in all other rpoS fusions used to this 
point). The codon 8 fusion (driven by the native rpoS promoters) was regulated normally by stationary phase and was highly responsive to ppGpp.

Deletion mutants. Deletion analysis was used to define the region(s) of the rpoS leader which are required for stationary phase induction and the response to wild type ppGpp levels (Table 6). Cell samples from wild type and $\mathrm{ppGpp}^{\mathrm{o}}$ backgrounds were collected in both exponential and stationary phase, and stationary phase induction ratios calculated. For each construct, the promoter was lacUV5p. All constructs share a common transcribed leader of $60 \mathrm{nt}$ (partially derived from lac); the only difference between constructs is the amount of rpoS sequence that is retained. The first entry in Table 6 shows the reference construct (substitution at the KpnI site, construct $\mathrm{K}$ from reference 10). The deletions ( $\Delta 1$ through $\Delta 4$ ) extend progressively closer to the rpoS ATG initiation codon, as shown in Fig 3. The $\Delta 4$ construct retains only $24 \mathrm{nt}$ of $r p o S$ sequence upstream of the rpoS ATG initiation codon.

There is no significant difference in either induction ratio or ppGpp response between the $\mathrm{K}$ construct and $\Delta 1$, and there is only a slight decrease in induction ratio as the deletion is extended in $\Delta 2$. However, a clear breakpoint can be seen between $\Delta 2$ and $\Delta 3$. It is striking that the two deletions that extend farther downstream ( $\Delta 3$ and $\Delta 4$ ) lack the ppGpp-dependence shown by the reference construct and upstream deletions. Selected data from Table 6 highlighting the effect of ppGpp on induction ratio are shown in a bar plot in Fig. 6, panel A. The plot clearly shows that the $\Delta 3$ and $\Delta 4$ deletions retain substantial stationary phase induction but have lost the ppGpp response. The region between the $\Delta 2$ and $\Delta 3$ deletion endpoints required for this facet of the ppGpp response includes the upstream antisense element. There is also approximately a two-fold decrease in the stationary phase induction ratio 
between $\Delta 2$ and $\Delta 3$. However, a substantial (five-fold) stationary phase induction is retained in both the $\Delta 3$ and $\Delta 4$ deletions, despite the fact that they do not respond to ppGpp.
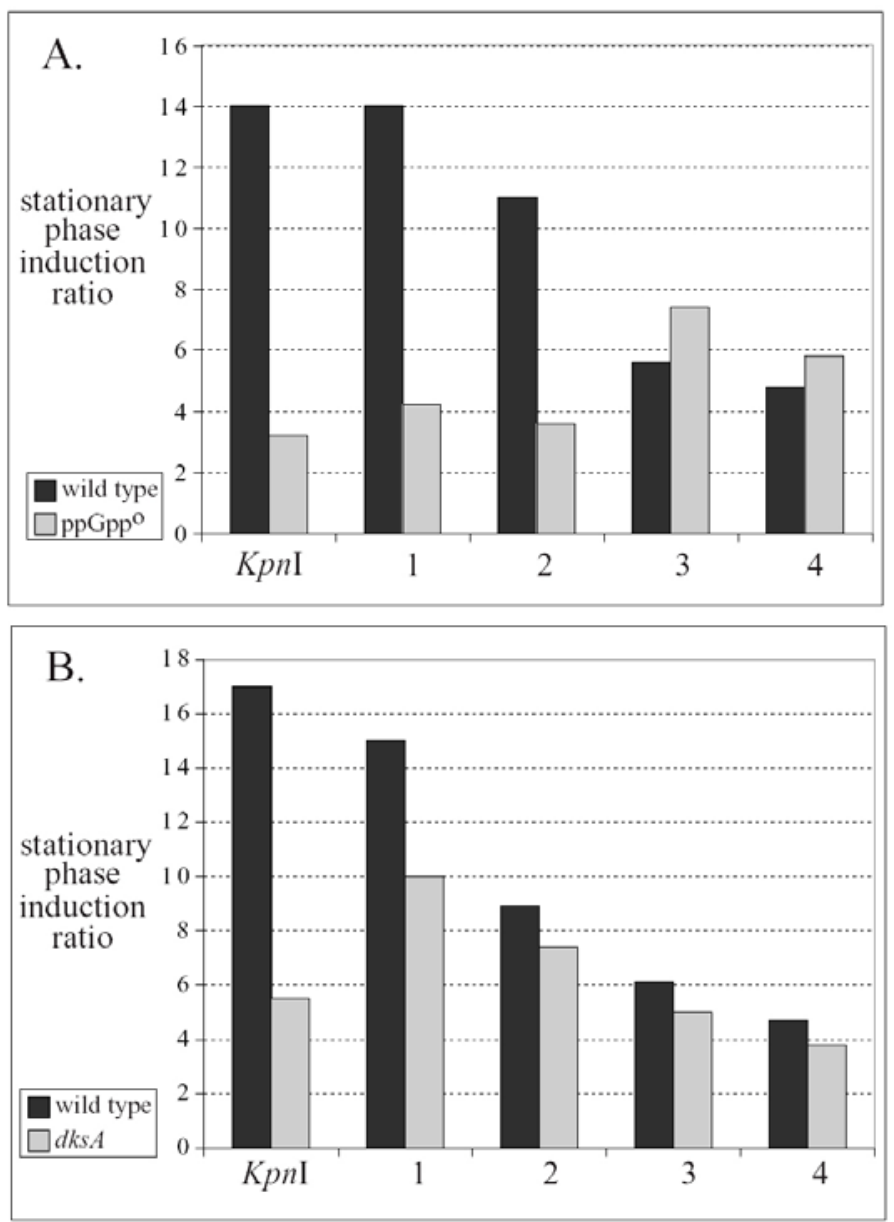

Figure 6. Deletion mapping of sequences required for $\operatorname{ppGpp}^{0}$ (A) and dksA (B) mutation effects on rpoS expression.

Various rpoS-lac fusion strains, all driven by the lacUV5 promoter but with varying amounts of the upstream rpoS leader, were assayed for $\beta$-galactosidase during exponential phase and stationary phase. Stationary phase induction ratios were calculated and plotted here. The underlying data for the ppGpp ${ }^{0}$ strain are from Table 6. The strains used are listed in Table 8.

In a previous study we described a number of mutations which affect the upstream antisense element or its complementary sequence within the RBS of rpoS mRNA, immediately upstream of the 
rpoS ATG initiation codon (7). Two of these mutations were examined here: C469G and G549C, which alter the antisense (top) and sense (bottom) components of stem II respectively (see the legend to Fig. 3 for details). We chose these two mutations because, as tested in S. enterica, in the double mutant the resulting rpoS-lac expression phenotype is indistinguishable from wild type and very different from each single mutant, providing strong genetic support for the proposed inhibitory mRNA secondary structure. Furthermore, mutations at adjacent positions share these properties (unpublished data).

Here, it is striking that the $\mathrm{C} 469 \mathrm{G}$ mutation allows high stationary phase expression and restores a normal induction ratio of $r p o S$ in the $\operatorname{ppGpp}^{\mathrm{o}}$ background (Table 6). This lesion clearly relieves most of the ppGpp requirement of rpoS. Furthermore, the C469G/G549C double mutant restores ppGpp dependence, which is consistent with the model that ppGpp-dependence can be counteracted by blocking interaction of the antisense element with the RBS. However, since the G549C single mutant should loosen the secondary structure as effectively as C469G, the model predicts that G549C should relieve the ppGpp requirement of $r p o S$, yet this prediction is not fulfilled.

Additionally, when comparing E. coli with S. enterica, the phenotype of the compensatory double mutant is not always as clear-cut in E. coli. Particularly in a wild type $\left(\mathrm{relA}^{+} \mathrm{spoT}^{+}\right)$strain background, the C469G/G549C double mutant does not exhibit a fully wild type phenotype but rather is intermediate between C469G and wild type (Table 6): compare 120 units (wild type), 170 units (double

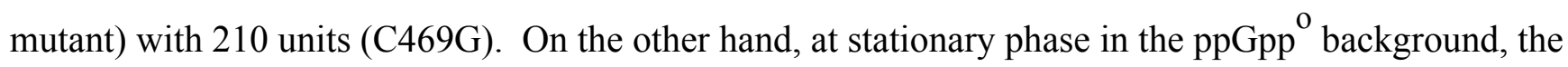
phenotype of the double mutant is more convincingly like that of wild type: compare 8.8 units (wild type), 7.9 units (double mutant) with 47 units (C469G) and 29 units (G549C). The partial suppression 
observed in the double mutant in a wild type background leaves some question whether the observed phenotypes result simply from a failure of antisense element interaction with the RBS. They might reflect an additional sequence-specific interaction. Nevertheless it is clear that $\mathrm{C} 469 \mathrm{G}$ eliminates a requirement for ppGpp to observe normal stationary phase induction and the requirement for ppGpp is restored in the $\mathrm{C} 469 \mathrm{G} / \mathrm{G} 549 \mathrm{C}$ double mutant.

Effect of dksA. A dksA insertion in S. enterica was reported to decrease stationary phase induction of an rpoS-lac protein fusion in supplemented minimal medium (41). In other work, we have found that a dksA deletion in E. coli almost completely blocks induction of rpoS by elevated ppGpp during exponential growth in LB medium (8). Therefore, we tested whether the effect of $d k s A$ on rpoSlac $[\mathrm{pr}]$ expression exhibits the same sequence requirements as observed for the regulatory defect of $\operatorname{ppGpp}^{\mathrm{O}}$.

Expression of $r p o S-l a c[p r]$ in the $\Delta d k s A$ ::tet mutant was not detectably different from that in the wild type during exponential phase but was one-third to two-thirds of the wild type level in stationary phase,, measured either with the wild type fusion or with lacUV5p constructs (data not shown). The regulatory defect of $d k s A$, as reflected in the stationary phase induction ratio, was larger for fusions carrying more upstream sequence (the KpnI fusion in Fig. 6B). Only a small regulatory effect of $d k s A$ was seen for $\Delta 1$, and this was nearly completely lost for $\Delta 2$ and with reporters deleted for the antisense element ( $\Delta 3$ and $\Delta 4)$. This pattern is similar to that for $h f q$, where its effect on $r p o S$ expression requires upstream sequences (10; see below), and differs from the requirements for ppGpp effects as described above. 
We have found similar results for $d k s A$ in S. enterica in LB medium (unpublished data). A more substantial effect for $d k s A$ can be observed in S. enterica in minimal medium supplemented with amino acids (41, unpublished data); this difference may be related to the multiple auxotrophy of $d k s A$ mutants in both E. coli and S. enterica.

Other trans-acting factors. In addition to the above studies utilizing the $\mathrm{ppGp}^{\mathrm{o}}$ background $(\Delta r e l A \Delta s p o T)$ and a $d k s A$ mutation, we also examined the effects on stationary phase induction of rpoSlac for mutations in four other genes. The mutations tested were newly constructed insertion/deletions of barA (29), or ppkx (36), and existing mutations affecting $d s r A$ (deletion; 39), and $h f q(\Omega-K m$ insertion; $6,28,40)$.

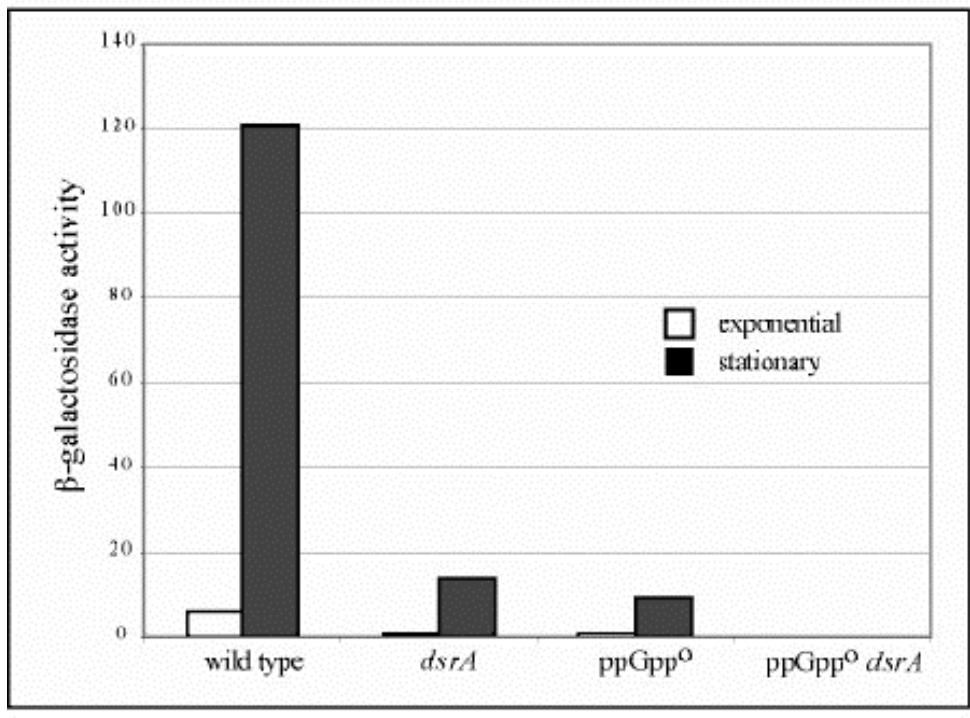

Figure 7. Additive effect of $\mathrm{ppGpp}^{\circ}$ and a dsrA mutation.

Activity of $\beta$-galactosidase from exponential $\left(\mathrm{OD}_{600}=\right.$ $0.25)$ and stationary phase $(\mathrm{S}+3 \mathrm{hr})$ cultures are given. The strains used are listed in Table 8. 
Previously, a barA insertion (of $\lambda$ placMu53) in MC4100 was shown to reduce RpoS by Western blot and rpoS RNA by Northern blot (29). A ppk ppx mutant in the JM101 background was reported to have decreased HPII catalase (under RpoS control), and plasmid-encoded yeast PPX1 polyphosphatase interfered with katE and rpoS induction as measured by Western blot and lac operon fusion, consistent with positive regulation of rpoS by polyphosphate (36).

For the first two mutations (barA and ppkx), we found that the effects on rpoS-lac expression during growth in LB medium were small (data not shown). No significant effect of $\triangle b a r A$ ::tet was observed for katE-lac [op] expression in either the MC4100 or MG1655 backgrounds; rpoS-lac [pr] expression was either unchanged (MC4100) or elevated (MG1655). The $\Delta p p k x:$ :tet strain expressed rpoS-lac [pr] at about $75 \%$ of the wild type level in stationary phase, whereas the ppGpp $^{\mathrm{o}}$ derivative was about $10 \%$ of wild type in the same experiment. The $\Delta p p k x:$ :tet mutant showed an rpoS-lac [pr] induction ratio of 26 -fold, versus 34 -fold for wild type. These small effects are difficult to interpret.

The dsrA gene encodes a small, untranslated RNA which is proposed to base pair with the upstream antisense element of rpoS, thereby freeing the RBS to be engaged by ribosomes for translation. Strong genetic evidence supports this model including the behavior of several sets of compensatory mutations (23). Sledjeski et al showed that in wild type cells RpoS abundance and rpoS-lac expression increase dramatically at lower growth temperatures. One major effect of the dsrA mutation is that this low temperature response is lost (39). At $37^{\circ} \mathrm{C}$, there is no effect of a dsrA mutation on rpoS-lac 
expression in either exponential or stationary phase, whether in the MC4100 background (33) or in MG1655 (our unpublished results). DsrA RNA is not required for stationary phase induction under these conditions.

In order to see the effect of loss of $d s r A$, cells were grown at $30^{\circ} \mathrm{C}$. At this temperature, wild type cells with the standard fusion (construct A) showed a normal stationary phase induction (Fig. 7).

Both the dsrA mutant and the $\operatorname{ppGpp}^{\circ}$ mutant showed a substantial reduction in rpoS-lac expression, but only modest effects were observed on the stationary phase induction ratio. A mutant lacking both dsrA and ppGpp showed a more severe phenotype in which rpoS-lac expression was almost completely absent. Because both the ppGpp ${ }^{\mathrm{o}}$ and dsrA backgrounds show severe (10-fold) effects on rpoS-lac, we suggest that during growth at $30^{\circ} \mathrm{C}$ most $r p o S$ expression requires both ppGpp and DsrA RNA (whether directly or indirectly). We interpret the additive effect of the combined mutations by suggesting that the two effectors act independently at least in part---ppGpp does not simply act by changing DsrA level or activity. However, we emphasize the finding that during growth at $37^{\circ} \mathrm{C}, d s r A$ function does not affect rpoS expression, and even at $30^{\circ} \mathrm{C}$, dsrA mutants show a stationary phase induction ratio of about 23 fold.

We also compared the effect of $\Delta d s r A$ in $\Delta 1$ through $\Delta 4$ (data not shown). The model of DsrA acting as an anti-antisense RNA predicts that deletions of the antisense element should not respond to lack of DsrA. As predicted, the $\Delta d s r A$ deletion does not affect $\Delta 3$ and $\Delta 4$. 
The last trans-acting regulator that we've investigated is $h f q$. We found that, as in S. enterica (10), the $\Delta 2$ construct does not respond to loss of $h f q$ and the $\Delta 1$ construct shows only a modest effect (data not shown). Furthermore, the stationary phase induction ratio of an $h f q$ mutant is very similar to the $h \mathrm{fq}^{+}$control, whether the fusion is rpoS-lac [pr] driven by the native promoters, or the $\Delta 1$ or $\Delta 2$ constructs, driven by lacUV5p (data not shown). Therefore, Hfq cannot be a mediator of stationary phase induction. Finally, many mutations identifying "regulators" of rpoS expression have been reported to have no effect in an $h f q$ mutant background. This set includes: hns (28); stpA; galU, pgi, and pgm (5); and oxyS (44). Similarly, a leuO mutation makes no difference in the absence of $d s r A$ (16a). Since stationary phase induction is normal in an $h f q$ mutant, and these mutations presumably

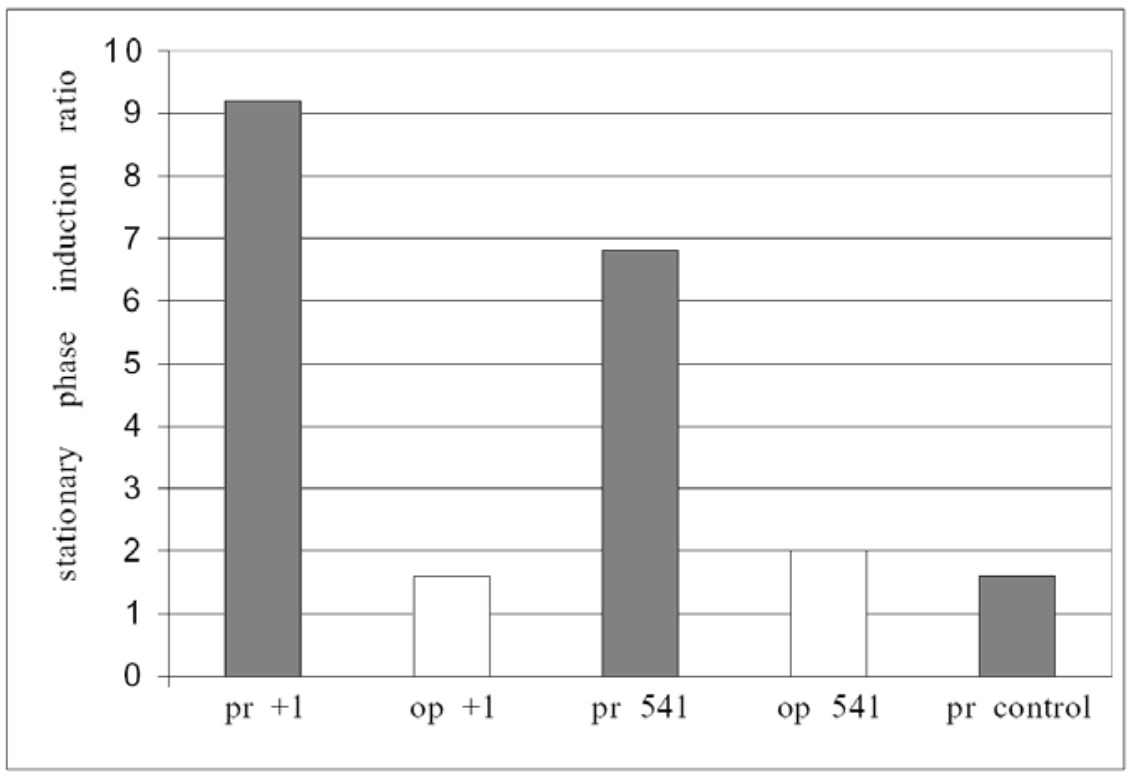

pr 541 transcript AAUUGGGAUCACGGGUAGGAGCCACCUU AUG-rpoS-lac pr control transcript AAUUUCACACAGGAAACAGCU AUG-rpoS-lac

affect RpoS secondarily through their effects on $h f q$, these factors cannot regulate stationary phase induction.

Figure 8. Stationary phase regulation requires the wild type rpoS RBS. Various rpoS-lac fusion strains driven by the lacUV5 promoter were grown and assayed for $\beta$-galactosidase. The stationary phase induction ratio (expression at $\mathrm{S}+3$ divided by that at E1) is plotted in bar format for protein (pr) or operon (op) versions of each fusion. Fusions marked +1 contain the entire $564 \mathrm{nt}$ rpoS leader; those marked 541 contain only $24 \mathrm{nt}$ upstream of the AUG start codon. The pr control transcript sequence is compared to the pr 541 transcript below the bar graph. In each fusion, codon 8 of the rpoS sequence is joined to lac. The strains used are listed in Table 8. 
Stationary phase regulation localized to the rpoS RBS region. As shown in Table 6 and Fig. 6 , a substantial stationary phase induction can be observed even with the $\Delta 4$ construct, driven by lacUV5 promoter, which contains only $24 \mathrm{nt}$ upstream of the rpoS ATG initiation codon. All lac fusion constructs used so far have contained rpoS coding sequences extending to codon 73 . We wondered whether this stationary phase response would be retained with lac fusions made to upstream sites, and whether operon fusions would show a difference from protein fusions. To make the new constructs, we used the $\lambda$ red recombination method (43) to position the lacUV5 promoter precisely at various sites. We chose to retain the first $4 \mathrm{nt}$ of the lac transcript (AAUU) in these fusions. Otherwise identical operon and protein fusion constructs were made by simply transforming the fusion fragment into a different host strain (see Materials and Methods for details). The results (Fig. 8) show that this aspect of the stationary phase response is seen with protein but not with operon fusions (indicating a translationlevel defect) and does not require rpoS sequences downstream of codon 8 . The fusion labeled pr control in Fig. 8, substitutes the lac RBS (not including operator sequences), for the rpoS RBS.

\section{Discussion.}

Ever since the discovery by Gentry et al that mutants lacking ppGpp are deficient in RpoS after growth to stationary phase in rich medium (14), a role for ppGpp in RpoS regulation has been an attractive unifying hypothesis. Thus, ppGpp control of RpoS might explain not only induction of RpoS during growth into stationary phase, but also growth rate regulation, induction by limitation for single nutrients and by other stresses such as challenge with high salt, and even induction during growth within 
eukaryotic host cells. Here, the sensitivity obtained by using lac as a reporter for $r p o S$ expression allows a clear demonstration that $\mathrm{ppGp}^{\mathrm{o}}$ mutants are just as defective during exponential as in late stationary phase and therefore, we suggest that ppGpp should be considered mainly as a basal and not a regulatory factor.

This conclusion should be qualified. First, $\mathrm{ppGpp}^{\mathrm{o}}$ mutants do show a delay in the rate of increase in RpoS during stationary phase, so that relatively early (at the time we define as $\mathrm{S}+3 \mathrm{hr}$ ), there is a modest regulatory defect which is made up by a slow, late accumulation. Second, it is conceivable that ppGpp is regulatory in stationary phase in wild type cells, but a redundant mechanism operates in the ppGpp ${ }^{0}$ mutant. Also, the delay of the mutant in achieving stationary phase, and halting protein synthesis, could allow more time for RpoS to accumulate or might trigger some type of compensatory increase. Finally, a regulatory role for ppGpp under other conditions is not ruled out. Preliminary experiments indicate that osmotic shock can still induce rpoS normally even in a ppGpp ${ }^{0}$ host (unpublished data). But ppGpp control might explain the large difference between rpoS expression in rich and minimal medium. The complex nutritional defect of $\operatorname{ppGpp}^{\circ}$ mutants makes that idea difficult to test.

Analysis of rpoS-lac operon and protein fusions, as well as promoter substitutions and deletions, should allow us to suggest one or more mechanisms of ppGpp action in this system. The total effect is robust: expresssion of rpoS-lac [pr] is ca. 25 -fold higher in wild type than for the ppGpp ${ }^{0}$ mutant at $\mathrm{S}+3$ hr (Table 5). However, this large effect is distributed in small installments over several targets. The 
basal increase in expression involves: (i) an effect lost when transcription is from the lacUV5 promoter (consistent with primer extension results in reference17), (ii) an effect seen with a protein fusion but not an operon fusion, and (iii) a target, probably transcription elongation, which is distinct from the first two. In contrast, the regulatory effect of ppGpp apparently involves the antisense element (Table 5 and Fig. 6) yet paradoxically, is still visible with an operon fusion and thus presumably involves transcription elongation (Fig. 5). The distributed nature of ppGpp's targets complicates analysis by combining several small, presumably multiplicative effects.

Another area of uncertainty involves the question whether high levels of ppGpp, achieved by inducing a truncated ribosome-independent RelA protein during exponential growth, alter rpoS expression in the same way as the effects seen here by use of a ppGpp ${ }^{\circ}$ mutant (14). Recent work shows that overproduction of ppGpp dramatically increases RpoS protein synthesis with little change in the amount of rpoS RNA (8). Our results, with only a small role for translational control, seem inconsistent with this. One resolution is to suppose that ppGpp overproduction affects rpoS by a different mechanism. In support of this interpretation, $d k s A$ function is required for $p p G p p$ overproduction to induce rpoS (8), yet stationary phase regulation is normal in a $d k s A$ mutant. Furthermore, the sequences required for $d k s A$ to affect rpoS expression in our experiments are different than those required for ppGpp regulation (Fig. 6).

The most surprising conclusion from these experiments is that much of stationary phase regulation is retained in lacUV5p-driven fusions that retain only $24 \mathrm{nt}$ upstream of the rpoS AUG initiation codon and an additional 7 codons downstream (Fig. 8). This effect of sequences close to the 
ribosome binding site is independent of the antisense element, ppGpp, and all known trans-acting regulators of rpoS including $d s r A$ and $d k s A$.

\section{Acknowledgements.}

We are grateful to Mike Cashel for many helpful discussions, and to individuals cited in the text, for bacterial strains.

\section{Literature Cited}

1. Barker, M., T. Gaal, C. A. Josaitis, and R. L. Gourse. 2001. Mechanism of regulation of transcription initiation by ppGpp. I. Effects of ppGpp on transcription initiation in vivo and in vitro. J. Mol. Biol. 305:673-688.

2. Barker, M., T. Gaal, and R. L. Gourse. 2001. Mechanism of regulation of transcription initiation by ppGpp. I. Models for positive control based on properties of RNAP mutants and competition for RNAP. J. Mol. Biol. 305:689-702.

3. Benjamin, W. H., Jr., X. Wu, and W. E. Swords. 1996. The predicted amino acid sequence of the Salmonella typhimurium virulence gene $\mathrm{mviA}^{+}$strongly indicates that MviA is a regulator protein of a previously unknown S. typhimurium response regulator family. Infect. and Immun. 64:2365-2367.

4. Berkowitz, D., J. M. Hushon, H. J. Whitfield, J. Roth, and B. N. Ames. 1968. Procedure for identifying nonsense mutants. J Bacteriol. 96:215-220.

5. Bohringer, J., D. Fischer, G. Mosler, and R. Hengge-Aronis. 1995. UDP-glucose is a potential intracellular signal molecule in the control of expression of $\sigma^{\mathrm{S}}$ and $\sigma^{\mathrm{S}}$-dependent genes in Escherichia coli. J. Bacteriol. 177:413-422.

6. Brown, L., and T. Elliott. 1996. Efficient translation of the RpoS sigma factor in Salmonella typhimurium requires Host Factor I, an RNA-binding protein encoded by the $h f q$ gene. J. Bacteriol. 178:3763-3770. 
7. Brown, L., and T. Elliott. 1997. Mutations that increase expression of the rpoS gene and decrease its dependence on $h f q$ function in Salmonella typhimurium. J. Bacteriol. 179:656-662.

8. Brown, L., D. Gentry, T. Elliott and M. Cashel. 2002. DksA affects ppGpp induction of RpoS at a translational level. J. Bacteriol, in press.

9. Chen, C. Y., L. Eckmann, S. J. Libby, F. C. Fang, S. Okamoto, M. F. Kagnoff, J. Fierer, and D. G. Guiney. 1996. Expression of Salmonella typhimurium rpoS and rpoS-dependent genes in the intracellular environment of eukaryotic cells. Infect. Immun. 64:4739-4743.

10. Cunning, C., L. Brown, and T. Elliott. 1998. Promoter substitution and deletion analysis of upstream region required for rpoS translational regulation. J. Bacteriol. 180:4564-4570.

11. Cunning, C. and T. Elliott. 1999. RpoS synthesis is growth rate regulated in Salmonella typhimurium, but its turnover is not dependent on acetyl phosphate synthesis or PTS function. J. Bacteriol. 181:4853-4862.

12. Elliott, T. 1992. A method for constructing single-copy lac fusions in Salmonella typhimurium and its application to the hemA-prfA operon. J. Bacteriol. 174:245-253.

13. Fang, F. C., S. J. Libby, N. A. Buchmeier, P. C. Loewen, J. Switala, J. Harwood, and D. G. Guiney. 1992. The alternative $\sigma$ factor KatF (RpoS) regulates Salmonella virulence. Proc. Natl. Acad. Sci. USA 89:11978-11982.

14. Gentry, D. R., V. J. Hernandez, L. H. Nguyen, D. B. Jensen, and M. Cashel. 1993. Synthesis of the stationary-phase sigma factor $\sigma^{\mathrm{S}}$ is positively regulated by ppGpp. J. Bacteriol. 175:79827989.

15. Hengge-Aronis, R. 1996. Regulation of gene expression during entry into stationary phase, p.1497-1512. In F.C. Neidhardt, R. Curtiss III, J. L. Ingraham, E. C. C. Lin, K. B. Low, B. Magasanik, W. S. Reznikoff, M. Riley, M. Schaechter, and H. E. Umbarger (ed.), Escherichia coli and Salmonella: cellular and molecular biology, 2nd ed., vol. 1. American Society for Microbiology, Washington, D.C.

16. Jensen, K. F. 1993. The Escherichia coli K-12 "wild types" W3110 and MG1655 have an rph frameshift mutation that leads to pyrimidine starvation due to low pyrE expression levels. J. Bacteriol. 175:3401-3407. 
16a. Klauck, E., J. Bohringer, and R. Hengge-Aronis. 1997. The LysR-like regulator LeuO in Escherichia coli is involved in the translational regulation of $r p o S$ by affecting the expression of the small regulatory DsrA-RNA. Mol. Microbiol. 25:559-569.

17. Lange, R., D. Fischer, and R. Hengge-Aronis. 1995. Identification of transcriptional start sites and the role of ppGpp in the expression of $r p o S$, the structural gene for the $\sigma^{\mathrm{S}}$ subunit of RNA polymerase in Escherichia coli. J Bacteriol. 1995 Aug;177:4676-80.

18. Lange, R. and R. Hengge-Aronis. 1991. Identification of a central regulator of stationary-phase gene expression in Escherichia coli. Mol. Microbiol. 5:49-59.

19. Lange, R., and R. Hengge-Aronis. 1994. The cellular concentration of the $\sigma^{\mathrm{S}}$ subunit of RNA polymerase in Escherichia coli is controlled at levels of transcription, translation and protein stability. Genes Devel. 8:1600-1612.

20. Lease, R. A. and M. Belfort. 2000. Riboregulation by DsrA RNA: trans-actions for global economy. Mol. Microbiol. 38:667-672.

21. Loewen, P. C., B. Hu, J. Strutinsky, and R. Sparling. 1998. Regulation in the rpoS regulon of Escherichia coli. Can. J. Microbiol. 44:707-717.

22. Loewen, P. C., I. Von Ossowski, J. Switala, and M. R. Mulvey. 1993. KatF $\left(\sigma^{S}\right)$ synthesis in Escherichia coli is subject to posttranscriptional regulation. J. Bacteriol. 175:2150-2153.

23. Majdalani, N., C. Cunning, D. Sledjeski, T. Elliott and S. Gottesman. 1998. DsrA RNA regulates translation of RpoS message by an anti-antisense mechanism, independent of its action as an antisilencer of transcription. Proc. Natl. Acad. Sci. USA 95:12462-12467.

24. McCann, M. P., C. D. Fraley, and A. Matin. 1993. The putative sigma factor KatF is regulated posttranscriptionally during carbon starvation. J. Bacteriol. 175:2143-2149.

25. Metcalf, W. W., W. Jiang, L. L. Daniels, S. K. Kim, A. Haldimann, and B. L. Wanner. 1996. Conditionally replicative and conjugative plasmids carrying lacZ alpha for cloning, mutagenesis and allele replacement in bacteria. Plasmid 35:1-13.

26. Metzger, S., G. Schreiber, E. Aizenman, M. Cashel, and G. Glaser. 1989. Characterization of the relA1 mutation and a comparison of relA1 with new relA null alleles in Escherichia coli. J. Biol. Chem. 264:21146-21152.

27. Miller, J. H. 1972. Experiments in molecular genetics. Cold Spring Harbor Laboratory, Cold Spring Harbor, N.Y. 
28. Muffler, A., D. Fischer, and R. Hengge-Aronis. 1996. The RNA-binding protein HF-I, known as a host factor for phage $\mathrm{Q} \beta$ RNA replication, is essential for rpoS translation in Escherichia coli. Genes Devel. 10:1143-1151.

29. Mukhopadhyay, S., J. P. Audia, R. N. Roy, and H. E. Schellhorn. 2000. Transcriptional induction of the conserved alternative sigma factor RpoS in Escherichia coli is dependent on BarA, a probable two-component regulator. Mol. Microbiol. 37:371-381.

30. Mulvey, M. R., J. Switala, A. Borys, and P. C. Loewen. 1990. Regulation of transcription of katE and katF in Escherichia coli. J. Bacteriol. 172:6713-6720.

31. Pratt, L. A., and T. J. Silhavy. 1996. The response regulator SprE controls the stability of RpoS. Proc. Natl. Acad. Sci. USA 93:2488-2492.

32. Pratt, L. A. and T. J. Silhavy. 1998. Crl stimulates RpoS activity during stationary phase. Mol. Microbiol. 29:1225-1236.

33. Repoila, F. and S. Gottesman. 2001. Signal transduction cascade for regulation of RpoS: temperature regulation of DsrA. J. Bacteriol. 183:4012-4023.

34. Schellhorn, H. E. and V. L. Stones. 1992. Regulation of katF and katE in Escherichia coli K-12 by weak acids. J. Bacteriol. 174:4769-4776.

35. Schweder, T., K-H. Lee, O. Lomovskaya, and A. Matin. 1996. Regulation of Escherichia coli starvation sigma factor $\left(\sigma^{S}\right)$ by ClpXP protease. J. Bacteriol. 178:470-476.

36. Shiba, T., K. Tsutsumi, H. Yano, Y. Ihara, A. Kameda, K. Tanaka, H. Takahashi, M. Munekata, N. N. Rao, and A. Kornberg. 1997. Inorganic polyphosphate and the induction of rpoS expression. Proc. Natl. Acad. Sci. USA 94:11210-11215.

37. Silhavy, T. J., M. L. Berman, and L. W. Enquist. 1984. Experiments with gene fusions. Cold Spring Harbor Laboratory, Cold Spring Harbor, N. Y.

38. Simons, R. W., F. Houman, and N. Kleckner. 1987. Improved single and multicopy lac-based cloning vectors for protein and operon fusions. Gene 53:85-96.

39. Sledjeski, D. D., A. Gupta, and S. Gottesman. 1996. The small RNA, DsrA, is essential for the low temperature expression of RpoS during exponential growth in Escherichia coli. EMBO J. 15:3993-4000. 
40. Tsui, H. C., H. C. Leung, and M. E. Winkler. 1994. Characterization of broadly pleiotropic phenotypes caused by an $h f q$ insertion mutation in Escherichia coli. K-12. Mol. Microbiol. $13: 35-49$.

41. Webb, C., M. Moreno, M. Wilmes-Riesenberg, R. Curtiss III, and J. W. Foster. 1999. Effects of DksA and ClpP protease on sigma S production and virulence in Salmonella typhimurium. Mol. Microbiol. 34:112-123.

42. Xiao, H., M. Kalman, K. Ikehara, S. Zemel, G. Glaser, and M. Cashel. 1991. Residual guanosine 3', 5'-bispyrophosphate synthetic activity of relA null mutants can be eliminated by spoT null mutations. J. Biol. Chem. 266:5980-5990.

43. Yu, D., H. M. Ellis, E. C. Lee, N. A. Jenkins, N. G. Copeland, and D. L. Court. 2000. An efficient recombination system for chromosome engineering in Escherichia coli. Proc. Natl. Acad. Sci. USA 97:5978-5983.

44. Zhang, A., S. Altuvia, A. Tiwari, L. Argaman, R. Hengge-Aronis, and G. Storz. 1998. The OxyS regulatory RNA represses $r p o S$ translation and binds the Hfq (HF-I) protein. EMBO J. 17:6061-6068. 
Fis regulates transcriptional induction

of RpoS in Salmonella enterica

Matthew Hirsch

Thomas Elliott*

Current Address:

Department of Microbiology, Immunology and Cell Biology

West Virginia University

Morgantown, WV 26506

Corresponding author:

Thomas Elliott

West Virginia University Health Science Center

MICB, HSC-N

Morgantown, WV

telliott@hsc.wvu.edu

Phone: 304 293-2676

Fax: 304-293-7823 


\begin{abstract}
The sigma factor RpoS is known to regulate at least 60 genes in response to environmental sources of stress or during growth to stationary phase (SP). Accumulation of RpoS relies on integration of multiple genetic controls including regulation at the levels of transcription, translation, protein stability and protein activity. Growth to SP in rich medium results in a 50-fold induction of RpoS, although the mechanism of this regulation is not understood. Here we characterized the activity of promoters serving rpoS in Salmonella enterica, and report that regulation of transcription during growth into SP depends on Fis, a DNA-binding protein whose abundance is high during exponential growth and very low in SP. A fis mutant of S. enterica shows a nine-fold increase in expression from the major rpoS promoter $\left(\mathrm{P}_{r p o s}\right)$ during exponential growth, whereas expression during SP is unaffected. Increased transcription from $\mathrm{P}_{\text {rpos }}$ in the absence of Fis eliminates the 10-fold transcriptional induction as cells enter SP. The mutant phenotype can be complemented by wild type fis carried on a single-copy plasmid. Fis regulation of $r p o S$ requires the presence of a Fis site positioned at -50 with respect to $\mathrm{P}_{r p o S}$ and this site is bound by Fis in vitro. A model is presented in which Fis binding to this site allows repression of $r p o S$ specifically during exponential growth, thus mediating transcriptional regulation of rpos.
\end{abstract}




\section{Introduction}

Bacteria maintain intricate signaling networks that sense the environment and adjust cellular physiology accordingly. In Salmonella enterica (S. enterica) and Escherichia coli (E. coli), less favorable growth conditions (e.g. nutrient limitation, starvation, low temperature, or osmotic shock) initiate a general stress response by triggering the synthesis of the RNA polymerase sigma factor RpoS $\left(\sigma^{\mathrm{S}}\right)$. By directing RNA polymerase to promoters of specific genes involved in stress resistance, RpoS serves as the central regulator of the general protective response, also known as SP, and thus increases survival (28). The importance of RpoS to S. enterica pathogenesis is evident from a mouse model involving lethal infections where rpoS mutants are completely avirulent (22).

The complexity of RpoS regulation is illustrated by the variety of mechanisms reported so far in E. coli: transcription, translation, protein turnover and protein activity (28). One of the best characterized induction phenomena is regulation of RpoS translation at low temperature in rich medium $\left(\leq 30^{\circ} \mathrm{C}\right)$. This stimulus increases transcription of a regulatory RNA, DsrA, which can pair with an upstream antisense element in the leader region of the rpoS transcript to relieve the antisense element's inhibition of $r p o S$ translation (38). This process requires the Sm-like RNA-binding protein, Hfq, and results in activation of RpoS expression at a post-transcriptional step. Notably, $h f q$ mutants show normal SP induction of RpoS in rich medium, both in E. coli (29) and S. enterica (our unpublished data).

Another regulatory pathway limiting RpoS abundance in growing cells is proteolytic degradation involving the ATP-dependent ClpXP protease and a response regulator called MviA (in S. enterica) or 
SprE / RssB in E. coli $(52,62)$. In this pathway MviA is activated by an unknown stimulus through phosphorylation on D58, which substantially increases its ability to bind to RpoS. The relevant kinase has not yet been found $(16,28)$. The binding event (dependent on K173 of RpoS) results in a sequestered non-functional RpoS molecule and thus can modulate RpoS activity in itself $(8,75)$. The MviA-RpoS complex also interacts with the ClpXP protease, which then actively degrades RpoS, recycling MviA (44, 52). RpoS elevates transcription of the response regulator during SP, thus constituting an auto-regulatory loop in which the concentration of MviA is a limiting factor for the rate of RpoS degradation in vivo $(53,57)$.

Perhaps the most striking induction of RpoS is observed during growth to SP in rich medium, where the level of induction exceeds 30-fold, based on the activity of RpoS-responsive reporters and rpoS-lac fusions (28, our unpublished data). The transcriptional component of this induction ranges from 5-10 fold $(28,29)$. Expression levels are significantly lower in the absence of guanosine tetraphosphate during both growth and SP, but the actual induction ratio is nearly unchanged (29). The cyclic-AMP (cAMP) receptor protein, Crp, is also thought to be involved in rpoS transcriptional control yet the effect of the mutants is modest and interpretation is difficult due to the growth deficiency of the crp or cya (defective in adenylate cyclase) mutants in combination with growth-rate transcriptional control of rpoS $(36,37)$.

In this study we show that the Fis protein (factor for inversion stimulation) is involved in RpoS regulation during growth in rich medium. Fis is a DNA-binding and bending protein that was initially characterized for its stimulatory role in site-specific DNA recombination $(31,34)$. Fis has been implicated in many other processes such as stimulation of excision and integration of lambda $(4,5,20$, 
67), DNA replication at oriC $(24,26,58)$, transposition (70), invasion of HEp-2 cells (71) and transcriptional activation and repression of several genes including hns, leuV, gyr, tyrT, proP, nuo, osmE, and rRNA operons $(21,56,33,3,72,68,12,74)$.

Here we investigate $r p o S$ promoter activity and demonstrate that Fis mutants have elevated expression of rpoS that is specific to exponential phase. This pattern of regulation is in good agreement with the known variation in Fis abundance in different phases of growth: Fis is undetectable in SP but is present at over 40,000 dimers per cell upon dilution into fresh medium $(2,6,50)$. Based on these results and the requirement for specific sequences upstream of the major rpoS promoter, we present an intuitive model for transcriptional regulation of rpoS in which Fis binds to and represses transcription from $\mathrm{P}_{\text {rpos. }}$.

\section{Materials and Methods}

\section{Media and growth conditions.}

Bacteria were grown at $37^{\circ} \mathrm{C}$ (except where noted) in various media: $\mathrm{LB}$ medium (63), LB medium containing 1x NCE minimal salts (buffered LB medium; 10), nutrient broth supplemented with $5 \mathrm{~g} /$ liter of $\mathrm{NaCl}$ (NB, Difco), and brain heart infusion (BHI, Difco). Liquid minimal medium was morpholinepropanesulfonic acid (MOPS) medium (48) as modified (11), supplemented with $0.2 \%$ glucose as the carbon and energy source. When indicated, minimal medium was supplemented with $1 \%$ casamino acids (CAA, Difco). Plates were prepared using nutrient agar (Difco). Antibiotics were added to final concentrations in selective media as follows: $100 \mu \mathrm{g}$ of sodium ampicillin $/ \mathrm{ml}, 20 \mu \mathrm{g}$ of 
chloramphenicol $/ \mathrm{ml}, 50 \mu \mathrm{g}$ of kanamycin sulfate $/ \mathrm{ml}$, and $20 \mu \mathrm{g}$ of tetracycline hydrochloride $/ \mathrm{ml}$, except that the ampicillin concentration was reduced to $50 \mu \mathrm{g} / \mathrm{ml}$ for use with single copy plasmids.

MacConkey lactose agar was prepared as described (43). X-Gal (5-bromo-4-chloro-3-indolyl- $\beta$-Dgalactopyranoside) was used at $50 \mu \mathrm{g} / \mathrm{ml}$.

Bacterial strains and construction. Most of the strains used in this study were derived from wild type Salmonella enterica LT2 (serovar Typhimurium); all strains are described in Table 8. We obtained strain LT2 from John Roth. Although this strain is the reference wild type, it has been shown to contain a nonfunctional mviA gene (V102G substitution) and is therefore defective in regulated RpoS turnover by ClpXP proteolysis $(9,16)$. LT2A is a derivative of LT2 whose only known difference is that it contains a functional mviA gene. LT2A was used to investigate RpoS proteolysis as indicated in the text (16). The phage P22 mutant HT105/1 int-201 was used for transduction in S. enterica by standard methods (18), while P1 vir was used for transduction of fusions carried on the E. coli chromosome into S. enterica strain TE7304 (see below). Transductants inheriting cya::Tn10 and crp::Tn10 insertions were selected on NB plates containing tetracycline and supplemented with $0.2 \%$ glucose. The $c r p *$ allele used in this study was originally isolated by Ailion et al. (1).

Construction of promoter fusions. The system used to construct promoter fusions relies on the cloning of PCR products amplified from S. enterica LT2 chromosomal DNA. In the Genbank sequence file AE008833.1 (40), the complement of the $n l p D$ and rpoS sequences is given. Here, positions are indicated using coordinates from AE008833.1, including nlpD (bp 13178 -> 12045) and rpoS (bp 11982 $>10990$ ), and coordinates are listed with the same polarity as the genes (higher to lower numbers). 
DNA fragments were produced with flanking EcoRI (upstream) and BamHI (downstream) sites and cloned into pRS551 to generate transcriptional lacZ fusions $(19,64)$. To this end we made four different

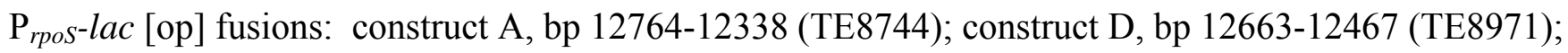
construct E, bp 12663-12528 (TE8868); and construct F, bp 12586-12467 (TE8947). (All primer sequences are available upon request.) The $\mathrm{P}_{n l p D}$-lac [op] fusion was generated in the same manner and contains the region encompassing bp 13471-13052. Recombinant plasmids were used to transform DH5 $\alpha$ and the fusions confirmed by PCR and DNA sequencing. The fusions were recombined into the E. coli chromosome as described (19), then transduced into S. enterica using P1 vir. In S. enterica, they are located at the put locus in single copy.

Construction of deletion/insertions and point mutations. Most other constructs were made by direct transformation of S. enterica with different DNA segments amplified by PCR, utilizing the lambda red recombination system as provided on plasmid pKD46 (17). Exponential-phase recipient cells, growing at $30^{\circ} \mathrm{C}$ with selection for $\mathrm{Amp}^{\mathrm{R}}$, were induced by treatment with $0.2 \%$ arabinose for $1 \mathrm{hr}$ before electroporation, after which transformants were plated and selected at $37^{\circ} \mathrm{C}$.

To construct site-directed mutations in $\mathrm{P}_{r p o s}-$ lac [op], we developed a multi-step method. In order to prevent unwanted recombination events, $\mathrm{C} \mathrm{Cam}^{\mathrm{R}}$ cassette was inserted at the native rpoS locus (deleting bp 12720-12142). The $\mathrm{Cam}^{\mathrm{R}}$ cassette was amplified using rpoS-specific primers extended to provide homology to cat at the 3' end as follows: TGCTTTTGCCGTTACGCACCAC (upstream) and GCCTCAGGCATTTGAGAAGCAC (downstream). The resulting insertion deleted the rpoS promoter region and gave loss of $r p o S$ function as determined by absence of visible catalase activity (production of bubbles after spotting $5 \mu l$ of hydrogen peroxide on a patch of bacteria). In the second step, a tetAR 
cassette (pWM7 as the template; 42) was inserted to delete the rpoS promoter of the $\mathrm{P}_{\text {rpos }}$-lac [op] fusion. Amplification used rpoS-specific primers extended to provide homology to tet at the 3' end as follows: CTCTTGGGTTATCAAGAGGG (tetA); ACTCGACATCTTGGTTACCG (tetR). The tetAR

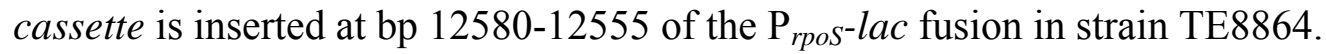

In the third step, a point mutation and an in-frame deletion mutation (both unmarked by drug resistance) were introduced by transformation of strain TE8864. PCR products were prepared containing either three point mutations or an in-frame deletion of a predicted Fis binding site. The upstream (mutagenic) primers used were GACCAGGTCTGCACㅌAATT and CACCCAGGCGGATGCAGCACAGCAAGGAGTTGTGACCAGG- $\Delta$ -

GCAGTTGCGTCTCAACCAAC, together with a downstream primer from within lacZ. The desired transformants acquired the Fis site mutation and lost the tet $A R$ insertion. These transformants also acquired a functional rpoS promoter; this allowed screening for them $\mathrm{as} \mathrm{Lac}^{+} \mathrm{Tet}^{\mathrm{S}}$ colonies that were confirmed by sequencing.

\section{Isolation of rpoS::MudJ insertion and construction of promoter deletions. A large pool of}

MudJ insertions in LT2 was first generated by standard methods (30). A phage P22 lysate grown on this pool was then used to transduce strain TE8607 to $\mathrm{Cys}^{+} \mathrm{Kan}^{\mathrm{R}}$ on minimal medium containing X-gal. Blue $\left(\mathrm{Lac}^{+}\right)$transductants were then screened by testing patches of cells grown on NB Kan agar for catalase activity as described above. Putative $r p o S$ mutants were purified and tested by PCR, then confirmed by DNA sequencing. The rpoS1082::MudJ insertion used in these experiments lies at codon 66 of $r p o S$ (bp 11784; strain TE8737). 
Several insertion / deletions were constructed in the TE8737 background using the tetAR cassette and the lambda red method. This method utilized strain TE8738, rpoS::MudJ containing pKD46 (17), as the target for transformation with PCR-generated tetAR inserts derived from pWM7. The linked MudJ and tetAR insertions were then backcrossed by P22 mediated transduction to S. enterica LT2 and the insertion joint was confirmed by DNA sequencing (see Table 8 for exact location of the tetAR insertions). To construct an in-frame deletion of the $r p o S$ promoter region, we inserted tetAR to knock out $\mathrm{P}_{r p o s}$ in the context of the MudJ fusion (TE8913) and subsequently transformed the strain with pKD46 (TE8915). This strain served as the recipient for transformation with a PCR product carrying an in-frame deletion of the rpoS promoter (bp12582-12540). The deletion was generated by PCR using a 60-mer oligonucleotide with rpoS sequence interrupted by a 30 bp deletion including both the -35 and 10 hexamers of the rpoS promoter:

\section{AGGAGTTGTGACCAGGTCTGCACAAAATTCCACCGTTGCA- $\Delta-$}

GAGGGCTCAGGTGAACAAAG, together with a downstream primer at bp 12301. Although deleted for the $\mathrm{P}_{r p o s}$ promoter, lac is expressed from $\mathrm{P}_{n l p D}$ in these strains, hence, transformants were screened for a subtle $\mathrm{Lac}^{+}$phenotype and confirmed as $\mathrm{Tet}^{\mathrm{S}}$ and by DNA sequencing.

5'RACE reaction. RNA was isolated from wild type LT2 cells during exponential phase using a RNAeasy mini Kit (Qiagen). RNA served as the template for the 5' RACE reactions performed using the BD SMART RACE cDNA amplification kit as described by the manufacturer (BD Biosciences) with one exception. A rpoS specific primer was used in first strand cDNA synthesis, positioned at the initiation codon of rpoS. RACE reaction products were eluted from $1 \%$ agarose gels, using the NucleoTrap Gel extraction kit (BD Biosciences), T/A cloned into a pCR4-TOPO vector (Invitrogen) and subsequently used to transform TOP 10 Electrocomp cells (Invitrogen). Transformants were selected on 
NB Kan plates containing X-gal (screening for a Lac phenotype). Plasmids were analyzed by PCR amplification of the insert followed by DNA sequencing.

Assay of $\beta$-galactosidase. Cells were centrifuged, resuspended in Z-buffer $(100 \mathrm{mM} \mathrm{NaPO} 4[\mathrm{pH}$ 7.0], $10 \mathrm{mM} \mathrm{KCl}, 1 \mathrm{mM} \mathrm{MgSO}_{4}$ ) and then permeabilized by treatment with sodium dodecyl sulfate (SDS) and chloroform (43). The samples from exponential-phase time points were concentrated before assay to be approximately equal in density to samples obtained at later times. For all experiments, exponential phase is defined as $\mathrm{OD}_{600}=0.25$. Assays were performed in Z-buffer containing $50 \mathrm{mM} \beta$ mercaptoethanol by a kinetic method using a plate reader (Molecular Dynamics). Activities (change in optical density at $420 \mathrm{~nm}\left[\mathrm{OD}_{420}\right]$ per minute) were normalized to actual cell density $\left(\mathrm{OD}_{650}\right)$ and were always compared to appropriate controls assayed at the same time. All the $\beta$-galactosidase assays were performed within 1 hour of the time of sampling, during this interval cultures were kept on ice in $Z$ buffer. The values shown are averages of at least 3 experiments with a standard deviation of less than $15 \%$ unless otherwise stated.

Immunological detection of proteins. For Western blots, cultures were grown as described in the text. Electrophoresis and protein transfer were as described previously $(13,16)$. After transfer to a Sequi-Blot PVDF membrane (BioRad), blots were blocked in non-fat milk and incubated in Phosphate Buffered Saline-Tween containing the anti-RpoS monoclonal antibody R12 (13), which is of the $\gamma 2 \mathrm{a}$ isotype. After $30 \mathrm{~min}$. incubation, blots were washed twice in PBS-Tween, then incubated for $30 \mathrm{~min}$ in PBS-Tween containing biotinylated goat anti-mouse immunoglobin (Ig), and finally with streptavidinconjugated horseradish peroxidase (both from Southern Biotechnology Associates). Detection was by 
enhanced chemiluminescence (Amersham Biosciences).

Complementation test. TE8905 harbors pFis349 (71), which contains a $1.68 \mathrm{~kb}$ DNA fragment encoding the orf1-fis operon of S. enterica cloned into the single-copy plasmid pGS349 (32). Plasmid DNA isolated from an E. coli host was used to transform an $\mathrm{rm}^{+}$S. enterica strain (TE315) and subsequently, wild type (TE8744) and $\Delta$ fis (TE8764) strains containing the $\mathrm{P}_{r p o s}$-lac [op] fusion. Cultures were grown in LB with ampicillin and assayed for $\beta$-galactosidase as described in text.

Gel-Shift assay. DNA target fragments were amplified from the $S$ enterica chromosome using primers engineered to generate EcoRI and BamHI restriction sites at opposite ends of the product. We made three different shift targets, designated A, B, and C, that correspond, respectively, to bp 12750-12604, bp 12643-12547, and bp 12574-12475 of the $\mathrm{P}_{\text {rpos }}$ promoter. We also generated two mutant targets derived from target $\mathrm{B}$, designated $\mathrm{B}^{*}$ and $\mathrm{B}^{\Delta}$, using the same primers as for $\mathrm{B}$ but chromosomal templates isolated from strains TE8887 and TE8895. The PCR reactions were purified (Qiagen) and digested with EcoRI and BamHI restriction endonucleases. The fragment ends were then labeled by incorporation of $\left[\alpha^{32} \mathrm{P}\right] \mathrm{ATP}$ using the Klenow fragment of DNA polymerase as described by the manufacturer (Promega), and purified using a PCR purification kit. Radiolabeled DNA fragments (ca. $15,000 \mathrm{cpm}$ per reaction) were incubated with purified Fis protein (gift of R. Johnson), for $15 \mathrm{~min}$ at room temperature in buffer containing $20 \mathrm{mM}$ Tris $\mathrm{HCl}[\mathrm{pH}$ 7.5], $80 \mathrm{mM} \mathrm{NaCl}, 1 \mathrm{mM}$ EDTA, 5\% glycerol, and $2 \mathrm{ng}$ of poly[d(I-C)]/ $\mu$ l. Binding reaction products were analyzed by electrophorhesis on a $8 \%$ native polyacrylamide gel as previously described (72). The gels were then dried and the radioactive DNA detected by autoradiography. 
A.

\begin{tabular}{lcccc}
\hline & & \multicolumn{3}{c}{$\beta$-galactosidase activity } \\
\cline { 3 - 5 } Reporter & Background & $\mathrm{E}$ & $\mathrm{SP}$ & $\mathrm{SP} / \mathrm{E}$ \\
\hline \multirow{2}{*}{ katE-lac[op]} & $w t$ & 0.41 & 17 & 41 \\
& $c l p X$ & 1.9 & 75 & 39 \\
& $m v i A$ & 2.4 & 75 & 31
\end{tabular}

B.

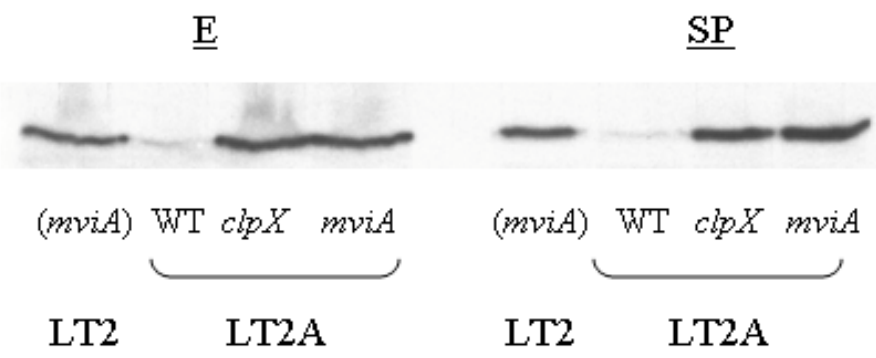

Figure 9. SP induction of RpoS is normal in turnover-defective mutants.

(A) LT2A strains harboring the RpoS reporter, katE-lac [op], and carrying the indicated mutations were sampled for $\beta$-galactosidase activity during exponential phase at $\mathrm{OD}_{600}=0.25(\mathrm{E})$, and after 24 hours of growth (SP). SP induction was calculated as the ratio of the SP activity to the exponential phase value (SP/E). (B) The indicated strains were probed for RpoS protein at time points $\mathrm{E}$ and SP by Western analysis. The gel for the exponential experiment was loaded with the lysate recovered from 10-fold more cells in order to visualize low concentrations of RpoS in the LT2A background. All strains were grown at $37^{\circ} \mathrm{C}$ in LB medium. Strains: LT2A mviA (TE6851); LT2A clpX (TE6850).

\section{Results}

\section{SP induction of RpoS in S. enterica is normal even in the absence of regulated proteolysis.}

Studies of Schweder (62) and Zgurskaya (73) suggest that in starving E. coli cells RpoS abundance increases mainly as a result of increased protein stability. Another E. coli study reports RpoS induction in the absence of an intact RpoS degradation pathway (52). We investigated the role of this pathway in the induction of RpoS for S. enterica grown to SP in rich medium (LB). A katE-lac [op] fusion was used as a reporter of RpoS activity $(13,45)$. Expression of the katE-lac fusion was measured both in a 
wild type LT2A background and in clpX and mviA mutants defective in regulated turnover of RpoS. [The mviA gene is the S. enterica ortholog of E. coli rssB / sprE (9)].

During exponential growth $\left(\mathrm{OD}_{600}=0.25\right)$, expression of katE-lac in both the clpX and mviA mutants was approximately five-fold higher than in the wild type (Fig. 9A). This result is consistent with the idea that MviA and ClpXP function together to degrade RpoS during exponential growth (16, 44, 52). A quantitatively similar increase in katE-lac expression in the mutant backgrounds was also observed after 24 hours of growth (defined as SP). This result suggests that the MviA and ClpXP pathway for RpoS degradation functions at a similar level during both exponential growth and at SP in LB medium. The normal SP induction ratio for katE-lac expression in the mviA and clpX mutants indicates that this proteolytic pathway does not regulate SP induction of RpoS (ratio shown as SP/E, Fig.9A). Somewhat higher katE-lac activity in the mviA mutant compared to the $\operatorname{clpX}$ mutant is consistent with a role for MviA to sequester RpoS even if proteolysis is blocked $(8,75)$.

To confirm this result, the abundance of RpoS was determined by Western blot analysis of cells in both exponential growth and SP (10-fold more material was loaded for exponential cells to allow visualization of RpoS in wild type). Both $c l p X$ and mviA mutations resulted in a marked increase in RpoS abundance compared to wild type LT2A. The increased amount of RpoS in the mutants appeared similar to the amount of RpoS detected in the LT2 strain, which is naturally defective in mviA function. The relative increase in RpoS observed in the mutant backgrounds for cells in exponential growth is apparently the same as during SP (Fig.9B). The combined lac fusion and Western blot results indicate that in S. enterica, the MviA/ClpXP turnover pathway does not mediate the SP induction of RpoS in LB medium. 
Promoters contributing to rpoS expression in S. enterica. We next considered transcriptional regulation of rpoS. Studies in E. coli $(28,29,60)$ indicate that regulation of $r p o S$ at the transcriptional level may be particularly important when cells grow to SP in rich medium, in contrast to the posttranscriptional mechanisms mediating response to osmotic shock, carbon starvation or low temperature. We previously demonstrated a 15-fold induction of an rpoS-lac transcriptional fusion, when the rpoS gene was derived from E. coli, and studied both in an E. coli (29) and an S. enterica host (13).

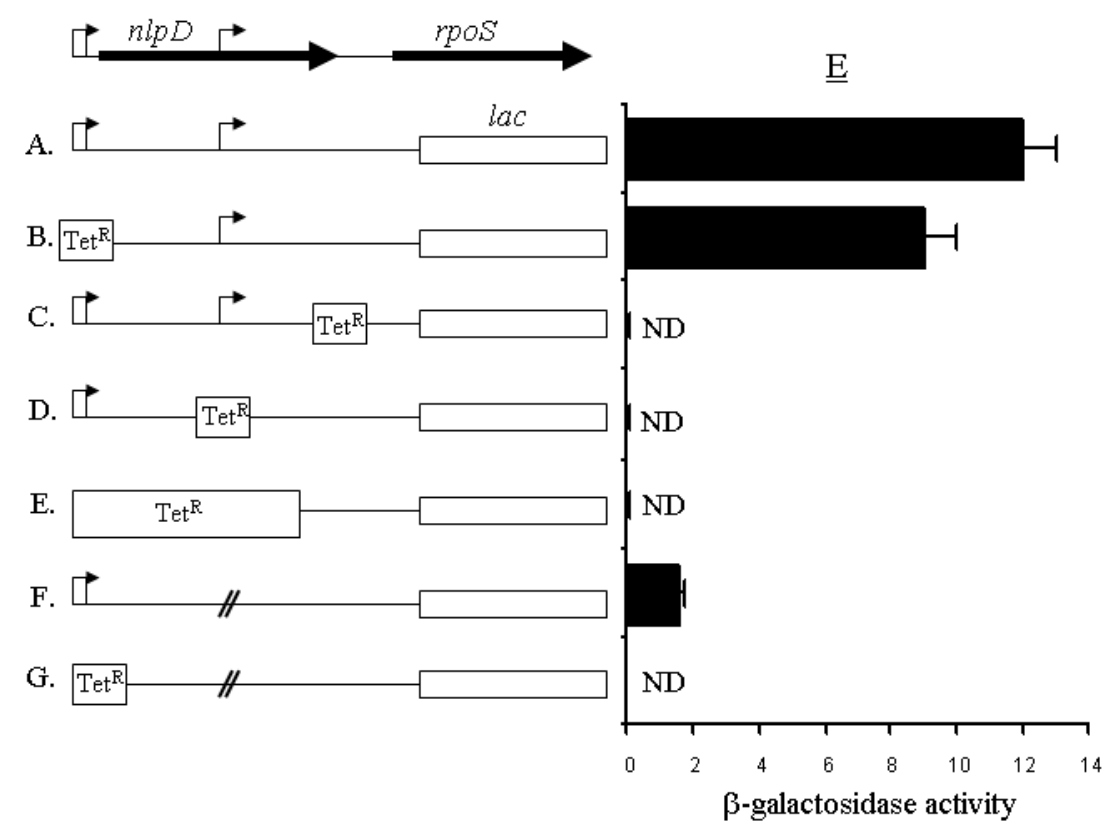

Figure 10. Promoter activitv analvzed using rpoS::MudJ as a reporter.

The top line depicts the genetic organization of the rpoS region, with long horizontal arrows showing gene and transcriptional polarity, and small bent arrows indicating the promoters contributing to rpoS expression. Construct A contains the lac fusion formed by MudJ insertion at codon 66 of rpoS, and is otherwise wild type. Constructs B to E contain the same rpoS::MudJ insertion as construct A and in addition, contain insertions of a tetracycline resistance cassette $\left(\mathrm{Tet}^{\mathrm{R}}\right)$ accompanied by deletions (insertion / deletions). Construct $\mathrm{F}$ is identical to construct $\mathrm{A}$ except it contains an in-frame deletion (represented by slanted lines) of the $\mathrm{P}_{\text {rpos }}$ promoter. Construct $\mathrm{G}$ is like construct $\mathrm{F}$ but also contains the insertion / deletion from construct $\mathrm{B}$. The MudJ element is not drawn to scale. Construction details are given in Materials and Methods and precise insertion sites are given in Table 8. Shown next to each fusion is the $\beta$-galactosidase activity as determined at $\mathrm{OD}_{600}=0.25$ (E) in cultures grown at $37^{\circ} \mathrm{C}$ in rich medium. [N.D.(not detected).] Strain numbers for these constructs are as follows: A, TE8737; B, TE8901; C, TE8907; D, TE8913; E, TE8914; F, TE8925; and G, TE8937. 
Analysis of rpoS transcription in E. coli by primer extension, and in Salmonella dublin by both primer extension and Northern blot, established that rpoS is transcribed from two distinct promoter regions (35, $51,66)$. Two closely-spaced and relatively weak promoters $\left(\mathrm{P}_{n l p D}\right)$ generate a bicistronic $n l p D-r p o S$ message, while the major promoter $\left(\mathrm{P}_{r p o s}\right)$ is located approximately in the center of the $n l p D$ gene and generates a monocistronic rpoS transcript with a long untranslated leader region of 566 nucleotides (35, 51). E. coli and $S$. dublin share identical -35 and -10 hexamers for $P_{r p o s}$ with a 17 bp spacer, and initiate transcription at the same nucleotide. This region of S. enterica is identical to S. dublin and therefore it is likely that the defined $\mathrm{P}_{\text {rpos }}$ promoter of E. coli and S. dublin is conserved in S. enterica. We investigated the transcriptional start of $\mathrm{P}_{r p o s}$ in $S$. enterica using RACE cDNA amplification. Total cellular RNA harvested during exponential phase served as template in a reverse transcription reaction that exhibits terminal transferase activity, adding 3-5 residues to the 3' end of the first strand cDNA. These residues anneal to an oligonucleotide that serves as an extended template for reverse transcriptase thus generating a complete cDNA copy of the original RNA with known sequence at the end. The first strand cDNA is then used directly in a 5' RACE PCR reaction to generate double stranded cDNA products. Three cDNA products were observed using a primer positioned at the initation codon of $r p o S$ and these corresponded to the predicted sizes of transcripts from $\mathrm{P}_{n l p D}$ and $\mathrm{P}_{r p o s}$ (data not shown). The product representing $\mathrm{P}_{r p o s}$ was cloned and sequenced. The results positioned the first base of the transcript 566 nucleotides upstream of the rpoS coding region at the identical initiating nucleotide of $\mathrm{P}_{\text {rpos }}$ in E. coli and S. dublin.

Genetic analysis of the region upstream of rpoS in S. enterica suggests a similar pattern of transcriptional control compared to S. dublin and E. coli. We first isolated an insertion of the lac fusionforming transposon MudJ in the rpoS gene, forming a transcriptional fusion of $r p o S$ to lac (at codon 66 
of $r p o S$ ), to use as a reporter of in vivo transcriptional regulation (Fig. 10). Expression of the rpoS::MudJ fusion was analyzed during exponential phase in combination with insertion / deletion mutations constructed with a tet cassette predicted to affect the promoters serving rpoS or a deletion mutation of $\mathrm{P}_{r p o s}$ (Fig. 10). All constructs were placed at the native rpoS locus in the bacterial chromosome.

Insertion of tet downstream of $\mathrm{P}_{r p o s}$ or an insertion of tet which also makes a small deletion encompassing $\mathrm{P}_{r p o s}$, eliminated detectable activity of the rpoS::MudJ reporter (Fig. 10, constructs $\mathrm{C}$ and $\mathrm{D}$, lower limit of detection is $0.8 \mathrm{U}$ ). In contrast, an insertion / deletion of the $\mathrm{P}_{n l p D}$ promoter region but retaining $\mathrm{P}_{r p o S}$ showed relatively high $(\approx 75 \%)$ expression of the parental rpoS::MudJ (construct $\mathrm{B}$ ). In a further test, a precise in-frame deletion of $30 \mathrm{bp}$ including the conserved -35 and -10 hexamers of the $\mathrm{P}_{\text {rpos }}$ promoter was constructed (Fig. 10, construct F). This deletion reduces expression of $r p o S:$ MudJ to $\approx 15 \%$ of wild type. Similar to rpoS transcription in E. coli, we conclude that in S. enterica, $\mathrm{P}_{r p o s}$ is the major $r p o S$ promoter and $\mathrm{P}_{n l p D}$ plays a minor role.

Activity of the major $r p o S$ promoter, $\mathbf{P}_{\text {rpos }}$. Since most transcription of $r p o S$ originates from $\mathrm{P}_{r p o S}$, we characterized this promoter in isolation by using a lac fusion system described previously (19), in which the fusion is transferred to the S. enterica chromosome at the put locus. The fusion employed, $\mathrm{P}_{\text {rpos }}$-lac [op] (strain TE8744), includes 426 bp encompassing $\mathrm{P}_{\text {rpos }}$, from -209 to +217 with respect to the transcriptional start site. Activity of $\beta$-galactosidase was determined during exponential growth and in SP for cultures grown at $37^{\circ} \mathrm{C}$ in different media (Table 7). Expression of the $\mathrm{P}_{\text {rpos }}-$ lac [op] fusion increased 8-fold in SP during growth in two different rich media, which is consistent with results obtained from E. coli (28). 
Table 7. Activity of $\mathbf{P}_{r p o s}$ in different media

\begin{tabular}{llll}
\hline \multirow{2}{*}{ Growth Medium } & \multicolumn{3}{c}{$\beta$-galactosidase activity } \\
\cline { 2 - 4 } & $\mathrm{E}$ & SP & SP/E \\
\hline LB & 31 & 230 & 7.6 \\
Buffered LB & 28 & 262 & 9.3 \\
Buffered LB + glucose & 28 & 208 & 7.4 \\
BHI & 31 & 232 & 7.4 \\
Minimal glucose & 124 & 191 & 1.5 \\
Minimal glucose + casamino acids & 109 & 203 & 1.8 \\
\hline \multicolumn{4}{c}{${ }^{a}$ exponential phase (E) and stationary phase (SP) are defined } \\
in text. Values are averages with a variation of $<17 \%$. \\
Stationary phase induction is defined as SP/E.
\end{tabular}

Both carbon availability and the production of weak acids have been shown to affect rpoS transcription in E. coli $(37,46,60)$, and we hypothesized that these stimuli might be involved in SP induction of S. enterica rpoS. However, when activity and SP induction of $\mathrm{P}_{r p o s}$-lac [op] were assayed in buffered LB medium and buffered LB supplemented with $0.2 \%$ glucose the results were very similar to those observed in $\mathrm{LB}$ and brain heart infusion (BHI) medium. This suggests that neither $\mathrm{pH}$ changes nor lack of a suitable carbon source are responsible for SP induction. When strain TE8744 was grown in minimal medium (either with or without casamino acids), rpoS-lac expression increased approximately 3.5-fold over expression in LB. This increase in expression was specific to exponential growth in minimal medium. As a result, SP induction of rpoS transcription was much reduced. This result might be explained by growth-rate regulation of $r p o S$ transcription, however, our previous analysis of rpoS growth rate regulation in S. enterica indicated that it is mainly at a post-transcriptional level (16).

No auto-transcriptional role of $\boldsymbol{r p o S}$. The transcriptional start of $\mathrm{P}_{r p o s}$ is preceded by a typical 
sigma 70 RNA polymerase-dependent promoter consensus sequence (TTGCGT-17 nt spacerTATTCT). To examine whether RpoS contributes to its own transcription, we investigated the activity of several promoters as well as $\mathrm{P}_{\text {rpos }}$ in both wild type and rpoS mutant backgrounds. Strains harboring $\mathrm{P}_{r p o s}$-lac [op] or a $\mathrm{P}_{n l p D}$-lac [op] fusion, 419 nucleotides encompassing the $n l p D$ promoter region (-264 to +154 ), in either a wild type or rpoS background, were grown at $37^{\circ} \mathrm{C}$ in $\mathrm{LB}$ medium for 24 hours and assayed for $\tilde{\beta}$-galactosidase activity (Fig. 11). A small increase in the expression of $\mathrm{P}_{n l p D}$ and $\mathrm{P}_{\text {rpos }}$ was evident in the mutant background, rather than the decrease predicted by a model involving selftranscription. As a positive control, we used the katE-lac [op] fusion and observed a 95\% reduction in katE-lac activity in the rpoS mutant $(13,59)$. A lacUV5-lac [op] fusion was used as a negative control. Expression of this fusion demonstrated a $15-20 \%$ increase in the absence of RpoS, similar to that seen with the $\mathrm{P}_{n l p D}$ and $\mathrm{P}_{r p o s}$ fusions. These results confirm that RpoS is not involved in autoregulation during SP. The increased expression of sigma 70 promoters in the absence of RpoS is consistent with competition of sigma factors for RNA polymerase (23).

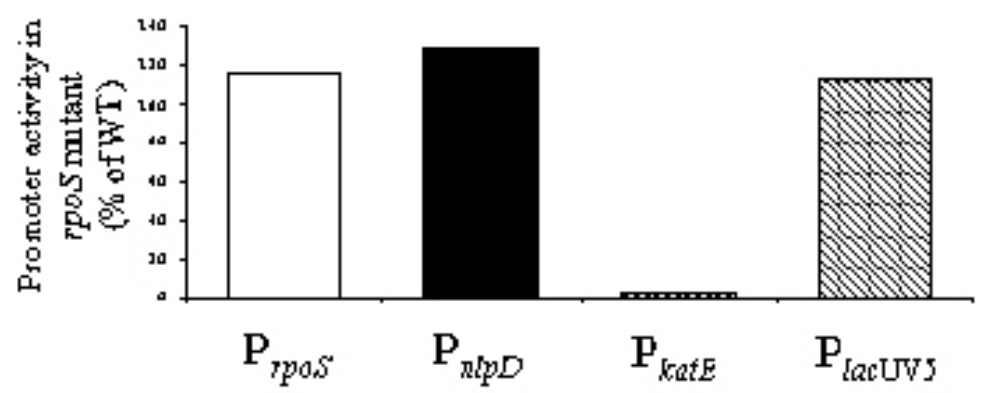

Figure 11. Testing autotranscription of rpoS.

LT2 strains harboring transcriptional lacZ fusions, expressed from the indicated promoters, in either a wild type or $r p o S$ mutant background, were grown at $37^{\circ} \mathrm{C}$ in LB medium for 24 hours and $\beta$-galactosidase activity was determined. Promoter activity in the rpoS mutant is plotted as a percentage of the activity in the corresponding wild type strain. Strains were as follows: $\mathrm{P}_{r p o s}$ (TE8744, TE8758), $\mathrm{P}_{n l p D}(\mathrm{TE} 8698, \mathrm{TE} 8761), \mathrm{P}_{\text {katE }}$ (TE6153, TE8760), $\mathrm{P}_{\text {lacuvs }}$ (TE6676, TE8759). 
A.

-175 GTATAGCCTGAATGTAGGGCAAACTTTGCAAGTTGGTAACGCATCCGGTA

-125 CGCCAATTACTGGCGGAAATGCGATCACCCAGGCGGATGCAGCACAGCAA

-7508iGAGTTGTGACCAGGTCTGCACAAAATTCCACCGTTGCAGTTGCGTCTCA

-25 ACCAACAATACGTATTCTGAGGGCTCAGGTGAACAAAGTGCTAACAAAA

+26 TGTTGCCAAACAACAAGCCTGCTGGGACGGTTGTCACA

B.

Fis binding consensus

Fis site Position

I. $\quad-173$ to -153

II. -117 to -97

III. -60 to -40

IV. $\quad+4$ to +24

V. +14 to 34

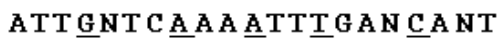

Sequence

Bit score

ATA $\underline{\mathrm{G} C \mathrm{CT}} \mathrm{AATGTAGGG} \underline{\mathrm{CA} A \mathrm{~A}} \quad 5.9$

$\mathrm{ACT} \underline{\mathrm{G} G C G A} \underline{\mathrm{A} T G C G A T C} \mathrm{ACC} \quad 6.1$

TCT G C A $\underline{\mathrm{A} A \mathrm{~A}} \underline{\mathrm{ATC}} \mathrm{TCC} \underline{\mathrm{C}} \mathrm{GTT} \quad 10.9$

GGT $\underline{G A C} \underline{A A A G G C T A A \underline{A A A}} \quad 4.1$

T G CTAAC $\underline{A} A \underline{A} \underline{A}$ T TT G C $\underline{C A A}$

Figure 12. Analysis of the sequence near $\mathbf{P}_{\text {rpos }}$.

(A) The sequence of the $\mathrm{P}_{\text {rpos }}$ promoter region is shown (bp 12764-12338 of AE008833.1). Predicted Fis binding sites are underlined, and arrows designate putative CRP-binding half-sites. The numbering is relative to the transcriptional start site (labeled +1 ). (B) The consensus sequence for Fis protein binding is given as well as the sequences of predicted Fis binding sites near $\mathrm{P}_{r p o s}$, individually designated by Roman numerals. The column labeled bit score represents the similarity of each putative site to a collection of known Fis binding sites as determined by information analysis (described in the text). Five of the most conserved bp in the consensus are marked with an underline (where present in each sequence), and the asterisk marks the axis of rotational symmetry for the Fis consensus sequence.

Computational analysis of the rpoS promoter. To further explore the transcriptional regulation of rpoS, we used the DNA-motif search engine available at http://arep.med.harvard.edu/ecoli_matrices/ to recognize potential protein binding sites in the region of the $\mathrm{P}_{r p o s}$ promoter (Fig. 12; 55). This program utilizes the known, characterized binding sites of 59 transcriptional regulators to predict putative binding 
sites throughout the entire E. coli chromosome. Results suggested putative binding sites for a large number of regulators including CytR, CRP, DnaA, FarR, Fis, FNR, HNS, IHF, GlpR, Lrp, MalT, MetJ, MetR, NarL, OmpR, SoxS and TyrR. Of these, only CRP has been reported as a regulator of rpoS transcription $(36,37)$. We constructed tet insertion / deletions in cytR, fnr and dps. No role for these three genes in the control of rpoS transcription was indicated, based on equivalent activity of $\mathrm{P}_{r p o s}$-lac [op] in the wild type and mutant backgrounds (data not shown). The roles of Fis and CRP were further investigated.

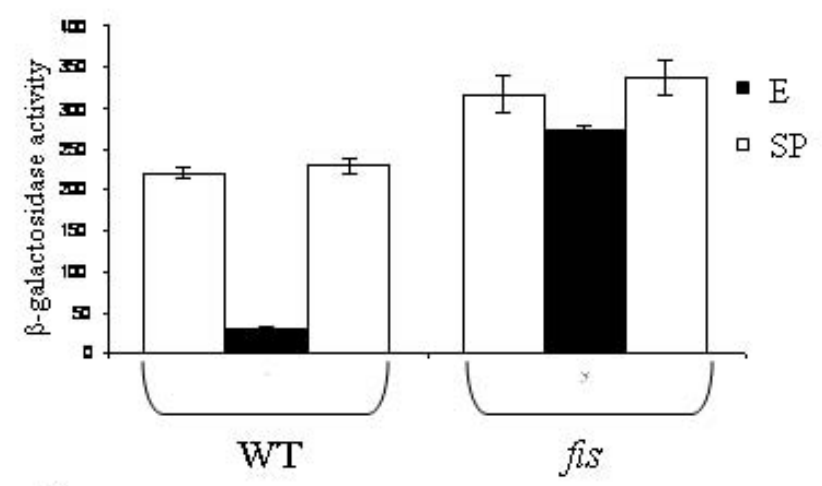

B.

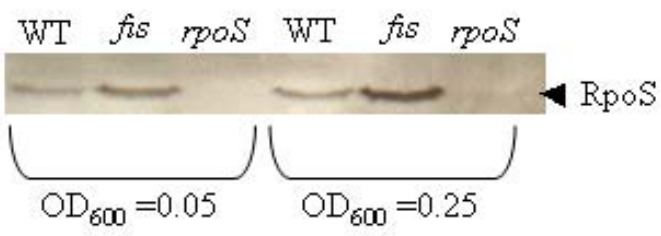

Figure 13. RpoS protein is elevated in a fis null mutant.

(A) Wild type and fis mutant strains carrying $\mathrm{P}_{r p o s}$-lac [op] (TE8744 and TE8764 respectively) were grown to SP and the $\beta$-galactosidase activity determined; the same cultures were diluted into pre-warmed fresh LB medium to allow exponential growth into SP, and the activity determined for both E and SP. (B) Wild type (TE6285) and fis mutant (TE8768) strains were analyzed for RpoS protein at two exponential phase time points as described in Materials and Methods. 
$\mathbf{P}_{\text {rpos }}$ expression and RpoS protein level are elevated in a fis mutant. One of the most convincing (and intriguing) potential binding sites was a strong Fis site centered at bp -50 with respect to the transcriptional start. Repression of $r p o S$ transcription by Fis was an attractive hypothesis because the amount of Fis varies substantially at different points along the growth curve: Fis is abundant during exponential phase when RpoS is at a low level, whereas the Fis level drops sharply in SP as RpoS is induced $(2,6)$. To investigate the role of Fis in rpoS regulation, we tested the effect of a fis insertion / deletion (49) on the activity of $\mathrm{P}_{r p o s}$-lac [op]. During exponential phase, $\mathrm{P}_{r p o s}$-lac expression was 9fold higher in the fis mutant than in wild type (Fig. 13A). This large increase was evident throughout exponential phase, yet there was little difference from wild type during SP. Since the negative effect of Fis is restricted to exponential phase, this finding supports a role for Fis as negative regulator of $r p o S$ at the transcriptional level. The activity of $\mathrm{P}_{\text {rpos }}$-lac [op] in both wild type and fis mutant backgrounds did not change even after extended growth in exponential phase, achieved by three repeated dilutions of dividing cells into pre-warmed fresh LB medium, (data not shown).

Western blot analysis was used to determine the abundance of RpoS protein in the fis mutant. Samples for Western analysis were taken two generations after dilution $\left(\mathrm{OD}_{600}=0.05\right)$ and near midexponential phase, $\mathrm{OD}_{600}=0.2$ (Fig. 13B). At both exponential time points RpoS protein abundance appeared significantly higher in the fis mutant (3-4 fold as measured by densitometry). RpoS protein observed in the fis mutant after 24 hours of growth, a time when Fis levels are at a minimum, was indistinguishable from wild type (data not shown). We also observed increased exponential phase expression of the RpoS-dependent dependent reporters katE-lac [op] (three-fold) and proV-lac [op] (four-fold) in the fis mutant, consistent with the Western blot analysis of RpoS protein. Again, the 
specificity of the fis effect to exponential phase defines a regulatory role.

Complementation of the fis mutation. To confirm that the increase in rpoS transcription in the fis mutant was due to the absence of Fis, exponential phase activity of $\mathrm{P}_{\text {rpos }}$-lac [op] was measured in wild type and fis mutant backgrounds harboring either the single-copy fis expression plasmid, pFis349, or the empty vector control pGS349 $(32,71)$. The nine-fold elevation of $r p o S$ transcription in the fis mutant was completely eliminated by pFis349 (Fig. 14). The presence of pFis349 in the wild type strain slightly decreased $\mathrm{P}_{r p o S}-l a c$ activity, consistent with the idea that Fis represses $r p o S$ transcription. The wild type and fis strains containing the control plasmid exhibited similar activities compared to the plasmid-free strains.

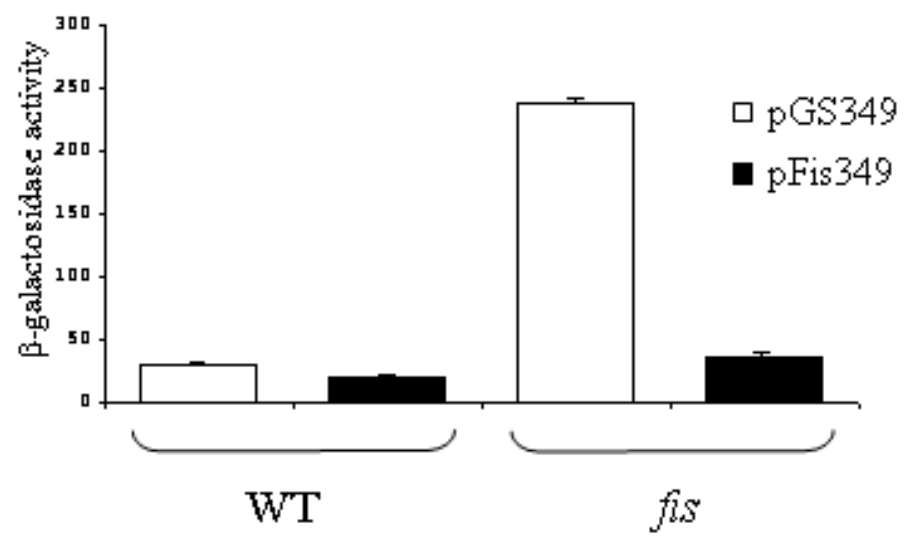

Figure 14. Complementation of the fis mutant.

LT2 wild type and fis mutant strains containing the $\mathrm{P}_{\text {rpos }}$-lac [op] fusion, and also harboring pFis349 ( $^{+}{ }^{+}$; TE8916, TE8917) or its vector control pGS349 (TE8911, TE8912) were grown at $37^{\circ} \mathrm{C}$ in $\mathrm{LB}$ medium containing ampicillin. Activity of $\beta$ galactosidase was determined for cultures grown to $\mathrm{OD}_{600}=0.25$. 


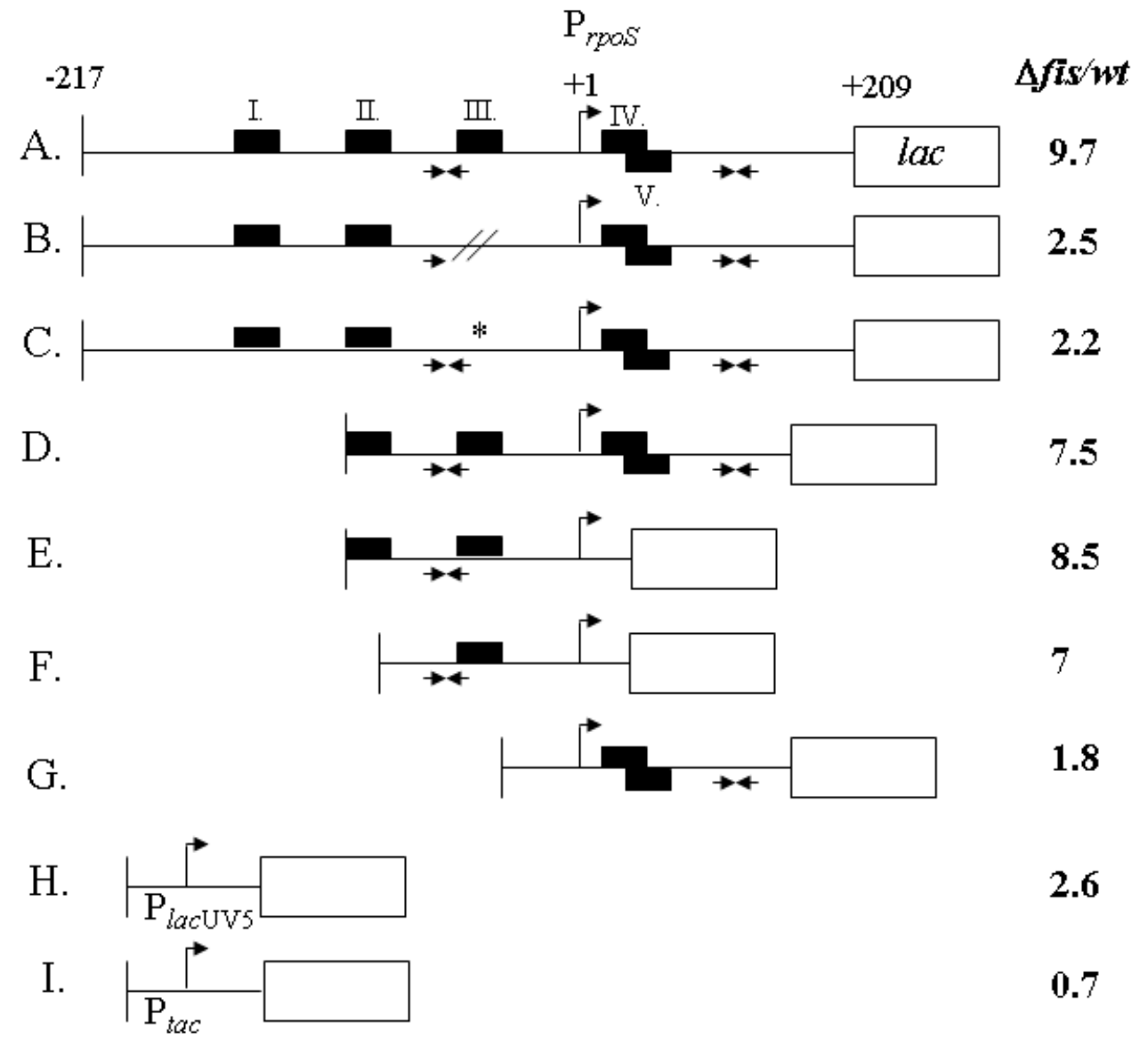

Figure 15. Transcriptional regulation of $r p o S$ by Fis depends on Fis site III.

Construct A represents the full-length, wild type $\mathrm{P}_{\text {rpos }}$-lac [op] fusion. Predicted Fis binding sites (labeled I to V) are represented by black boxes, and predicted CRP half-sites are shown using straight arrows. The bent arrow represents the transcriptional start. Construct $\mathrm{B}$ is a derivative of construct A carrying an in-frame deletion of the highscoring Fis site III. Construct $\mathrm{C}$ is identical to construct $\mathrm{A}$ except for a set of 3 point mutations (represented by an asterisk) altering conserved nucleotides of Fis site III. In constructs $\mathrm{D}, \mathrm{E}, \mathrm{F}$, and $\mathrm{G}$ additional segments of this region are deleted as shown. Constructs $\mathrm{H}$ and I are control transcriptional fusions driven by either the $\mathrm{P}_{\text {lac UV5 }}$ or $\mathrm{P}_{\text {tac }}$ promoter. These constructs were assayed during exponential phase growth in LB medium, in wild type and fis mutant backgrounds. Results are plotted as the ratio of activity in the $\Delta$ fis strain to the activity observed in wild type. Wild type and fis mutant strains are, respectively, as follows: A, (TE8744, TE8764); B, (TE8899, TE8900); C, (TE8887, TE8888); D, (TE8971, TE8972); E, (TE8868, TE8869); F, (TE9083, TE9096); G, (TE8947, TE8949); H, (TE6676, TE8766); I, (TE6675, TE8948). 


\section{Elevation of $\mathbf{P}_{r p o s}$ activity in a fis mutant depends on a predicted Fis binding site. Fis-}

binding DNA sequences have been analyzed by using information analysis (27). In this method, known binding sites are first aligned based on highly conserved nucleotides. The nucleotide distribution at each position within the alignment is then used to derive a weight matrix according to classic information theory $(61,65)$. To determine the quantitative "goodness" of a candidate site, the relevant entries for each position in the weight matrix are summed. If a particular position were completely conserved within the known sites, a correct match in the candidate site would contribute 2 bits to its score. Characterized Fis sites have total scores that range from 2.5 to 15.7, and the well-studied ones in hin proximal to the hixL site have scores of 8-9 (27), while total scores for random sequence average 0 . Information analysis has been shown to accurately predict new Fis DNA binding sites (27).

Software to perform the calculations is available (http://www.lecb.ncifcrf.gov/ toms/delila.html) but we chose to implement these relatively simple computations as a Python script (unpublished data). Our analysis predicted a single high-scoring Fis binding site centered at position -50 with respect to the $\mathrm{P}_{\text {rpos }}$ start site (bit score of 10.9; TCTGCACAAAATTCCACCGTT, Fig. 12; Fis site III in Fig. 15). Only 10 out of 60 characterized Fis sites have a higher score. Weaker Fis sites near $\mathrm{P}_{\text {rpos }}$ were also predicted (scores from 4.1, Fig. 12B). In fact, we found 83 sites with scores equal to or greater than rpoS Fis site III within the first $10^{6}$ bp of the E. coli genomic sequence. Nevertheless, since Fis is an abundant DNA binding protein at its peak levels (up to 100,000 monomers per cell), it is possible that most predicted sites are actually bound by Fis protein during exponential growth. 
The contribution of Fis site III to rpoS regulation was determined by constructing mutant derivatives of the standard $\mathrm{P}_{\text {rpos }}$-lac [op] fusion (Fig. 15, construct A). This construct displays over a nine-fold elevation during exponential growth in the fis mutant, compared to a wild type background. Construct B contains a deletion of Fis site III (in frame for $n l p D, \Delta-60$ to -40 ) in which half of a putative CRP binding site, which overlaps Fis site III, was also deleted (Fig. 12 and 15). This construct was nearly blind to the effect of the fis mutation and demonstrated a ratio of activity (fis mutant / wild

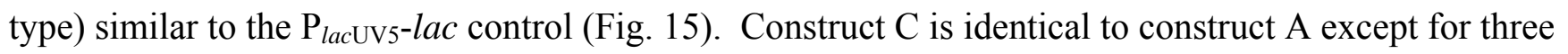
point mutations at critical base pairs in Fis-site III [A(-53)G, T(-48)G, C(-43)G; bit score of -3.3) that do not alter the predicted CRP site. This fusion also failed to show elevated expression of $\mathrm{P}_{\text {rpos }}$ in the fis mutant. Both of the Fis site III mutations (constructs B and C) confer a modest defect in expression in the fis mutant, as compared to construct A (1.5 and 2.4 fold decrease, respectively), perhaps because these mutations also affect basal promoter activity slightly. All fusions that retained the intact Fis site III were subject to control by Fis (Fig. 15 constructs A, D, E and F). Conversely, constructs in which Fis site III was altered or deleted (Fig. 15 constructs B, C and G) were independent of regulation by Fis. We used the $\mathrm{P}_{\text {tac }}$ promoter as a second control in addition to $\mathrm{P}_{\text {lac } \mathrm{UVS}}$; its activity was not increased and in fact was faintly depressed in the fis background.

Fis protein binds to the $\mathbf{P}_{r p o s}$ region. Next, we characterized Fis binding in the $\mathrm{P}_{r p o s}$ promoter region using gel-shift analysis. All binding reactions were performed in the presence of the non-specific competitor DNA, poly [d(I-C)]. As a control for these studies, we first demonstrated binding of purified Fis protein (a gift from R. Johnson) to the E. coli rroP $_{2}$ promoter region over the concentration range reported by Xu and Johnson (72). 
PCR products corresponding to three adjacent regions near $\mathrm{P}_{\text {rpos }}$ (Fig. 16, fragments $\mathrm{A}, \mathrm{B}$, and C) were used as binding targets. Fis bound to each of the fragments, notably with apparent affinities that reflected the score for the predicted Fis site(s) carried on each fragment. Fragment B includes Fis site III required for the in vivo effect of Fis on rpoS (Fig. 15). This fragment exhibited binding at a low concentration of Fis $(32 \mathrm{nM})$ and greater than $90 \%$ of the DNA target was in the bound form at $325 \mathrm{nM}$ Fis. In marked contrast, fragments $\mathrm{A}$ and $\mathrm{C}$ demonstrate $15 \%$ or less than $1 \%$, respectively, of bound target at the same Fis concentration. To further define the contribution of Fis site III, fragment B* was generated from a template carrying the three point mutations in site III that block Fis regulation in vivo. At a higher Fis concentration, in which the wild type B target was essentially all (98\%) in the bound form, the mutant target $\mathrm{B}^{*}$ was predominantly unbound. The $\mathrm{B}^{\Delta}$ target that has a $21 \mathrm{bp}$ deletion of Fis site III also lost the ability to bind Fis. These results suggest that Fis acts directly as a repressor of the $\mathrm{P}_{r p o s}$ promoter.

Testing the interaction of CRP and Fis in transcriptional regulation of rpoS. In E. coli, cAMP-CRP is reported as a negative regulator of $r p o S$ transcription during exponential phase while during entry to SP, the complex may activate transcription $(28,39)$. A motif search of the $\mathrm{P}_{r p o s}$ promoter region by the method of Schneider et al. (61) confirmed two putative CRP binding sites that were also previously predicted in E. coli (37). The higher-scoring of the two sites is centered at -63.5 and actually overlaps Fis site III (Fig. 12). This placement suggests a potential relationship between Fis and CRP in the regulation of rpoS transcription. Coordinate transcriptional regulation between Fis and CRP has been reported for several systems $(14,72)$. 

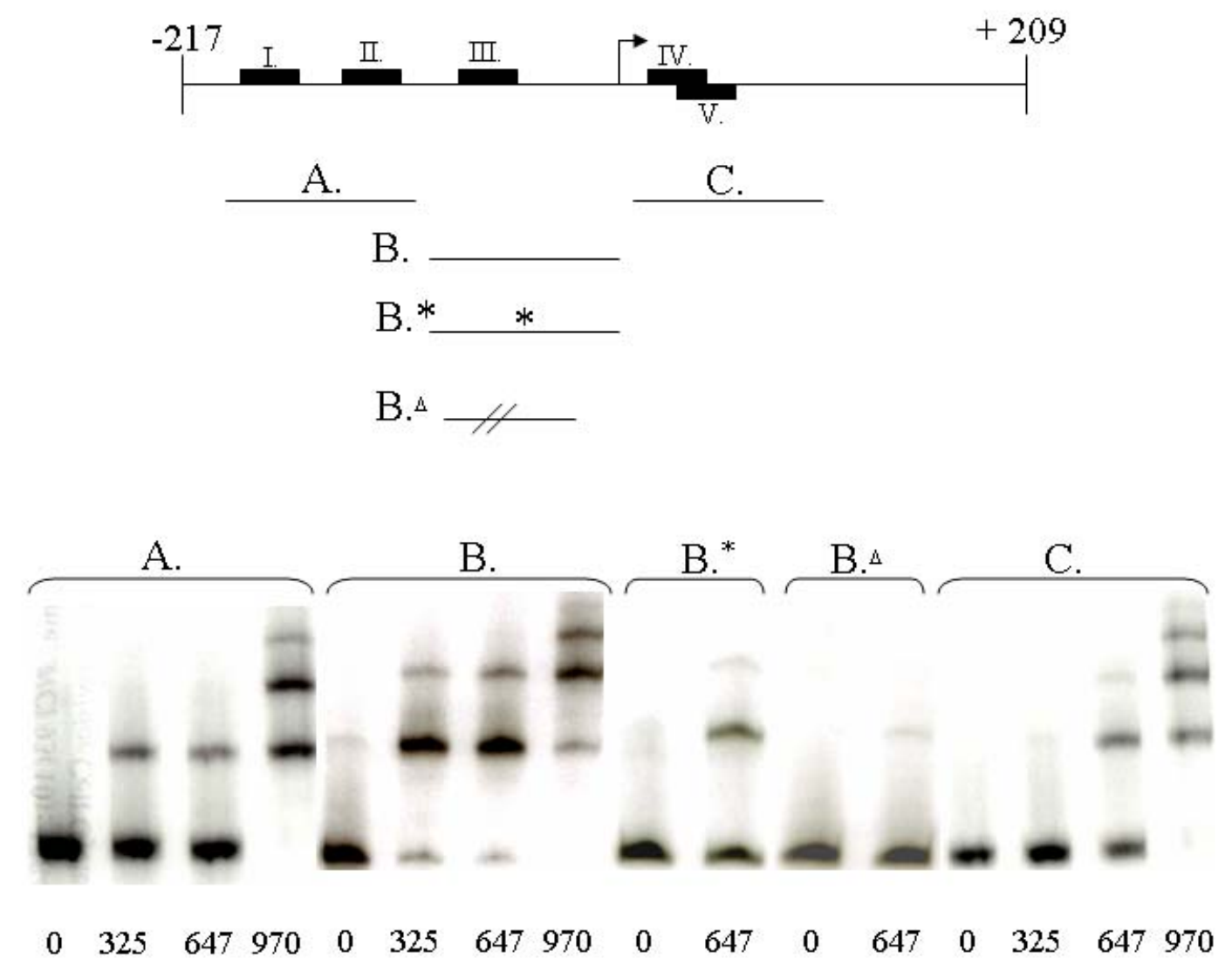

Fis (nM)

Figure 16. Fis binds several DNA sites near $\mathbf{P}_{\text {rpos }}$ in vitro.

Electrophoretic mobility shift assay analysis of Fis protein binding to the $\mathrm{P}_{r p o s}$ region. A map of the region is depicted at the top with black boxes representing predicted Fis binding sites (labeled I-V). PCR was used to generate targets for shift assays which are labeled A through C. For $\mathrm{B}^{*}$ and $\mathrm{B}^{\Delta}$, respectively, the asterisk and the slanted lines represent either a set of 3 point mutations altering Fis site III or a deletion of that site. Radiolabeled fragments were incubated with increasing concentrations of purified Fis protein and analyzed by electrophoresis on a native polyacrylamide gel as described previously (72). 
To investigate the role of CRP, we measured expression of $\mathrm{P}_{\text {rpos }}$-lac [op] during exponential growth in strains bearing the indicated mutations (Fig. 17). These experiments were performed at $37^{\circ} \mathrm{C}$ in buffered LB medium supplemented with $0.2 \%$ glucose to minimize any growth deficiency of the cya and crp mutants. The cya and crp mutants both demonstrated a three-fold increase in rpoS transcription during exponential phase. When a mutant $c r p^{*}$ gene encoding a constitutively active form of CRP was introduced into the cya mutant background, wild type expression was restored. In the fis crp double mutant, only a slight increase in $\mathrm{P}_{\text {rpos }}$-lac [op] expression was observed compared to the fis single mutant. Furthermore, in the fis crp double mutant $\mathrm{P}_{\text {rpos }}$-lac [op] expression was substantially elevated compared to the crp single mutant. These results are consistent with a model in which most Fis regulation of rpoS is independent of CRP function, and is not mediated through, for example, competitive binding of the two regulators at the overlapping Fis III and CRP sites.

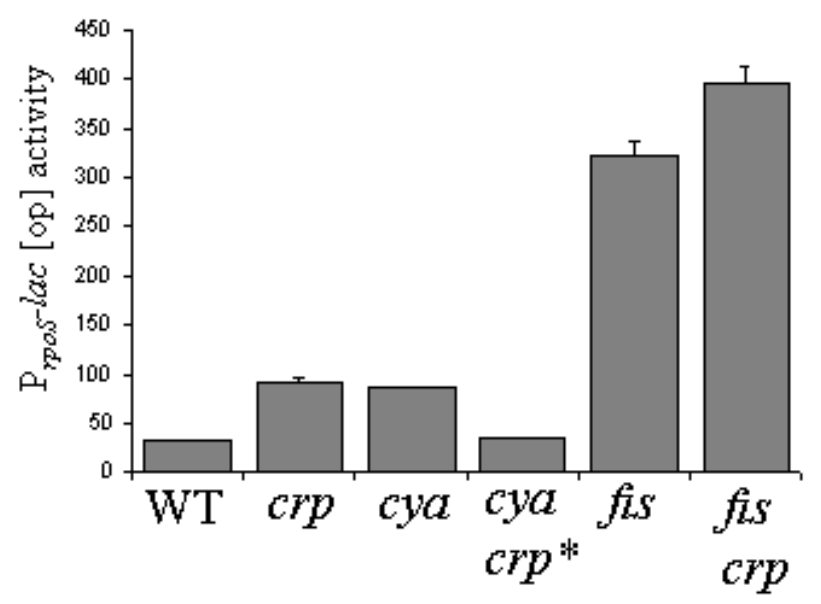

Figure 17. CRP and cAMP influence rpoS transcription.

$\mathrm{P}_{r p o s}-\mathrm{lac}[\mathrm{op}]$ activity was assayed during exponential phase $\left(\mathrm{OD}_{600}=0.25\right)$ in strains containing the indicated mutations. Growth was at $37^{\circ} \mathrm{C}$ in buffered $\mathrm{LB}$ supplemented with $0.2 \%$ glucose. The $c r p *$ allele encodes a constitutively active form of the CRP protein (independent of cAMP). 


\section{Discussion}

The sigma factor RpoS has been described as the "master regulator of the general stress response" (28). It is noteworthy that this transcriptional regulatory protein is itself up-regulated in response to a number of different stresses, by pathways that act on diverse targets including transcription, translation, protein stability and protein activity. The SP induction of RpoS in rich medium is a dramatic effect, whether observed by Western blot of RpoS protein (25) or in a number of studies employing rpoS-lac fusions including our own work, where we estimate the magnitude of the response at 30 -fold $(28,29$, unpublished data). What seems surprising is that various stimuli induce RpoS by such different mechanisms. Starvation for carbon seems to involve mainly stabilization of the protein against attack by the ClpXP protease $(36,66,73)$. Osmotic shock involves both protein stabilization (44) and a post-transcriptional effect dependent mainly on DsrA RNA and the Hfq protein. This pathway does not lead to an increase in the amount of DsrA (38). In contrast, low temperature leads to increased synthesis of DsrA RNA and Hfq-dependent activation of RpoS translation (54). Finally, SP induction of RpoS in rich medium involves both transcriptional and post-transcriptional components $(28,29)$.

We initially eliminated the ClpXP-MviA degradation pathway as a regulator of SP induction of RpoS in rich medium (Fig. 9). This conclusion is consistent with the results of Pratt et al. (52) who demonstrated increased RpoS during both exponential and SP in a sprE mutant of E. coli grown in LB. However, during growth of E. coli in minimal medium, the RpoS protein has a short half-life (1 min) and upon osmotic challenge, starvation or switch to an acidic $\mathrm{pH}$, 
protein stabilization is responsible for RpoS induction $(7,44,73)$. These reports are consistent with only a small transcriptional induction of $\mathrm{P}_{r p o s}$ during growth to SP in minimal medium (Table 7). In rich media (LB and BHI) control of SP induction of RpoS is exerted at the transcriptional and translational level while in minimal medium proteolysis seems to play the major role.

Consistent with previous reports, rpoS is transcribed from two promoter regions $\mathrm{P}_{n l p D}$ and $\mathrm{P}_{r p o S}(35,51) . \mathrm{P}_{n l p D}$ is a minor contributor during both growth and SP and does not exhibit SP induction beyond the $\mathrm{P}_{\text {lac } \mathrm{UV} 5}$ control (Fig. 10, data not shown). $\mathrm{P}_{r p o s}$ is the major rpoS promoter during growth and SP, when increased transcriptional activity coincides with elevated RpoS. Three lines of evidence suggest that $\sigma^{70}$ recognizes $\mathrm{P}_{\text {rpos }}$ in vivo: (i) the presence of an apparent $\sigma^{70}$ promoter sequence (Fig. 12); (ii) RpoS does not contribute to expression from its own promoter (Fig. 11); (iii) RNA polymerase holoenzyme $\left(\sigma^{70}\right)$ transcribes this promoter in vitro (data not shown).

The elevation of $\mathrm{P}_{r p o s}$ activity in the fis mutant during exponential phase eliminated SP transcriptional induction, an effect that can be totally complemented by plasmid-encoded Fis. The standard $\mathrm{P}_{r p o s}$ fusion (Fig. 15, construct A) contains 5 predicted Fis binding sites. Fis binding was demonstrated to at least 3 sites (Fig. 16) suggesting a nucleoprotein complex forms near $\mathrm{P}_{r p o s}$ in vivo. However only Fis site III, positioned at -50, was required for the regulatory effect. The importance of a single Fis binding site near transcriptional start sites has been reported for two other promoters, $\mathrm{P}_{\text {fis }}$ and $\mathrm{P} 2_{\text {prop. }}(50,72)$. In each case Fis binds to several positions although nearly all regulation is conferred by a single Fis binding site centered at -42 
$\left(\mathrm{P}_{f i s}\right)$ and $-41\left(\mathrm{P} 2_{\text {prop }}\right)$ from the transcriptional start sites $(41,50,72)$. Transcriptional regulation by Fis at $\mathrm{P}_{\text {fis }}$ causes repression, possibly by blocking RNA polymerase interactions at the -35 region. During growth, the fis mutant displayed a nine-fold elevation of $\mathrm{P}_{r p o s}$ activity although the increase of RpoS protein was estimated at three to four-fold (Fig. 13). This suggests that additional (post-transcriptional) regulation may prevent some of the expected increase in protein levels, thereby making both rpoS transcription and translation rate limiting.

Concerning the role of CRP-cAMP in the regulation of $\mathrm{P}_{r p o s}$, we suggest that it functions as a repressor although its effect is modest (two to three-fold). The relationship between CRP and Fis appears to be one of Fis epistasis. The fis crp double mutant does not display an additive effect on $\mathrm{P}_{r p o s}$ activity but there is only a slight increase over the large effect of the fis single mutant. From this it seems that Fis regulation does not require CRP and that full CRP regulation is hindered in the absence of Fis. Further experimentation is necessary to define the role of CRP in the regulation of rpoS transcription.

In this study, we document a role for the DNA-binding protein, Fis, as a negative regulatory element for RpoS, acting at the transcriptional level. This model is intuitive, because Fis abundance varies inversely with RpoS. Synthesis of Fis is under transcriptional control and Fis abundance varies dramatically from undetectable in SP to over 40,000 dimers per cell upon dilution into fresh medium $(2,6,50)$. In E. coli, Fis displays auto-regulation in which Fis protein competes with RNA polymerase for binding to the fis promoter thus repressing its own transcription (6). This auto-regulatory effect is less pronounced in S. enterica (50). In both organisms growth-phase expression of fis is thought to occur by a mechanism apparently 
involving a non-optimal -35 to -10 region and specific base pairs near the transcriptional start site (69). It has been demonstrated that normal regulation is also dependent upon CRP and in vitro results suggest fis promoter activation in the absence of Fis, while Fis and CRP act synergistically as transcriptional repressors (47).

We believe that Fis probably acts directly as a repressor given that a specific site positioned at -50 is necessary for complete repression and that this site is specifically bound in vitro. Fis activates transcription of rRNA and many other genes including some involved in replication, so it is conceivable that Fis also works indirectly. Further investigations into the regulation of Fis expression would provide a greater understanding of the interplay of global regulators in physiological adaptation.

Acknowledgements. This work was supported by Public Health Service grant GM63616. We sincerely thank Reid Johnson for the gift of purified Fis protein and the individuals cited in the text for bacterial strains. We also thank Joan Olson for comments and suggestions in preparation of this manuscript.

\section{Literature Cited}

1. Ailion, M., T. A. Bobik, and J. R. Roth. 1993. Two global regulatory systems (Crp and Arc) control the cobalamin/propanediol regulon of Salmonella typhimurium. J. Bacteriol. 175:7200-8.

2. Ali Azam, T., A. Iwata, A. Nishimura, S. Ueda, and A. Ishihama. 1999. Growth phase-dependent variation in protein composition of the Escherichia coli nucleoid. J. Bacteriol. 181:6361-70. 
3. Auner, H., M. Buckle, A. Deufel, T. Kutateladze, L. Lazarus, R. Mavathur, G. Muskhelishvili, I. Pemberton, R. Schneider, and A. Travers. 2003. Mechanism of transcriptional activation by FIS: role of core promoter structure and DNA topology. J. Mol. Biol. 331:331-44.

4. Ball, C. A., and R. C. Johnson. 1991. Efficient excision of phage lambda from the Escherichia coli chromosome requires the Fis protein. J. Bacteriol. 173:4027-31.

5. Ball, C. A., and R. C. Johnson. 1991. Multiple effects of Fis on integration and the control of lysogeny in phage lambda. J. Bacteriol. 173:4032-8.

6. Ball, C. A., R. Osuna, K. C. Ferguson, and R. C. Johnson. 1992. Dramatic changes in Fis levels upon nutrient upshift in Escherichia coli. J. Bacteriol. 174:8043-56.

7. Bearson, S. M., W. H. Benjamin, Jr., W. E. Swords, and J. W. Foster. 1996. Acid shock induction of RpoS is mediated by the mouse virulence gene mviA of Salmonella typhimurium. J. Bacteriol. 178:2572-9.

8. Becker, G., E. Klauck, and R. Hengge-Aronis. 2000. The response regulator RssB, a recognition factor for sigmaS proteolysis in Escherichia coli, can act like an anti-sigmaS factor. Mol. Microbiol. 35:657-66.

9. Benjamin, W. H., Jr., X. Wu, and W. E. Swords. 1996. The predicted amino acid sequence of the Salmonella typhimurium virulence gene mviAA(+) strongly indicates that MviA is a regulator protein of a previously unknown S. typhimurium response regulator family. Infect. Immun. 64:2365-7.

10. Berkowitz, D. J., Hushon, J. M., Whitfield, H. J., Roth J., Ames, B. N. 1968. Procedure for identifying nonsense mutants. J. Bacteriol. 96:215-220.

11. Bochner, B. R., Ames, B. N. 1982. Complete analysis of cellular necleotides by twodimensional thin layer chromatography. J. Biol. Chem. 257:9759-9769.

12. Bordes, P., J. Bouvier, A. Conter, A. Kolb, and C. Gutierrez. 2002. Transient repressor effect of Fis on the growth phase-regulated osmE promoter of Escherichia coli K12. Mol. Genet. Genomics 268:206-13.

13. Brown, L., and T. Elliott. 1996. Efficient translation of the RpoS sigma factor in Salmonella typhimurium requires host factor I, an RNA-binding protein encoded by the hfq gene. J. Bacteriol. 178:3763-70. 
14. Browning, D. F., C. M. Beatty, E. A. Sanstad, K. E. Gunn, S. J. Busby, and A. J. Wolfe. 2004. Modulation of CRP-dependent transcription at the Escherichia coli acsP2 promoter by nucleoprotein complexes: anti-activation by the nucleoid proteins FIS and IHF. Mol. Microbiol. 51:241-54.

15. Cunning, C., L. Brown, and T. Elliott. 1998. Promoter substitution and deletion analysis of upstream region required for $r p o S$ translational regulation. J. Bacteriol. 180:4564-70.

16. Cunning, C., and T. Elliott. 1999. RpoS synthesis is growth rate regulated in Salmonella typhimurium, but its turnover is not dependent on acetyl phosphate synthesis or PTS function. J. Bacteriol. 181:4853-62.

17. Datsenko, K. A., and B. L. Wanner. 2000. One-step inactivation of chromosomal genes in Escherichia coli K-12 using PCR products. Proc. Natl. Acad. Sci. U S A 97:6640-5.

18. Davis, R. W., Botstein, D., Roth, J. 1980. Advanced Bacterial Genetics. Cold Spring Harbor, Cold Spring Harbor, N. Y.

19. Elliott, T. 1992. A method for constructing single-copy lac fusions in Salmonella typhimurium and its application to the hemA-prfA operon. J. Bacteriol 174:245-53.

20. Esposito, D., and G. F. Gerard. 2003. The Escherichia coli Fis protein stimulates bacteriophage lambda integrative recombination in vitro. J. Bacteriol. 185:3076-80.

21. Falconi, M., N. P. Higgins, R. Spurio, C. L. Pon, and C. O. Gualerzi. 1993. Expression of the gene encoding the major bacterial nucleotide protein $\mathrm{H}-\mathrm{NS}$ is subject to transcriptional auto-repression. Mol. Microbiol. 10:273-82.

22. Fang, F. C., S. J. Libby, N. A. Buchmeier, P. C. Loewen, J. Switala, J. Harwood, and D. G. Guiney. 1992. The alternative sigma factor katF (rpoS) regulates Salmonella virulence. Proc. Natl. Acad. Sci. U S A 89:11978-82.

23. Farewell, A., K. Kvint, and T. Nystrom. 1998. Negative regulation by RpoS: a case of sigma factor competition. Mol. Microbiol. 29:1039-51.

24. Filutowicz, M., W. Ross, J. Wild, and R. L. Gourse. 1992. Involvement of Fis protein in replication of the Escherichia coli chromosome. J. Bacteriol. 174:398-407. 
25. Gentry, D. R., V. J. Hernandez, L. H. Nguyen, D. B. Jensen, and M. Cashel. 1993. Synthesis of the stationary-phase sigma factor sigma s is positively regulated by ppGpp. J. Bacteriol. 175:7982-9.

26. Gille, H., J. B. Egan, A. Roth, and W. Messer. 1991. The FIS protein binds and bends the origin of chromosomal DNA replication, oriC, of Escherichia coli. Nucleic Acids Res. 19:4167-72.

27. Hengen, P. N., S. L. Bartram, L. E. Stewart, and T. D. Schneider. 1997. Information analysis of Fis binding sites. Nucleic Acids Res. 25:4994-5002.

28. Hengge-Aronis, R. 2002. Signal transduction and regulatory mechanisms involved in control of the sigma(S) (RpoS) subunit of RNA polymerase. Microbiol. Mol. Biol. Rev. 66:373-95, table of contents.

29. Hirsch, M., and T. Elliott. 2002. Role of ppGpp in rpoS stationary-phase regulation in Escherichia coli. J. Bacteriol. 184:5077-87.

30. Hughes, K. T., and J. R. Roth. 1984. Conditionally transposition-defective derivative of Mu d1(Amp Lac). J. Bacteriol. 159:130-7.

31. Johnson, R. C., Bruist, M. F., Simon, R. I. 1986. Host protein requirements for in vitro site specific DNA inversion. Cell 46:531-539.

32. Jourdan, A. D., and G. V. Stauffer. 1999. Genetic analysis of the GevA binding site in the gcvA control region. Microbiology 145 ( Pt 8):2153-62.

33. Keane, O. M., and C. J. Dorman. 2003. The gyr genes of Salmonella enterica serovar Typhimurium are repressed by the factor for inversion stimulation, Fis. Mol. Genet. Genomics 270:56-65.

34. Koch, C., and R. Kahmann. 1986. Purification and properties of the Escherichia coli host factor required for inversion of the $\mathrm{G}$ segment in bacteriophage $\mathrm{Mu}$. J. Biol. Chem. 261:15673-8.

35. Lange, R., D. Fischer, and R. Hengge-Aronis. 1995. Identification of transcriptional start sites and the role of ppGpp in the expression of $r p o S$, the structural gene for the sigma S subunit of RNA polymerase in Escherichia coli. J. Bacteriol. 177:4676-80. 
36. Lange, R., and R. Hengge-Aronis. 1994. The cellular concentration of the sigma S subunit of RNA polymerase in Escherichia coli is controlled at the levels of transcription, translation, and protein stability. Genes Dev. 8:1600-12.

37. Lange, R., and R. Hengge-Aronis. 1991. Identification of a central regulator of stationary-phase gene expression in Escherichia coli. Mol. Microbiol. 5:49-59.

38. Majdalani, N., C. Cunning, D. Sledjeski, T. Elliott, and S. Gottesman. 1998. DsrA RNA regulates translation of RpoS message by an anti-antisense mechanism, independent of its action as an antisilencer of transcription. Proc. Natl. Acad. Sci. U S A 95:12462-7.

39. McCann, M. P., J. P. Kidwell, and A. Matin. 1991. The putative sigma factor KatF has a central role in development of starvation-mediated general resistance in Escherichia coli. J. Bacteriol. 173:4188-94.

40. McClelland, M., K. E. Sanderson, J. Spieth, S. W. Clifton, P. Latreille, L. Courtney, S. Porwollik, J. Ali, M. Dante, F. Du, S. Hou, D. Layman, S. Leonard, C. Nguyen, K. Scott, A. Holmes, N. Grewal, E. Mulvaney, E. Ryan, H. Sun, L. Florea, W. Miller, T. Stoneking, M. Nhan, R. Waterston, and R. K. Wilson. 2001. Complete genome sequence of Salmonella enterica serovar Typhimurium LT2. Nature 413:852-6.

41. McLeod, S. M., S. E. Aiyar, R. L. Gourse, and R. C. Johnson. 2002. The C-terminal domains of the RNA polymerase alpha subunits: contact site with Fis and localization during co-activation with CRP at the Escherichia coli proP P2 promoter. J. Mol. Biol. 316:517-29.

42. Metcalf, W. W., W. Jiang, L. L. Daniels, S. K. Kim, A. Haldimann, and B. L. Wanner. 1996. Conditionally replicative and conjugative plasmids carrying lacZ alpha for cloning, mutagenesis, and allele replacement in bacteria. Plasmid 35:1-13.

43. Miller, J. H. 1972. Experiments in molecular genetics. Cold Spring Harbor laboratory, Cold Spring Harbor, N.Y.

44. Muffler, A., D. Fischer, S. Altuvia, G. Storz, and R. Hengge-Aronis. 1996. The response regulator RssB controls stability of the sigma(S) subunit of RNA polymerase in Escherichia coli. Embo J. 15:1333-9. 
45. Mulvey, M. R., and P. C. Loewen. 1989. Nucleotide sequence of katF of Escherichia coli suggests KatF protein is a novel sigma transcription factor. Nucleic Acids Res. 17:9979-91.

46. Mulvey, M. R., J. Switala, A. Borys, and P. C. Loewen. 1990. Regulation of transcription of katE and katF in Escherichia coli. J. Bacteriol. 172:6713-20.

47. Nasser, W., R. Schneider, A. Travers, and G. Muskhelishvili. 2001. CRP modulates fis transcription by alternate formation of activating and repressing nucleoprotein complexes. J. Biol. Chem. 276:17878-86.

48. Neidhardt, F. C., Bloch, P. L., Smith, D. F. 1974. Culture medium for enterobacteria. J. Bacteriol. 119:736-747.

49. Numrych, T. E., R. I. Gumport, and J. F. Gardner. 1991. A genetic analysis of Xis and FIS interactions with their binding sites in bacteriophage lambda. J. Bacteriol. 173:5954-63.

50. Osuna, R., D. Lienau, K. T. Hughes, and R. C. Johnson. 1995. Sequence, regulation, and functions of fis in Salmonella typhimurium. J. Bacteriol. 177:2021-32.

51. Paesold, G., and M. Krause. 1999. Analysis of rpoS mRNA in Salmonella dublin: identification of multiple transcripts with growth-phase-dependent variation in transcript stability. J. Bacteriol. 181:1264-8.

52. Pratt, L. A., and T. J. Silhavy. 1996. The response regulator SprE controls the stability of RpoS. Proc. Natl. Acad. Sci. U S A 93:2488-92.

53. Pruteanu, M., and R. Hengge-Aronis. 2002. The cellular level of the recognition factor RssB is rate-limiting for sigmaS proteolysis: implications for RssB regulation and signal transduction in sigmaS turnover in Escherichia coli. Mol. Microbiol. 45:1701-13.

54. Repoila, F., and S. Gottesman. 2001. Signal transduction cascade for regulation of RpoS: temperature regulation of DsrA. J. Bacteriol. 183:4012-23.

55. Robison, K., A. M. McGuire, and G. M. Church. 1998. A comprehensive library of DNA-binding site matrices for 55 proteins applied to the complete Escherichia coli K-12 genome. J. Mol. Biol 284:241-54.

56. Ross, W., J. Salomon, W. M. Holmes, and R. L. Gourse. 1999. Activation of Escherichia coli leuV transcription by FIS. J. Bacteriol. 181:3864-8. 
57. Ruiz, N., C. N. Peterson, and T. J. Silhavy. 2001. RpoS-dependent transcriptional control of sprE: regulatory feedback loop. J. Bacteriol. 183:5974-81.

58. Ryan, V. T., J. E. Grimwade, J. E. Camara, E. Crooke, and A. C. Leonard. 2004. Escherichia coli prereplication complex assembly is regulated by dynamic interplay among Fis, IHF and DnaA. Mol. Microbiol. 51:1347-59.

59. Sak, B. D., A. Eisenstark, and D. Touati. 1989. Exonuclease III and the catalase hydroperoxidase II in Escherichia coli are both regulated by the katF gene product. Proc. Natl. Acad. Sci. U S A 86:3271-5.

60. Schellhorn, H. E., and V. L. Stones. 1992. Regulation of katF and katE in Escherichia coli K-12 by weak acids. J. Bacteriol. 174:4769-76.

61. Schneider, T. D., G. D. Stormo, L. Gold, and A. Ehrenfeucht. 1986. Information content of binding sites on nucleotide sequences. J. Mol. Biol. 188:415-31.

62. Schweder, T., K. H. Lee, O. Lomovskaya, and A. Matin. 1996. Regulation of Escherichia coli starvation sigma factor (sigma s) by ClpXP protease. J. Bacteriol. 178:470-6.

63. Silhavy, T. J., Berman M. L., Enquist L. W. 1984. Experiments with gene fusions. Cold Spring Harbor Laboratory, Cold Spring Harbor, N.Y.

64. Simons, R. W., F. Houman, and N. Kleckner. 1987. Improved single and multicopy lac-based cloning vectors for protein and operon fusions. Gene 53:85-96.

65. Stormo, G. D., and G. W. Hartzell, 3rd. 1989. Identifying protein-binding sites from unaligned DNA fragments. Proc. Natl. Acad. Sci. U S A 86:1183-7.

66. Takayanagi, Y., K. Tanaka, and H. Takahashi. 1994. Structure of the 5' upstream region and the regulation of the rpoS gene of Escherichia coli. Mol. Gen. Genet 243:52531.

67. Thompson, J. F., U. K. Snyder, and A. Landy. 1988. Helical-repeat dependence of integrative recombination of bacteriophage lambda: role of the $\mathrm{P} 1$ and $\mathrm{H} 1$ protein binding sites. Proc. Natl. Acad. Sci. U S A 85:6323-7.

68. Wackwitz, B., J. Bongaerts, S. D. Goodman, and G. Unden. 1999. Growth phasedependent regulation of nuoA- $N$ expression in Escherichia coli K-12 by the Fis protein: upstream binding sites and bioenergetic significance. Mol. Gen. Genet. 262:876-83. 
69. Walker, K. A., and R. Osuna. 2002. Factors affecting start site selection at the Escherichia coli fis promoter. J. Bacteriol. 184:4783-91.

70. Weinreich, M. D., and W. S. Reznikoff. 1992. Fis plays a role in Tn5 and IS50 transposition. J. Bacteriol. 174:4530-7.

71. Wilson, R. L., S. J. Libby, A. M. Freet, J. D. Boddicker, T. F. Fahlen, and B. D. Jones. 2001. Fis, a DNA nucleoid-associated protein, is involved in Salmonella typhimurium SPI-1 invasion gene expression. Mol. Microbiol. 39:79-88.

72. Xu, J., and R. C. Johnson. 1995. Fis activates the RpoS-dependent stationary-phase expression of proP in Escherichia coli. J. Bacteriol. 177:5222-31.

73. Zgurskaya, H. I., M. Keyhan, and A. Matin. 1997. The sigma S level in starving Escherichia coli cells increases solely as a result of its increased stability, despite decreased synthesis. Mol. Microbiol. 24:643-51.

74. Zhi, H., X. Wang, J. E. Cabrera, R. C. Johnson, and D. J. Jin. 2003. Fis stabilizes the interaction between RNA polymerase and the ribosomal promoter $\operatorname{rrnB} \mathrm{P} 1$, leading to transcriptional activation. J. Biol. Chem. 278:47340-9.

75. Zhou, Y., and S. Gottesman. 1998. Regulation of proteolysis of the stationary-phase sigma factor RpoS. J. Bacteriol. 180:1154-8. 


\title{
Stationary Phase Regulation of RpoS synthesis in Escherichia coli
}

\author{
Matthew Hirsch and Thomas Elliott* \\ Department of Microbiology, Immunology and Cell Biology \\ West Virginia University Health Science Center \\ Morgantown, WV 26506
}

* Corresponding author

Phone: 304 293-2676

Fax: 304-293-7823

telliott@hsc.wvu.edu 


\section{Abstract}

In enteric bacteria, adaptation to a number of different stresses is mediated by the RpoS protein, one of several sigma-factors that collectively allow a tailored transcriptional response to environmental cues. Stress stimuli including low temperature, osmotic shock, nutrient limitation, and growth to stationary phase (SP), all result in a substantial increase in RpoS abundance and activity. The mechanism of regulation depends on the specific signal, but may occur at the level of transcription, translation, protein activity or targeted proteolysis. In both Escherichia coli and Salmonella enterica, SP induction of RpoS in rich medium is $>30$-fold and includes effects on both transcription and translation but not protein turnover. Recently, we showed that SP control of rpoS transcription in S. enterica involves repression of the major $r p o S$ promoter by the global transcription factor Fis during exponentialphase. Working primarily in E. coli, we now show that 24 nucleotides of the rpoS ribosome-binding sequence (RBS) are necessary and sufficient for the nearly 10 -fold increase in rpoS translation as cells grow to SP. Genetic evidence supports a model in which the paired structure of the RBS enforces regulation. This regulation is conserved between E. coli and S. enterica. When combined with a fis mutation, substitution of the rpoS RBS sequence by the lacZ RBS eliminates nearly all SP induction of RpoS. 


\section{Introduction}

Bacteria maintain intricate signaling networks that sense the environment and adjust cellular physiology accordingly. In Salmonella enterica and Escherichia coli, unfavorable growth conditions (including nutrient limitation, outright starvation, low temperature, osmotic shock, and other stresses) initiate a generalized stress response by triggering increased abundance of the RNA polymerase sigma factor $\operatorname{RpoS}\left(\sigma^{\mathrm{S}}\right.$; reviewed in reference 16). In association with RNA polymerase, RpoS directs transcription of as much as $10 \%$ of the E. coli genome, including genes necessary for stress resistance and virulence $(13,48)$. RpoS thereby serves as the central regulator of the general protective response (16).

The transition to SP is accompanied by morphological and physiological changes resulting in a non-dividing and multiple-stress resistant state. Growth into stationary phase (SP) in rich media, such as Luria-Bertani (LB), leads to a dramatic increase of $>30$-fold in RpoS abundance $(14,17,18,28,33,39)$. In recent work, we characterized transcriptional regulation of rpoS in S. enterica as cells enter SP (18). The mechanism involves Fis, a DNA-binding protein which acts globally as a transcription factor. Fis is itself growth-phase regulated in an inverse relationship to RpoS: the Fis protein is undetectable in SP but rapidly increases to a level of more than 40,000 dimers per cell upon dilution into fresh medium $(1,3,35)$. A strong Fisbinding site near the major $r p o S$ promoter $\left(\mathrm{P}_{r p o S}\right)$, is required for this regulation. Fis likely binds to this site specifically during exponential growth, resulting in repression of $r p o S$ transcription (18). As cells enter SP, Fis disappears, and rpoS transcription increases nearly 10 -fold $(1,18)$. 
The rpoS transcript contains a 565 nucleotide 5' untranslated region (the rpoS leader; 16, 43). This sequence includes an antisense element [leader nucleotides (nt) 461 to 478 ] that can pair with the rpoS RBS to inhibit translation, presumably by blocking ribosome access (8). The antisense element is the reported target of three regulatory RNAs which are thought to alter conformation of the RBS to an “open" position, increasing translation $(22,23,25,31,38$, reviewed in ref. 15). The best-characterized example of regulation of $r p o S$ translation occurs at low temperature and relies on the direct pairing of the antisense element with the 85-nucleotide regulatory RNA, DsrA (42). This interaction activates rpoS translation five to 10 -fold and is mediated by the RNA-binding protein $\operatorname{Hfq}(25,38)$.

In the present study, we show that 24 nucleotides of the rpoS RBS are necessary and sufficient for a nearly 10 -fold increase in $r p o S$ translation as cells grow to SP. Genetic evidence supports a model in which the secondary structure of the RBS is required for regulation. Substitution of this sequence with the RBS of lacZ, in a fis mutant background, virtually eliminates SP induction of RpoS.

\section{Materials and Methods}

Bacterial strains and construction. Most strains used in this study are derived from the wild type E. coli K-12 strain MG1655 (Table 8). The parental strain was CF7968, which is MG1655 that has been corrected to $\mathrm{rph}^{+}$(20) and deleted for lacIZ, obtained from M. Cashel. Phage P1 vir was used for transduction in E. coli by standard methods (41). The katE-lac [op] (operon) fusion used in this work 
has been described previously and is used as a reporter of RpoS activity $(7,9,18)$. All fusions in E. coli are located in single-copy in the trp region of the bacterial chromosome as described previously (12).

We also investigated the behavior of particular rpoS-lacZ constructs in Salmonella enterica serovar Typhimurium. The parental strain was LT2, obtained from J. Roth, or LT2A $(10,18)$. To this end, constructs in E. coli were transduced into a galE mutant of S. enterica by using P1 vir as described previously (12). The phage P22 mutant HT105 / 1 int-201 was then used for transduction in S. enterica by standard methods (11). All fusions in S. enterica are located in single-copy at the putPA locus (12).

Media and growth conditions. Bacteria were grown at $37^{\circ} \mathrm{C}$ in $\mathrm{LB}$ medium (41) and on nutrient agar (NB) plates containing $5 \mathrm{~g}$ of $\mathrm{NaCl}$ per liter, except where indicated. Minimal agar was prepared with NCE medium containing $0.2 \%$ glucose (5). Liquid minimal medium was morpholinepropanesulfonic acid (MOPS) medium (34) as modified (6), supplemented with $0.2 \%$ glucose as the carbon and energy source. Antibiotics were added to final concentrations in selective media as follows: $20 \mu \mathrm{g}$ of chloramphenicol $/ \mathrm{ml}, 50 \mu \mathrm{g}$ of kanamycin sulfate $/ \mathrm{ml}$, and $20 \mu \mathrm{g}$ of tetracycline hydrochloride/ml. X-Gal (5-bromo-4-chloro-3-indolyl- $\beta$-D-galactopyranoside) was used at $50 \mu \mathrm{g} / \mathrm{ml}$.

Fusion construction. We took advantage of our previously described method for making lac transcriptional and translational constructs in which a region of interest is inserted between the promoter $\mathrm{P}_{\text {lacUV5 }}$ and the lacZYA genes (17). This method relies on the high efficiency $\lambda$ Red recombination system of Yu et al (49). A chromosomal template that has the tetAR cassette immediately upstream of 
$\mathrm{P}_{\text {lacUV5 }}$ was amplified by PCR, using the following primer design (all primer elements are listed in order from 5' to 3'): (i) a common upstream 60-mer that contains $40 \mathrm{nt}$ of kan homology to mediate upstream recombination followed by 20 nucleotides of tetA priming sequence; (ii) construct-specific downstream 80-mers contained 40 nucleotides of either lac or rpoS homology for downstream recombination followed by a variable region of interest preceding priming homology within the lacUV5 promoter. The resulting PCR products have the following structure: kan-tetAR-P $\mathrm{P}_{\text {lacUV5-region of }}$ interest-rpoS codons 1-8 or, alternatively, lacZ coding sequence. Products with downstream homology to $r p o S$ were used to transform TE8402 or TE9277 to generate translational or transcriptional fusions respectively. When recombination directly to the lacZ coding sequence was desired, strain TE9059 served as the recipient. Transformants were selected on NB plates containing tetracycline. The region extending from upstream of $\mathrm{P}_{\text {lac UV5 }}$ through the downstream recombination site (lacZ codon 22) was sequenced. All fusions were then backcrossed into E. coli MG1655 $\Delta$ lacIZ. The sequences for primers used in this study are available upon request.

\section{Unselected chromosomal mutations.}

To replace the rpoS RBS with that of lacZ in the rpoS native context (i.e. at the rpoS locus), a strain with $\lambda$ Red was utilized that carries the katE-lac [op] fusion. First, $24 \mathrm{nt}$ surrounding the rpoS RBS (5'-3'), GGGATCACGGGTAGGAGCCACCTT, were substituted with tetAR. Transformants had a Lac- phenotype on NB plates containing tetracycline and Xgal. Next, a 188 bp PCR product was generated from a wild type template using an upstream rpoS primer (position 410 of the rpoS transcript) and a downstream primer that contained (i) 40 nt of downstream homology to allow recombination downstream of the ATG initiation codon of 
rpos; (ii) $17 \mathrm{nt}$ of the lacZ RBS (AATTTCACACAGGAAACAGCT), and (iii) 19 nt of priming homology to the rpoS leader (nt 541-522). This PCR product was used to transform the TetR strain constructed in the first step followed by dilution into fresh medium for overnight growth. Cultures were then diluted and plated on NB containing kanamycin and X-gal. The phenotype of desired transformants was Lac+; these were recovered at a frequency of $>10-4$, and the insertion was confirmed by DNA sequencing. This unmarked substitution was then backcrossed into a MG1655 background by using a recipient strain which contains the katE-lac [op] fusion, $\Delta r p o S:: c a t(\mathrm{CamR})$, and also $\triangle$ cysC::tetAR. The cysC locus is $\approx 6 \mathrm{~kb}$ from $r p o S$ and tightly linked to it by $\mathrm{P} 1$ transduction. Cys $+\mathrm{Lac}+$ transductants were selected on minimal medium plates containing X-gal and the lacZ RBS substitution in the rpoS leader was confirmed again by sequencing.

Typically, our lac [op] constructs contain a RNase III processing site $(7,24)$, which insulates $l a c Z$ expression from variations due to differences in upstream sequences. For the experiments described here, this property is not desirable. A similar non-selective transformation method to that described above was employed to eliminate the processing site from a strain containing the kan- $\mathrm{P}_{\text {lacUV5- }}$ rpoS (codon 8) transcriptional fusion (TE8403). Briefly, the cat gene was used to make an insertiondeletion with loss of $76 \mathrm{bp}$ including the RNase III cleavage site, located in the $170 \mathrm{nt}$ spacer region between rpoS codon 8 and the lacZ RBS. The desired transformants were Lac ${ }^{-}$(TE9274). Next, a PCR product was generated with the following structure: $r p o S$ codons 6-8 followed by 31 nucleotides of the spacer region, the lacZ RBS and 144 nt of the lacZ coding sequence. Transformation of TE9274 with this PCR product followed by screening for $\mathrm{Lac}^{+}$, resulted in a strain that is deleted for the processing 
site and sensitive to chloramphenicol. The relevant region was confirmed by DNA sequencing (TE9277).

The fis gene of E. coli was deleted and substituted with cat using standard methods (18). Regions surrounding the sites of recombination, fis codon 22 and immediately following the fis termination codon, were confirmed by DNA sequencing.

Assay of $\beta$-galactosidase. Cells were centrifuged and resuspended in Z-buffer $(100 \mathrm{mM} \mathrm{NaPO} 4[\mathrm{pH}$ 7.0], $10 \mathrm{mM} \mathrm{KCl}, 1 \mathrm{mM} \mathrm{MgSO}_{4}$ ) and then permeabilized by treatment with sodium dodecyl sulfate (SDS) and chloroform (29). The samples from exponential-phase were concentrated before assay to be approximately equal in density to samples obtained from later times. For all experiments, exponentialphase is defined as $\mathrm{OD}_{600}=0.25$ and $\mathrm{SP}$ is 24 hours after inoculation. Assays were performed in Zbuffer containing $50 \mathrm{mM} \beta$-mercaptoethanol by a kinetic method using a plate reader (Molecular Dynamics). In all experiments, $\beta$-galactosidase activity (change in $\mathrm{OD}_{420}$ per minute) was normalized to cell density $\left(\mathrm{OD}_{650}\right)$ and was always compared to appropriate controls assayed at the same time. The values shown are averages of at least four experiments with a standard deviation of $<17 \%$, unless otherwise stated.

Immunological detection of proteins. For Western blots, cultures were grown as described in the text. Electrophoresis and protein transfer were as described previously $(7,10)$. After transfer to a Sequi-Blot polyvinylidene difluoride membrane (BioRad), blots were blocked in 5\% non-fat milk and incubated in phosphate buffered saline (PBS)-Tween (0.05\%) containing the anti-RpoS monoclonal 
antibody R12 (7). After 60 min of incubation, blots were washed in PBS-Tween, incubated for $60 \mathrm{~min}$ in PBS-Tween containing biotinylated goat anti-mouse immunoglobin, and finally incubated in PBS-

Tween containing streptavidin-conjugated horseradish peroxidase (Southern Biotechnology Associates).

Detection was by enhanced chemiluminescence (Amersham Biosciences).
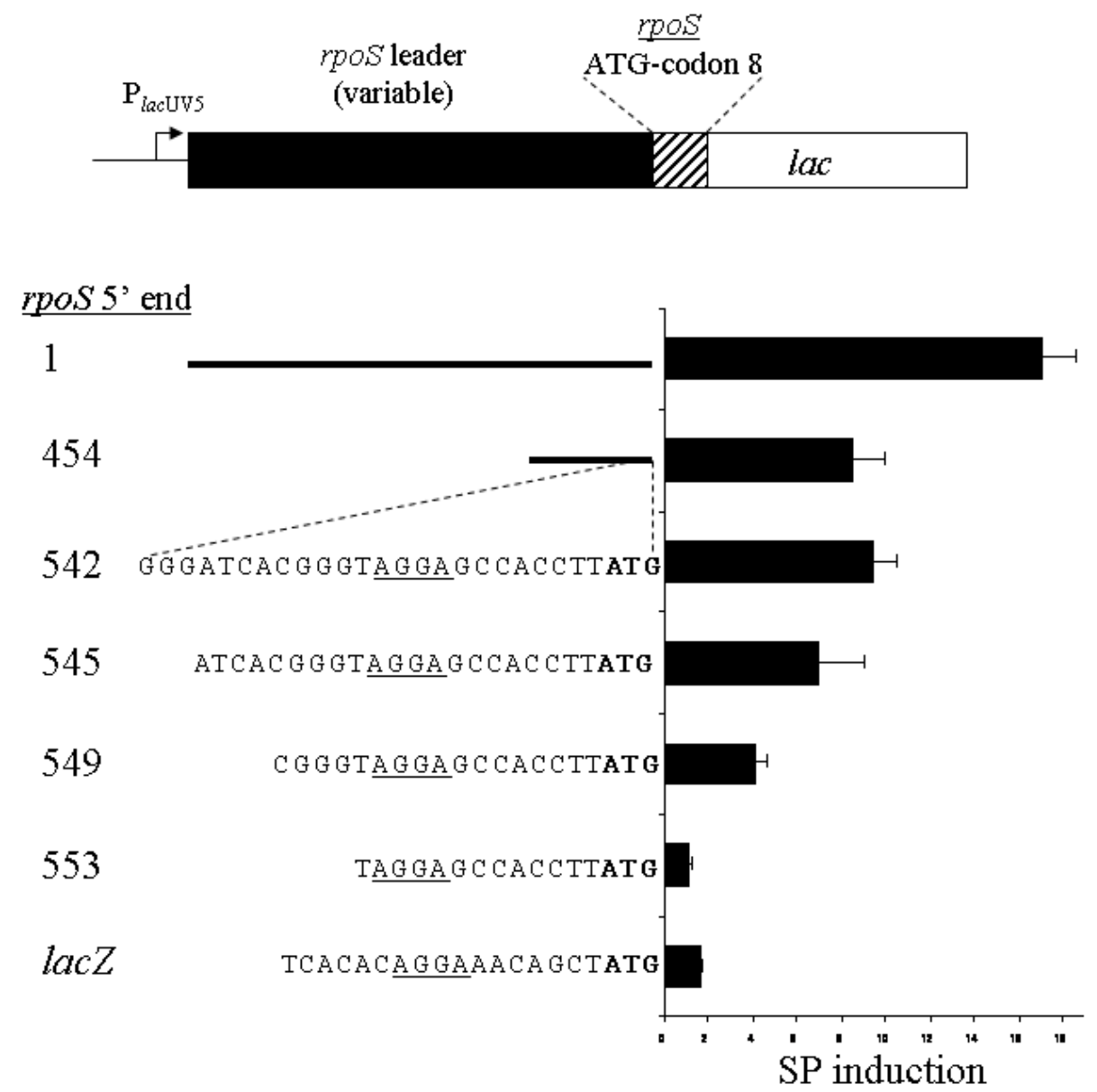

Figure 18. Stationary phase induction of $\operatorname{rpoS}$ translation relies on the ribosome-binding sequence.

The top line depicts the general fusion context. The lacUV5 promoter drives expression of a variable segment of the rpoS 5' untranslated region (rpoS leader) followed by the first 8 codons of $r p o S$. At this position, the lacZ gene is joined to $r p o S$ to form a translational fusion. Six different rpoS constructs are shown, labeled with the position of the variable upstream end of the segment. Next to each construct is the stationary phase (SP) induction ratio, obtained by dividing SP activity by the exponential-phase activity. The variable region of constructs 1 and 454 fusions are shown as black lines due to their longer size, while the broken lines indicate a magnification of $r p o S$ ribosome-binding sequence. The RBS of lacZ served as a control and is labeled lacZ. 


\section{Results}

The rpoS RBS regulates SP induction of translation. Growth of E. coli or S. enterica into SP in rich medium (LB) results in a $>30$-fold increase in expression of $r p o S$-lac [pr] (protein) and an even greater increase in RpoS activity as demonstrated by katE-lac [op] $(17,18)$. As shown by others $(21,28,33,39$, reviewed in ref 13) and confirmed by us for both $E$. coli and $S$. enterica $(17,18)$ this increase has components of both transcriptional and post-transcriptional regulation. SP induction in LB is independent of the response regulator $\mathrm{RssB} / \mathrm{SprE} / \mathrm{MviA}$ as well as the energy-dependent protease $\mathrm{ClpXP}$, which together regulate RpoS abundance under other conditions $(18,27,32,36,40)$. SP induction is also independent of the Hfq protein and likewise, the regulatory DsrA RNA (17).

A striking result from previous analysis of rpoS translation in E. coli was that most of the SP induction (at $37^{\circ} \mathrm{C}$ in $\mathrm{LB}$ ) is maintained when most of the $565 \mathrm{nt}$ rpoS leader region is deleted (17), including the antisense element that binds DsrA (25). Nearly 10-fold induction was observed for a rpoS-lac [pr] fusion expressed from $\mathrm{P}_{\text {lac }}$ 5V , that contains only $48 \mathrm{nt}$ of rpoS - $24 \mathrm{nt}$ of the RBS, which includes the Shine-Dalgarno (S.D.) sequence (GGGATCACGGGTAGGAGCCACCTTATG), followed by the first eight codons of the gene (third construct in Fig. 18, labeled 542; construct I in Fig. 19).

To further define the sequence required for translational regulation of SP induction, three additional constructs with further deletions of the rpoS leader were analyzed (Fig. 18). Cultures were 
assayed for $\beta$-galactosidase activity during exponential-phase $\left(\mathrm{OD}_{600}=0.25\right)$ and after 24 hours of growth (SP) in $\mathrm{LB}$ medium at $37^{\circ} \mathrm{C}$. The SP induction ratio (SP activity / exponential-phase activity) for each construct is reported in Fig. 18.

The construct bearing the entire $565 \mathrm{nt}$ leader region of $r p o S$, was induced 17-fold as cells grew to SP (Fig. 18, labeled 1, and Fig. 19, construct G). Constructs with sequential 5' leader deletions to within 21 nt of the rpoS initiation codon each maintained about half of this regulation, with induction ratios of seven-fold to nine-fold (Fig. 18, constructs 454, 542 and 545). Removal of an additional four nt decreased induction to an intermediate value of four-fold (Fig. 18, construct 549), while SP regulation was completely eliminated in a construct retaining only $13 \mathrm{nt}$ of the $r p o S$ leader (Fig. 18, construct 553). As a negative control for these experiments, $21 \mathrm{nt}$ of the lacZ RBS in the same fusion context (and maintaining the first eight codons of $r p o S$ ), showed a minimal 1.6-fold increase during SP (Fig. 18, $\operatorname{lacZ).}$

Conservation of $\operatorname{rpoS}$ RBS-mediated induction. The sequence of the rpoS RBS is completely conserved among several species of enteric bacteria including several strains of E. coli (including MG1655 and O157:H7), serovars of S. enterica (including Typhi and Typhimurium), Shigella flexneri and Enterobacter cloacae. To determine if RBS-mediated SP induction is specific to E. coli, we investigated the activity of construct 542 in S. enterica serovar Typhimurium. As cells grew into SP, the activity of construct 542 increased 10-fold in contrast to the 1.5-fold SP induction of the lacZ RBS (data not shown). The nearly identical induction ratios obtained with E. coli MG1655 and S. enterica serovar Typhimurium (LT2A and LT2) suggest a conserved regulatory mechanism. 


\begin{tabular}{|c|c|c|c|c|}
\hline \multirow{2}{*}{$\mathrm{P}_{\text {lacuv5 }}$} & \multirow{2}{*}{$\begin{array}{l}\text { Ribosome Binding Sequence } \\
\text { Bold }=r p o S \text { white }=\text { lacZ }\end{array}$} & \multicolumn{3}{|c|}{$\begin{array}{c}\beta \text {-galactosidase } \\
\text { activity }\end{array}$} \\
\hline & & & SP & SP/E \\
\hline \multicolumn{5}{|c|}{ Hybrid constructs } \\
\hline A. & TATAATGTGTGGAATTGGGATCACGGGTAGGAGCCACCTIATG & 8 & 68 & 8.5 \\
\hline B. & TATAATGTGTGGAATTGGGATCACGGGTAGGAGAACAGCTATG & 20 & 156 & 7.8 \\
\hline C. & TATAATGTGTGGAATTGGGATCACGGGTAGGA-AACAGCTATG & 8.2 & 51 & 6.1 \\
\hline D. & TATAATGTGTGGAATTGGGATCACGGGTAGGAGAACAGCTATG & 2.9 & 30 & 10 \\
\hline E. & TATAATGTGTGGAATT------TCACACAGGAGCCACCTTATG & 395 & 883 & 2.2 \\
\hline F. & TATAATGTGTGGAATT------TCACACAGGA-AACAGCTATG & 45 & 70 & 1.6 \\
\hline \multicolumn{5}{|c|}{ Full-length constructs $(1-565)$} \\
\hline G. & TATAATGTGTGGAATT//GATCACGGGTAGGAGCCACCTTATG & 9.6 & 169 & 17 \\
\hline $\mathrm{H}$. & TATAATGTGTGGAATT//TCAATCACACAGGA-AACAGCTATG & 67 & 103 & 1.5 \\
\hline \multicolumn{5}{|c|}{ Leader deletion constructs (542-565) } \\
\hline I. & TATAATGTGTGGAATTGGGATCACGGGTAGGAGCCACCTTATG & 11 & 105 & 9.4 \\
\hline J. & TATAATGTGTGGAATTGGGATCACCCGTAGGAGCCACCTTATG & 26 & 79 & 3 \\
\hline $\mathrm{K}$. & TATAATGTGTGGAATTGGGATCACCCGTAGGAGCCACGGTATG & 4.7 & 69 & 15 \\
\hline $\mathrm{L}$. & TATAATGTGTGGAATTGGG-TTCCACCAGGAGTGGGCACTATG & 1.0 & 13 & 13 \\
\hline
\end{tabular}

Figure 19. $\beta$-galactosidase activity of various ribosome-binding sequences.

The $\beta$-galactosidase activity of various ribosome-binding sequences (RBS), in a translational lacZ fusion context, was determined during exponential growth $(\mathrm{E})$ and during stationary phase (SP). These values were used to determine the SP induction (SP / E) of the listed constructs. All fusions are expressed from the lac UV5 promoter $\left(\mathrm{P}_{\text {lac }} \mathrm{UV5}\right)$ and a partial promoter sequence, including the -10 hexamer is underlined. The fusions are grouped into three categories: (i) hybrid constructs which contain nucleotides from both the rpoS RBS and lacZ RBS, shown in bold black font and white font, respectively; (ii) full-length constructs having the entire rpoS leader region (nucleotides 1-565), in this case a double slash mark symbolizes nt 1-541 of the native $r p o S$ transcript which are not shown; (iii) leader deletion constructs which are based on the rpoS RBS (nucleotides 542-565) with mutations affecting the RBS shown in normal font (not bold); Each RBS is linked either to the first eight codons of rpoS and then to lacZ, shown by ATG nucleotides in bold, or directly to the lacZ coding sequence (ATG in white). All strains were grown at $37^{\circ} \mathrm{C}$ in LB medium. 
the $\beta$-galactosidase activity of a construct that has just the $24 \mathrm{nt}$ of the rpoS RBS preceding native lacZ (Fig. 19, construct A). This sequence maintained an SP induction ratio of 8.5-fold, similar to the induction shown by construct 542 (Fig. 18; Fig. 19, construct I). Together with the deletion results, this clearly demonstrates that the rpoS RBS is necessary and sufficient for the nearly nine-fold increase in translation after cells enter SP.

To investigate the regulatory role of the $24 \mathrm{nt}$ rpoS RBS in the context of the entire rpoS leader, a fusion was constructed in which these bases were substituted by $21 \mathrm{nt}$ of the lacZ RBS (Fig. 19, construct $\mathrm{H})$. In this case, $\mathrm{SP}$ induction decreased to that of lacZ, 1.6-fold, compared to the 17-fold induction of the native leader region (Fig. 19, compare constructs G and H).

The $24 \mathrm{nt}$ RBS of rpoS includes a five base S.D. sequence near its center (AGGAG), bounded by 12 bases upstream and seven bases downstream. The lacZ RBS also consists of a nearly centered AGGA bordered by upstream and downstream sequence elements. We exchanged the upstream and downstream elements, to construct a panel of rpoS/lacZ RBS in the fusion contexts mentioned above (Fig. 19). In construct B, the upstream segment is from rpoS and the downstream segment is substituted from lacZ; construct $\mathrm{C}$ is identical except that it has the shorter lacZ S.D. sequence (AGGA). Both hybrid RBSs demonstrated significant SP induction similar to results obtained with the wild type rpoS RBS (Fig. 19, compare constructs A, B and C). Substitution of the downstream lacZ element is also without effect on SP induction in the context of a fusion bearing the first 8 codons of rpoS (construct D).

A quite different result is observed for substitution of the upstream element. Construct $\mathrm{E}$ contains the upstream segment from lacZ and the downstream segment from rpoS (Fig. 19). This hybrid 
RBS construct is unique among all tested herein in that its relative activity is extremely high (Fig. 19). This result appears to be due to increased translation because a corresponding transcriptional fusion demonstrates the same activity of all other transcriptional fusions tested (data not shown). Importantly, construct $\mathrm{E}$ is not regulated during growth to SP. This result was confirmed for the same hybrid RBS expressed from a mutant $\mathrm{P}_{\text {lacUv5 }}(\mathrm{T} \rightarrow \mathrm{A}$ at -12 ), which is reduced 100-fold in overall transcriptional activity (data not shown). Thus, the bases directly upstream from the S.D. sequence (rpoS nt 542-557) seem to be required for SP induction.

Testing the role of potential trans-regulators in SP induction. We considered the possibility that trans-acting factors recognize the rpoS RBS and repress activity during exponential-phase or activate translation during SP. Three genes, whose products have been implicated in control of rpoS translation, were investigated including DksA, the transiently expressed subunit of the DNA-binding HU dimer, and the RNA-binding protein Hfq $(2,7,31$, 47). Normal SP induction of construct 542 occurred in a $d k s A$, hupB or $h f q$ mutant background (data not shown). Additionally, it seems unlikely that induction is mediated by a small regulatory RNA, since most are dependent on Hfq for action (38).

We investigated whether known variations in ribosome composition as cells grow to SP confer an attraction for the rpoS RBS. The genes encoding four transiently expressed, ribosome-associated proteins YfiA, YhbH, Sra and $\operatorname{Rmf}(19,26,45)$ were individually inactivated and the SP induction of construct 542 was determined in the mutant backgrounds. In each case, SP regulation was not significantly different than wild type (data not shown). 


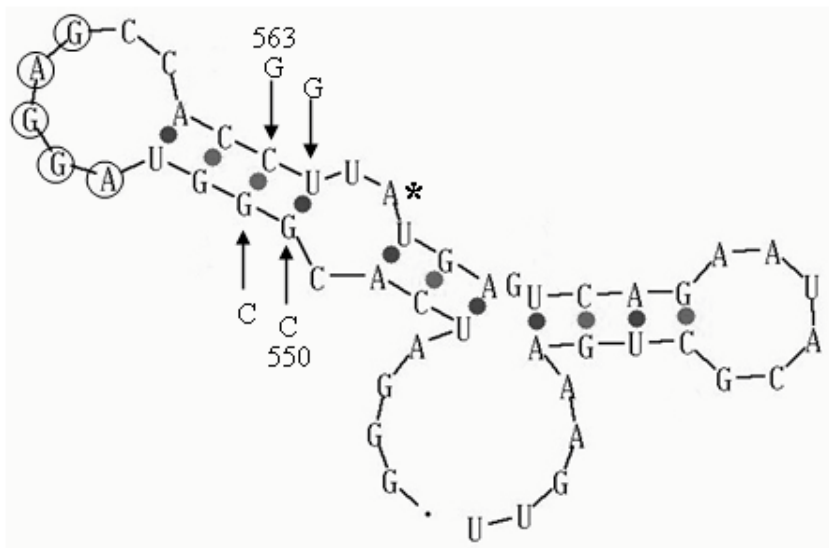

Figure 20. Predicted RNA secondary structure of the rpoS ribosome-binding region.

Mfold was used to predict the RNA secondary structure of a 48 nucleotide region of the rpoS transcript: 24 nucleotides directly preceding the rpoS start codon (labeled with an asterisk) extending to $r p o S$ codon 8 (50). The nt of the extended Shine-Dalgarno sequence of $r p o S$ are individually circled. Arrows indicate positions of directed mutations, the substituted nt are also shown, as described in the text

Secondary structure and SP induction. Another model posits that the secondary structure of the rpoS RBS acts directly as a regulatory signal. The thermodynamically favored secondary structure of construct 542 (48 nt of rpoS) was predicted using the mfold algorithm of M. Zuker (Fig. 20; 50). In this structure, the rpoS S.D. sequence (AGGAG) is positioned within a single-stranded loop flanked by a 4 bp stem. Disruption of the stem with a targeted double mutation eliminated most of the SP induction observed for the wild type rpoS RBS (Fig. 19 construct J; Fig. 20, G550C/G551C). A construct containing two compensatory mutations (Fig. 19, construct K; Fig. 20, G550C/G551C/C563G/T564G), which restore the predicted structure of the wild type RBS, was also investigated. In this case, SP induction was restored and slightly elevated compared to wild type (Fig. 19, compare constructs I, J, and $\mathrm{K})$.

To test this model further, another construct was made which reversed the sequence of the rpoS RBS while maintaining the extended S.D. sequence (Fig. 19, construct L). Remarkably, the reversed 
RBS demonstrated significant SP induction, with a ratio even higher than the wild type rpoS RBS (Fig.

19, constructs L and I). This result, along with those from the directed-mutation analyses, strongly supports the idea that the structure of the RBS, and not primary sequence, functions as a regulatory signal.

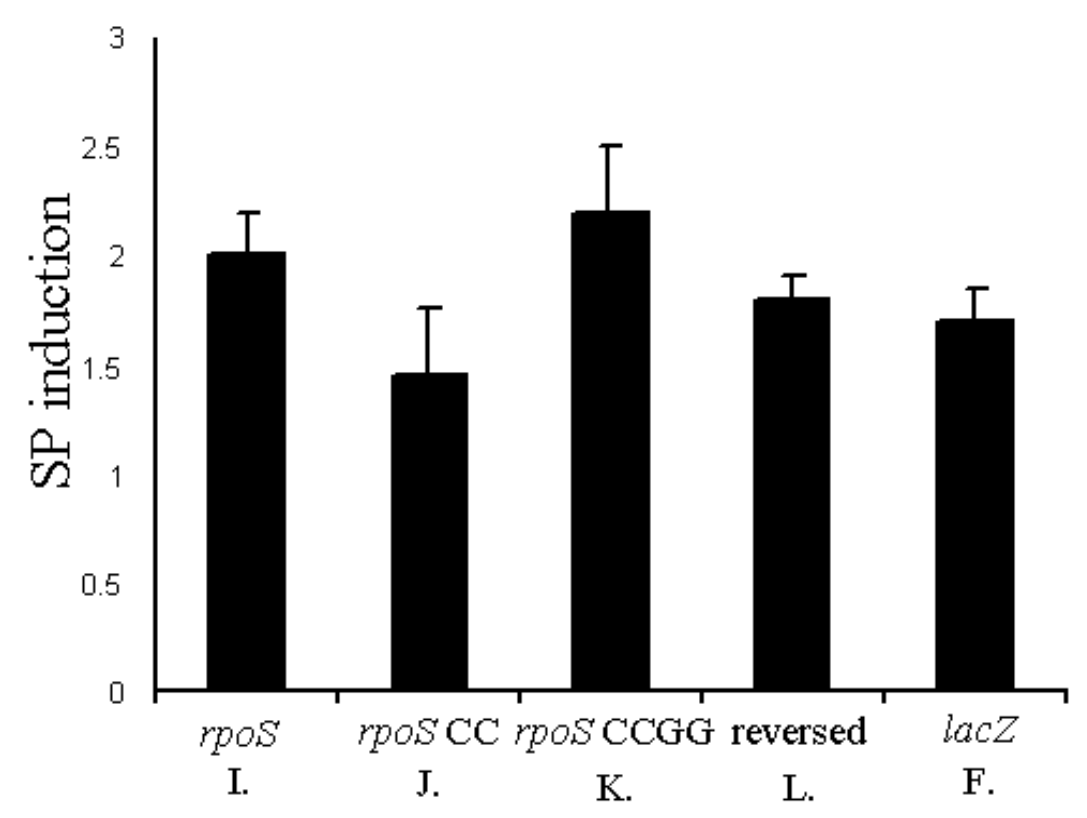

Ribosome Binding Sequence

Figure 21. Stationary phase regulation of various ribosome-binding sequences at the transcriptional level.

The stationary phase (SP) induction ratios of various transcriptional fusions containing the indicated ribosome-binding sequences were investigated. A description of the ribosome-binding sequences is given under each column and the actual sequences correspond with the letter designations of Fig. 19 . The $\beta$-galactosidase activity of each construct was determined during exponential growth (E) and SP, and the SP induction ratio $(\mathrm{SP} / \mathrm{E})$ is shown. 
RNA stability and SP induction. To investigate the possibility that differential transcript stability mediates SP induction of $r p o S$ synthesis, we constructed several strains with RBS variants in a transcriptional fusion context. All transcriptional fusions investigated demonstrated a low 1.5 to 2-fold SP induction ratio, similar to the lacZ control, regardless of translational regulation (Fig. 21). This small increase in transcriptional activity does not account for the large differences in translational induction seen among the various RBSs, indicating that altered RNA stability does not regulate SP induction.

\section{Regulation at the native rpoS locus.}

SP induction of RpoS in LB occurs at both the transcriptional and translational levels, while targeted proteolysis influences RpoS abundance but not regulation per se (18). To determine if the RBS of rpoS has a role in SP regulation in the native context, we replaced the $24 \mathrm{nt}$ sequence with the RBS of lacZ (Fig. 22A). In this background RpoS activity, as measured by the katE-lac [op] reporter, increased nearly three-fold, specifically during exponential-phase, thereby reducing SP induction (Fig. 22B). This result is supported by significantly increased RpoS protein abundance during exponential-phase (Fig. 22C, WT vs. lacZ RBS). 
A.

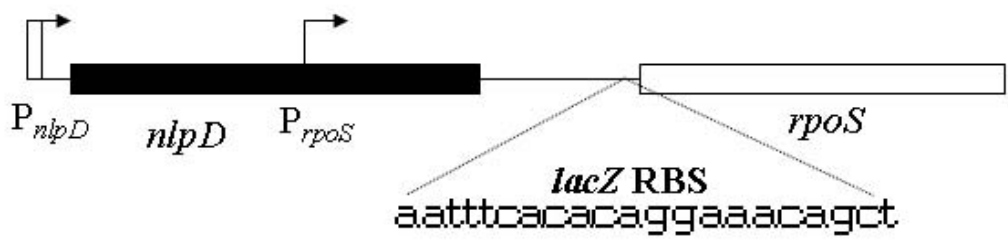

B.

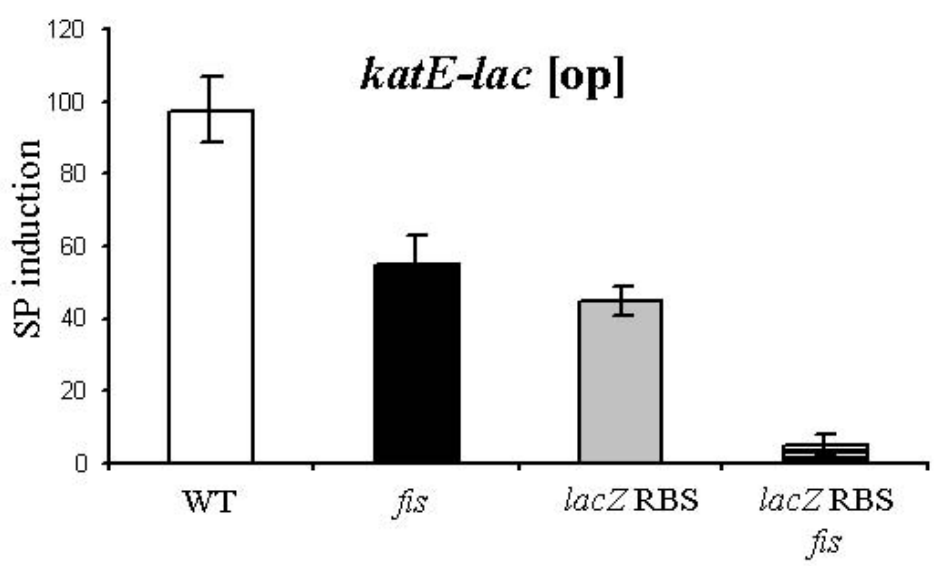

C.

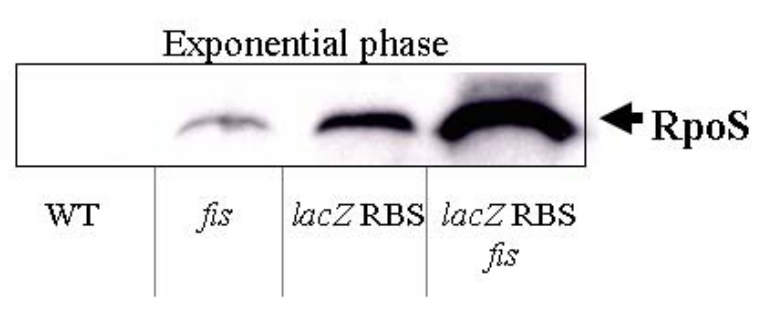

Figure 22. SP regulation of RpoS depends on the rpoS RBS and Fis.

The 24 nucleotides preceding the $r p o S$ initiation codon were replaced with the depicted lacZ RBS in the native rpoS context (A). RpoS activity during exponential growth and stationary phase was measured in a wild type background, a fis deletion mutant (fis) and in a background that contains the replacement shown in (A) with or without a fis deletion (lacZ RBS and lacZ RBS fis respectively, B). (C) Western analysis of RpoS protein during exponential growth in the indicated backgrounds. 
In S. enterica serovar Typhimurium, Fis controls SP induction of $r p o S$ transcription by binding near $\mathrm{P}_{r p o S}$ and repressing activity during exponential-phase (18). We investigated Fis regulation of RpoS in E. coli by determining the SP induction of katE-lac [op] in a fis mutant background. Due to increased expression during exponential-phase, SP induction of RpoS decreased two-fold in the fis mutant (Fig. 22B), an effect that correlates with a direct measure of RpoS abundance (Fig. 22C).

Finally, the SP induction of RpoS was determined in a E. coli background that is defective in both transcriptional control (fis mutant) and translational control (rpoS RBS replaced with that of lacZ). In this context, the near 100-fold SP induction of katE-lac [op] decreased to five-fold. This result was due to a large increase in RpoS protein during exponential-phase (Fig. 22B and 22C, compare WT vs. lacZ RBS fis).

\section{Discussion}

Regulation of RpoS in the enterics is remarkable for its diversity---both in the signals that increase the level of the protein, as well as the mechanisms by which the increase is achieved. Examples are known where regulation occurs at the level of transcription, translation, targeted proteolysis, and protein activity, as well as combinations of these (reviewed in16). Perhaps the most dramatic difference in RpoS abundance occurs between exponential-phase and SP in rich medium and is maintained by transcriptional and translational control $(17,18)$. Although this can be described as an induction or 
increase, the effects of regulatory mutants suggest that much of the control is negative, acting to restrict expression during exponential-phase.

In this study, we explore SP control of RpoS at the post-transcriptional level. Remarkably, $24 \mathrm{nt}$ of the rpoS RBS are both necessary and sufficient for a nearly nine-fold increase in translation during SP (Fig. 19). These nucleotides are highly conserved among several enteric bacteria, and the induction phenomenon occurs in S. enterica as well. Genetic evidence supports a model in which the paired structure of the rpoS RBS enforces this control (Fig. 19). However, the precise mechanism resulting in SP induction remains unclear. It is not a result of differential transcript stability (Fig. 21) or control by the reported rpoS regulators DksA or the HU dimer. Also, the RNA-binding protein Hfq is not involved and presumably, neither are small RNAs. A simple model would be that the rpoS RBS is more attractive to ribosomes during SP. If this true, then it is not mediated by known proteins associated with the ribosome specifically during SP.

The environmental stimulus that triggers RBS-mediated SP induction of rpoS translation also remains unknown, but similar to transcriptional control, regulation is only seen in rich undefined media, including LB and its individual components tryptone or yeast extract (18, data not shown). No RBSmediated SP induction occurs in minimal medium containing different carbon sources even when supplemented with amino acids and or putrescine, a polyamine reported to stimulate rpoS translation (44, data not shown). In these cases, fusion activity (Fig. 18, construct 542) is already high during exponential growth (data not shown). This media-dependent differential regulation of rpoS expression is not due to altered growth rates. 
SP induction of RpoS in rich medium depends on regulation of both transcription and translation

$(17,18)$. During exponential growth, Fis protein binds to a site near $\mathrm{P}_{r p o S}$ and blocks transcription (18).

As cells grow into SP, Fis abundance is drastically reduced and expression from $\mathrm{P}_{r p o S}$ is released from

Fis repression $(1,18)$. At the translational level, an unknown regulator acts on the structure of the rpoS

RBS and represses synthesis during exponential growth or activates it during SP (Fig. 19 and Fig. 22).

Collectively, these regulatory targets account for approximately $95 \%$ of the overall SP induction of

RpoS.

\section{Acknowledgements}

This study was supported by the Public Health Service grant GM6361. We thank individuals cited in the text for bacterial strains. 


\section{Literature Cited}

1. Ali Azam, T., A. Iwata, A. Nishimura, S. Ueda, and A. Ishihama. 1999. Growth phasedependent variation in protein composition of the Escherichia coli nucleoid. J. Bacteriol. 181:6361-6370.

2. Balandina, A., L. Claret, R. Hengge-Aronis, and J. Rouviere-Yaniv. 2001. The Escherichia coli histone-like protein HU regulates rpoS translation. Mol. Microbiol. 39:1069-1079.

3. Ball, C. A., R. Osuna, K. C. Ferguson, and R. C. Johnson. 1992. Dramatic changes in Fis levels upon nutrient upshift in Escherichia coli. J. Bacteriol. 174:8043-8056.

4. Becker, G., E. Klauck, and R. Hengge-Aronis. 2000. The response regulator RssB, a recognition factor for $\sigma^{\mathrm{S}}$ proteolysis in Escherichia coli, can act like an anti-sigma factor. Mol. Microbiol. $35: 657-66$.

5. Berkowitz, D., J. M. Hushon, H. J. Whitfield, J. Roth, and B. N. Ames. 1968. Procedure for identifying nonsense mutations. J. Bacteriol. 96:215-220.

6. Bochner, B. R., Ames, B. N. 1982. Complete analysis of cellular necleotides by two-dimensional thin layer chromatography. J. Biol. Chem. 257:9759-9769.

7. Brown, L., and T. Elliott. 1996. Efficient translation of the RpoS sigma factor in Salmonella typhimurium requires host factor I, an RNA-binding protein encoded by the $h f q$ gene. J. Bacteriol. 178:3763-3770.

8. Brown, L., and T. Elliott. 1997. Mutations that increase expression of the rpoS gene and decrease its dependence on $h f q$ function in Salmonella typhimurium. J. Bacteriol. 179:656-662.

9. Cunning, C., L. Brown, and T. Elliott. 1998. Promoter substitution and deletion analysis of upstream region required for $r p o S$ translational regulation. J. Bacteriol. 180:4564-4570.

10. Cunning, C., and T. Elliott. 1999. RpoS synthesis is growth rate regulated in Salmonella typhimurium, but its turnover is not dependent on acetyl phosphate synthesis or PTS function. J. Bacteriol. 181:4853-4862.

11. Davis, R. W., Botstein, D., Roth, J. 1980. Advanced Bacterial Genetics. Cold Spring Harbor, Cold Spring Harbor, N. Y. 
12. Elliott, T. 1992. A method for constructing single-copy lac fusions in Salmonella typhimurium and its application to the hemA-prfA operon. J. Bacteriol. 174:245-253.

13. Fang, F. C., S. J. Libby, N. A. Buchmeier, P. C. Loewen, J. Switala, J. Harwood, and D. G. Guiney. 1992. The alternative sigma factor katF (rpoS) regulates Salmonella virulence. Proc. Natl. Acad. Sci. U S A 89:11978-11982.

14. Gentry, D. R., V. J. Hernandez, L. H. Nguyen, D. B. Jensen, and M. Cashel. 1993. Synthesis of the stationary phase sigma factor $\sigma^{S}$ is positively regulated by ppGpp. J. Bacteriol. 175:79827989.

15. Gottesman, S. 2004. The small RNA regulators of Escherichia coli: roles and mechanisms. Annu. Rev. Microbiol. 58:303-328.

16. Hengge-Aronis, R. 2002. Signal transduction and regulatory mechanisms involved in control of the $\sigma^{\mathrm{S}}$ (RpoS) subunit of RNA polymerase. Microbiol. Mol. Biol. Rev. 66:373-395.

17. Hirsch, M., and T. Elliott. 2002. Role of ppGpp in rpoS stationary-phase regulation in Escherichia coli. J. Bacteriol. 184:5077-5087.

18. Hirsch, M., and T. Elliott. 2005. Fis regulates transcriptional induction of RpoS in Salmonella enterica. J. Bacteriol. 187:1568-1580.

19. Izutsu, K., C. Wada, Y. Komine, T. Sako, C. Ueguchi, S. Nakura, and A. Wada. 2001. Escherichia coli ribosome-associated protein SRA, whose copy number increases during stationary phase. J. Bacteriol. 183:2765-2773.

20. Jensen, K. F. 1993. The Escherichia coli K-12 "wild types" W3110 and MG1655 have an rph frameshift mutation that leads to pyrimidine starvation due to low pyrE expression levels. J. Bacteriol. 175:3401-3407.

21. Lange, R., and R. Hengge-Aronis. 1994. The cellular concentration of the $\sigma$ S subunit of RNA polymerase in Escherichia coli is controlled at the levels of transcription, translation, and protein stability. Genes Dev. 8:1600-1612.

22. Lease, R. A., and M. Belfort. 2000. A trans-acting RNA as a control switch in Escherichia coli: DsrA modulates function by forming alternative structures. Proc. Natl. Acad. Sci. USA 97:99199924. 
23. Lease, R. A., and S. A. Woodson. 2004. Cycling of the Sm-like protein Hfq on the DsrA small regulatory RNA. J. Mol. Biol. 344:1211-1223.

24. Linn, T., and R. St. Pierre. 1990. Improved vector system for constructing transcriptional fusions that ensures independent translation of lacZ. J. Bacteriol. 172:1077-1084.

25. Majdalani, N., C. Cunning, D. Sledjeski, T. Elliott, and S. Gottesman. 1998. DsrA RNA regulates translation of RpoS message by an anti-antisense mechanism, independent of its action as an antisilencer of transcription. Proc. Natl. Acad. Sci. U S A 95:12462-12467.

26. Maki, Y., H. Yoshida, and A. Wada. 2000. Two proteins, YfiA and YhbH, associated with resting ribosomes in stationary phase Escherichia coli. Genes Cells 5:965-974.

27. Mandel, M. J., and T. J. Silhavy. 2005. Starvation for different nutrients in Escherichia coli results in differential modulation of RpoS levels and stability. J. Bacteriol. 187:434-442.

28. McCann, M. P., C. D. Fraley, and A. Matin. 1993. The putative sigma factor KatF is regulated posttranscriptionally during carbon starvation. J. Bacteriol. 175:2143-2149.

29. Miller, J. H. 1972. Experiments in molecular genetics. Cold Spring Harbor laboratory, Cold Spring Harbor, N.Y.

30. Morita, M., M. Kanemori, H. Yanagi, and T. Yura. 1999. Heat-induced synthesis of sigma32 in Escherichia coli: structural and functional dissection of $r p o H$ mRNA secondary structure. J. Bacteriol. 181:401-1410.

31. Muffler, A., D. Fischer, S. Altuvia, G. Storz, and R. Hengge-Aronis. 1996. The response regulator RssB controls stability of the $\sigma^{\mathrm{S}}$ subunit of RNA polymerase in Escherichia coli. EMBO J. 15:1333-9.

32. Muffler, A., D. D. Traulsen, R. Lange, and R. Hengge-Aronis. 1996. Posttranscriptional osmotic regulation of the $\sigma$ S subunit of RNA polymerase in Escherichia coli. J. Bacteriol.178:1607-1613.

33. Mulvey, M. R., J. Switala, A. Borys, and P. C. Loewen. 1990. Regulation of transcription of katE and katF in Escherichia coli. J. Bacteriol. 172:6713-6720.

34. Neidhardt, F. C., Bloch, P. L., Smith, D. F. 1974. Culture medium for enterobacteria. J. Bacteriol. 119:736-747.

35. Osuna, R., D. Lienau, K. T. Hughes, and R. C. Johnson. 1995. Sequence regulation, and functions of fis in Salmonella typhimurium. J. Bacteriol. 177:2021-2032. 
36. Pratt, L. A., and T. J. Silhavy. 1996. The response regulator SprE controls the stability of RpoS. Proc. Natl. Acad. Sci. U S A 93:2488-2492.

37. Ravnum, S., and D. I. Andersson. 2001. An adenosyl-cobalamin (coenzyme-B12)-repressed translational enhancer in the cob mRNA of Salmonella typhimurium. Mol. Microbiol. 39:15851594.

38. Repoila, F., N. Majdalani, and S. Gottesman. 2003. Small non-coding RNAs, co-ordinators of adaptation processes in Escherichia coli: the RpoS paradigm. Mol. Microbiol. 48:855-861.

39. Schellhorn, H. E., and V. L. Stones. 1992. Regulation of katF and katE in Escherichia coli K12 by weak acids. J. Bacteriol. 174:4769-4776.

40. Schweder, T., K. H. Lee, O. Lomovskaya, and A. Matin. 1996. Regulation of Escherichia coli starvation sigma factor $\left(\sigma^{S}\right)$ by ClpXP protease. J. Bacteriol. 178:470-476.

41. Silhavy, T. J., Berman M. L., Enquist L. W. 1984. Experiments with gene fusions. Cold Spring Harbor Laboratory, Cold Spring Harbor, N.Y.

42. Sledjeski, D. D., A. Gupta, and S. Gottesman. 1996. The small RNA, DsrA, is essential for the low temperature expression of RpoS during exponential growth in Escherichia coli. EMBO J. 15:3993-4000.

43. Takayanagi, Y., K. Tanaka, and H. Takahashi. 1994. Structure of the 5 ' upstream region and the regulation of the rpoS gene of Escherichia coli. Mol. Gen. Genet. 243:525-31.

44. Tkachenko, A. G., and M. S. Shumkov. 2004. Role of putrescine in regulation of the $\sigma^{\mathrm{S}}$ subunit of RNA polymerase in Escherichia coli cells on transition to stationary phase. Biochemistry (Mosc) 69:876-882.

45. Wada, A., Y. Yamazaki, N. Fujita, and A. Ishihama. 1990. Structure and probable genetic location of a "ribosome modulation factor" associated with 100S ribosomes in stationary-phase Escherichia coli cells. Proc. Natl. Acad. Sci. U S A 87:2657-2661.

46. Wassarman, K. M., F. Repoila, C. Rosenow, G. Storz, and S. Gottesman. 2001. Identification of novel small RNAs using comparative genomics and microarrays. Genes Dev. 15:1637-1651.

47. Webb, C., M. Moreno, M. Wilmes-Riesenberg, R. Curtiss, 3rd, and J. W. Foster. 1999. Effects of DksA and ClpP protease on sigma S production and virulence in Salmonella typhimurium. Mol. Microbiol. 34:112-123. 
48. Weber, H., T. Polen, J. Heuveling, V. F. Wendisch, and R. Hengge. 2005. Genome-wide analysis of the general stress response network in Escherichia coli: $\sigma^{\mathrm{S}}$-dependent genes, promoters, and sigma factor selectivity. J. Bacteriol. 187:1591-1603.

49. Yu, D., H. M. Ellis, E. C. Lee, N. A. Jenkins, N. G. Copeland, and D. L. Court. 2000. An efficient recombination system for chromosome engineering in Escherichia coli. Proc. Natl. Acad. Sci. U S A 97:5978-5983.

50. Zuker, M. 2003. Mfold web server for nucleic acid folding and hybridization prediction. Nucleic Acids Res. 31:3406-3415. 
Table 8. Bacterial strains.

\begin{tabular}{|c|c|}
\hline Strain & Genotype or description \\
\hline \multicolumn{2}{|l|}{$\underline{\text { E. coli }}$} \\
\hline $\mathrm{DH} 5 \alpha$ & $\begin{array}{l}\left.\mathrm{K}-12 \mathrm{~F}^{-} \lambda^{-} \text {endA1 hsdR17( } \mathrm{r}_{\mathrm{K}}^{-} \mathrm{m}_{\mathrm{K}}^{+}\right) \text {supE44 thi-1 recA1 gyrA96 } \\
\left(\mathrm{Nal}^{\mathrm{r}}\right) \text { relA1 } \Delta(\text { lacZYA-argF }) \mathrm{U} 169(\phi 80 d \text { lacZ } \Delta \mathrm{M} 15)\end{array}$ \\
\hline TE1400 & $\mathrm{K}-12 \mathrm{~F}^{-} \lambda^{-}$araD139 $\Delta$ lacX74 galU galK $\mathrm{r}_{\mathrm{K}}^{-} \mathrm{m}_{\mathrm{K}}^{+} \mathrm{Str}^{\mathrm{R}}$ \\
\hline BW26678 & $\begin{array}{l}\text { lacI }{ }^{\mathrm{Q}} \text { rrnBTL4 } \Delta l a c Z(\mathrm{WJ} 16) \text { hsdR514 } \Delta a r a B A D(\mathrm{AH} 33) \Delta r h a B A D(\mathrm{LD} 78) / \mathrm{pKD} 46 \\
{\left[\mathrm{pSC} 101 r e p(\mathrm{Ts}) \mathrm{Amp}^{\mathrm{R}} \text { araC }^{+} \mathrm{P}_{\left.\mathrm{BAD}^{-}-\lambda r e d\right]}\right.}\end{array}$ \\
\hline CF1693 & ArelA251:: kan $\Delta$ spoT207::cat \\
\hline CF3032 & $\operatorname{argA::Tn10~\Delta relA252::kan}$ \\
\hline CF7968 & MG1655 $\Delta\left(\right.$ lacIZ) $\mathrm{rph}^{+}$ \\
\hline DDS724 & MC4100 cpsB-lac $\Delta d s r A 5$ zed::Tn10d-Tet \\
\hline DY330 & W3110 lacU169 $[\lambda$ cI857ts $\Delta($ cro-bioA $)]$ \\
\hline TE8184 & CF7968 trpDC700::putPA1303:: kan-rpoS-lac [pr] ${ }^{b}$ \\
\hline TE8197 & CF7968 argA::Tn10 trpDC700::putPA1303:: kan-rpoS-lac [pr] \\
\hline TE8199 & TE8197 $\Delta$ relA252::kan $\Delta$ spoT207::cat \\
\hline TE8222 & CF7968 argA::Tn10 trpDC700::putPA1303:: kan-rpoS-lac [op] ${ }^{a}$ \\
\hline TE8224 & TE8222 $\Delta$ relA252::kan $\Delta$ spoT207::cat \\
\hline TE8226 & CF7968 argA::Tn10 trpDC700::putPA1303:: kan-lacUV5p-rpoS-lac [pr] \\
\hline TE8228 & TE8226 $\Delta$ relA252::kan $\Delta$ spoT207::cat \\
\hline TE8230 & CF7968 argA::Tn10 trpDC700::putPA1303:: kan-lacUV5p-rpoS-lac [op] \\
\hline TE8232 & TE8230 $\Delta$ relA252::kan $\Delta$ spoT207::cat \\
\hline TE8260 & TE8184 $\Delta$ barA::tet \\
\hline TE8263 & CF7968 argA::Tn10 trpDC700::putPA1303:: kan-lacUV5p-lac [op] \\
\hline TE8265 & 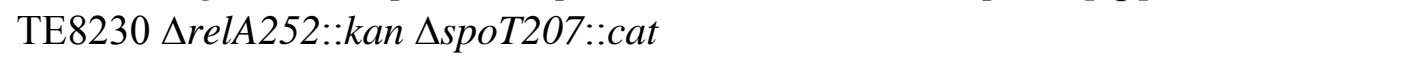 \\
\hline TE8378 & CF7968 argA::Tn10 trpDC700::putPA1303:: kan- lacUV5p -rpoS-lac [pr] (+1) \\
\hline TE8383 & TE8378 $\Delta$ relA252::kan $\Delta$ spoT207::cat \\
\hline TE8380 & CF7968 argA::Tn10 trpDC700::putPA1303:: kan-rpoS-lac [pr] (codon 8) \\
\hline TE8382 & TE8380 $\Delta$ relA252::kan $\Delta$ spoT207::cat \\
\hline TE8267 & CF7968 argA::Tn10 trpDC700::putPA1303:: kan-lacUV5p-rpoS-lac [pr] $(\Delta 1)$ \\
\hline TE8269 & TE8267 $\Delta$ relA252::kan $\Delta$ spoT207::cat \\
\hline TE8271 & CF7968 argA::Tn10 trpDC700::putPA1303:: kan-lacUV5p-rpoS-lac [pr] $(\Delta 2)$ \\
\hline TE8273 & TE8271 $\Delta$ relA252::kan $\Delta$ spoT207::cat \\
\hline TE8344 & CF7968 argA::Tn10 trpDC700::putPA1303:: kan-lacUV5p-rpoS-lac [pr] $(\Delta 3)$ \\
\hline TE8340 & TE8344 $\Delta$ relA252::kan $\Delta$ spoT207::cat \\
\hline TE8345 & CF7968 argA::Tn10 trpDC700::putPA1303:: kan -lacUV5p-rpoS-lac [pr] ( $\Delta 4)$ \\
\hline TE8341 & TE8345 $\Delta$ relA252::kan $\Delta$ spoT207::cat \\
\hline TE8275 & CF7968 argA::Tn10 trpDC700::putPA1303:: kan -rpoS-lac [pr] (C469G) \\
\hline TE8277 & TE8275 $\Delta$ relA252::kan $\Delta$ spoT207::cat \\
\hline TE8279 & CF7968 argA::Tn10 trpDC700::putPA1303:: kan -rpoS-lac [pr] (G549C) \\
\hline
\end{tabular}


TE8281 TE8279 $\Delta$ relA252::kan $\Delta$ spoT207::cat

TE8283 CF7968 argA::Tn10 trpDC700::putPA1303::kan-rpoS-lac [pr] (C469G, G549C)

TE8285 TE8283 $\Delta$ relA252::kan $\triangle$ spoT207::cat

TE8266 CF7968 trpDC700::putPA1303:: kan-lacUV5p-rpoS-lac [pr] $(\Delta 1)$

TE8316 CF7968 trpDC700::putPA1303:: kan-rpoS-lac[pr]dsrA ${ }^{+}$zed::Tn10d-Tet

TE8317 CF7968 trpDC700::putPA1303:: kan-rpoS-lac[pr] $\Delta d s r A 5$ zed::Tn10d-Tet

TE8318 TE8316 $\Delta$ relA252::kan $\Delta$ spoT207::cat

TE8319 TE8317 $\Delta$ relA252::kan $\Delta$ spoT207::cat

TE8372 TE8266 $\Delta d k s A:$ :tet

TE8270 CF7968 trpDC700::putPA1303:: kan-lacUV5p-rpoS-lac [pr] $(\Delta 2)$

TE8373 TE8270 $\Delta$ dksA::tet

TE8314 CF7968 trpDC700::putPA1303:: kan-lacUV5p-rpoS-lac [pr] ( $\Delta 3)$

TE8374 TE8314 $\Delta d k s A:$ :tet

TE8315 CF7968 trpDC700::putPA1303:: kan-lacUV5p-rpoS-lac [pr] $(\Delta 4)$

TE8375 TE8315 $\Delta$ dksA::tet

TE8363 CF7968 trpDC700::putPA1303::bla-rpoS-lac [pr]

TE8377 TE8363 hfq-1:: $\Omega-\mathrm{Km}$

TE8387 CF7968 trpDC700::putPA1303::bla-lacUV5p-rpoS-lac [pr] $(\Delta 1)$

TE8388 TE8387 hfq-1:: $\Omega-\mathrm{Km}$

TE8391 CF7968 trpDC700::putPA1303::bla-lacUV5p-rpoS-lac [pr] ( $\Delta 2)$

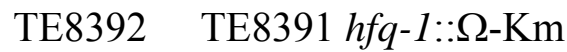

TE8402 DY330 trpDC700::putPA1303:: kan -rpoS (ClaI, codon 8)-lac [pr]

TE8403 DY330 trpDC700::putPA1303:: kan -rpoS (ClaI, codon 8)-RNAse III site-lac [op]

TE8405 TE8184 $\Delta p p k x:: t e t$

TE8419 CF7968 trpDC700::putPA1303::tet-lacUV5p-rpoS-lac [pr] (+1, codon 8)

TE8420 CF7968 trpDC700::putPA1303::tet-lacUV5p-rpoS-lac [pr] (541, codon 8)

TE8421 CF7968 trpDC700::putPA1303::tet-lacUV5p-rpoS-lac [op] (+1, codon 8)

TE8422 CF7968 trpDC700::putPA1303::tet-lacUV5p-rpoS-lac [op] (541, codon 8)

TE8439 CF7968 trpDC700::putPA1303::tetAR-lacUV5p-rpoS (454, codon 8)-lac [pr]

TE8448 CF7968 trpDC700::putPA1303::tet-lacUV5p-lacRBS-rpoS-lac [pr] (565, codon 8)

TE8483 CF7968 trpDC700::putPA1303::tetAR-lacUV5p-rpoS (541, 558)-lac (AACAGCT)rpoS (ATG, codon 8)-lac [pr]

TE8520 CF7968 trpDC700::putPA1303::tetAR-lacUV5p-(TCACAC)-rpoS (554, codon8)-lac [pr]

TE8999 CF7968 trpDC700::putPA1303::tetAR-lacUV5p-rpoS (548, codon 8)-lac [pr]

TE9024 CF7968 trpDC700::putPA1303::tetAR-lacUV5p-rpoS (541, codon 8; G550C, G551C)-lac [pr]

TE9030 CF7968 trpDC700::putPA1303::tetAR-lacUV5p-rpoS (544, codon 8)-lac [pr]

TE9036 CF7968 trpDC700::putPA1303::tetAR-lacUV5p-rpoS (552, codon 8)-lac [pr]

TE9059 DY330 trpDC700::putPA1303::kan-PlacUv5-lac [op]

TE9042 CF7968 trpDC700::putPA1303::tetAR-lacUV5p-rpoS (+1, 542)-

(TCACACAGGAACAGCT)-rpoS (ATG, codon 8)-lac [pr]

TE9145 CF7968 trpDC700::putPA1303::kan -katE-lac [op]

TE9146 CF7968 trpDC700::putPA1303::tetAR-lacUV5p-rpoS (541, 565)-lac (ATG) [pr] 
TE9200 CF7968 trpDC700::putPA1303::tetAR-lacUV5p-rpoS (541, codon 8; G550C, G551C, C563G, T564G)-lac [pr]

TE9218 CF7968 trpDC700::putPA1303::tetAR-lacUV5p-rpoS (542, 558)-(AACAGCT)-lac [op]

TE9249 CF7968 trpDC700::putPA1303::tetAR-lacUV5p-rpoS (542, 557)-(AACAGCT)-lac [op]

TE9251 CF7968 trpDC700::putPA1303::tetAR-lacUV5pGGGTTCCACCAGGAGTGGGCACT-lac [pr]

TE9268 CF7968 trpDC700::putPA1303::tetAR-lacUV5pACCACCCTGAACAGGATCAGGGCA-lac [pr]

TE9270 CF7968 trpDC700::putPA1303::tetAR-lacUV5p-ATCGATTGAGAGGATTTGA-lac [op]

TE9274 DY330 trpDC700::putPA1303:: kan-rpoS (ClaI, codon 8)-cat-lac [op]

TE9277 DY330 trpDC700::putPA1303:: kan-rpoS (ClaI, codon 8)-lac [op]

TE9284 CF7968 trpDC700::putPA1303:: kan-katE-lac [op] rpoS(540)-AATTTCACACAGGAAACAGCT-rpoS(ATG)

TE9291 CF7968 trpDC700::putPA1303::tetAR-lacUV5pACCACCCTGAACAGGATCAGGGTA-lac [op]

TE9300 CF7968 trpDC700::putPA1303::tetAR-lacUV5p-rpoS (542, codon 8)-lac [op]

TE9301 CF7968 trpDC700::putPA1303::tetAR-lacUV5p-lac (TCACACAGGAACAGCT)rpoS (ATG, codon 8)-lac [op]

TE9302 CF7968 trpDC700::putPA1303:: kan-katE-lac [op] fis::cat

TE9303 CF7968 trpDC700::putPA1303:: kan-katE-lac [op] rpoS(541)AATTTCACACAGGAAACAGCT-rpoS(ATG) fis::cat

TE9304 CF7968 trpDC700::putPA1303::tetAR-lacUV5pGGGTTCCACCAGGAGTGGGCACT-lac [op]

TE9305 CF7968 trpDC700::putPA1303::tetAR-lacUV5p-rpoS (541, codon 8; G550C, G551C, C563G, T564G)-lac [op]

TE9312 CF7968 trpDC700::putPA1303::tetAR-lacUV5p-rpoS (541, codon 8; G550C, G551C)-lac [op]

TE9335 CF7968 trpDC700::putPA1303::tetAR-lacUV5p-rpoS (542, codon 8; G550C, G551C)-lac [op]

\section{$\underline{\text { S. enterica }}$}

LT2 wild type (mviA V102G)

LT2A LT2 mviA $^{+}$

TH2285 fis-3::cat

TE315 TR5877 = hsdL6 hsdSA29 $\left(\mathrm{r}_{\mathrm{LT}}{ }^{-} \mathrm{m}_{\mathrm{LT}}{ }^{+} \mathrm{r}_{\mathrm{S}}{ }^{-} \mathrm{m}_{\mathrm{S}}{ }^{+}\right)$metA22 metE551 ilv-452 trpB2 xyl-404 rpsL120 ( Str $\left.^{\mathrm{R}}\right)$ H1-b H2-e,n,x (Fels2 $\left.{ }^{-}\right)$nml

TE6153 putPA1303::kan-katE-lac [op]

TE6675 putPA1303::kan-P tac $_{\text {-lac }[\mathrm{op}]}$

TE6676 putPA1303::kan-Placuv5-lac [op]

TE6756 LT2A putPA1303::kan-katE-lac [op]

TE6850 LT2A putPA1303::kan-katE-lac [op] clpX1::Tn10d-Cam 
TE6851 LT2A putPA1303::ka -katE-lac [op] mviA22::Tn10d-Cam

TE7304 TE315 galE putPA1303:: kan::cat-lac

TE8536 putPA1303::kan-katE-lac [op] / pKD46

TE8607 $\triangle$ cysC::tetAR

TE8698 putPA1303::kan- $\mathrm{P}_{\text {nlpD-lac [op] }}$ (13475-13053)

TE8737 rpoS1082::MudJ (codon 66)

TE8738 rpoS1082::MudJ (codon 66) / pKD46

TE8744

TE8754

TE8755

TE8758

TE8759

TE8760

TE8761

TE8764

TE8766

TE8767

TE8768

TE8770

TE8776

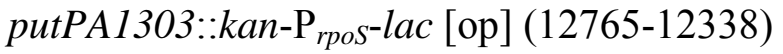

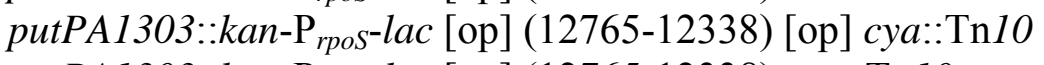

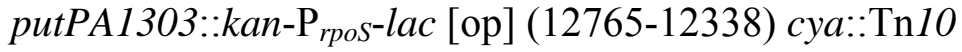

zhc-3729::Tn10d-Cam crp*-661

putPA1303::kan-Prpos-lac [op] (12765-12338) rpoS1071::Tn10d-Cam

putPA1303::kan-P lacuv5-lac [op] rpoS1071::Tn10d-Cam

putPA1303::kan-katE-lac [op] rpoS1071::Tn10d-Cam

putPA1303::kan-P ${ }_{\text {nlpD }}$-lac [op] (13475-13053) rpoS1071::Tn10d-Cam

putPA1303::kan-P rpos -lac [op] (12765-12338) fis-3::cat

putPA1303::kan-P lacuvs-lac [op] fis-3::cat

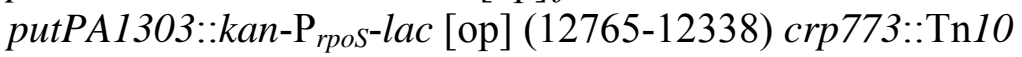

LT2 fis-3::cat

putPA1303:: kan-P rpos-lac [op] (12765-12338) crp773::Tn10

fis-3::cat

TE8787

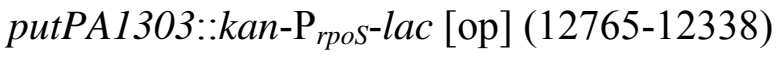

nlpD::Cam (12720)

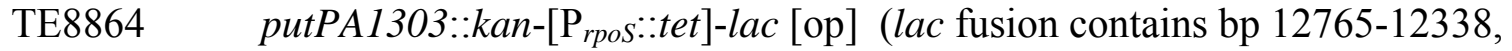
tetAR deletes 12580-12555) nlpD:: cat (12720)

TE8867 TE8864 / pKD46

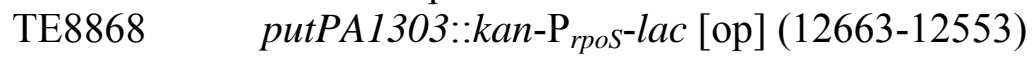

TE8869

TE8887

TE 8888

TE8895

TE8900

TE8903

putPA1303::kan- $\mathrm{P}_{\text {rpos }}$-lac [op] (12663-12553) fis-3::cat

putPA1303::kan-P rpos$^{*}$-lac [op] (12765-12338)

(* carries A12601C T12596G C12591G)

TE8887 fis-3::cat

TE8990

TE8911

TE8912

TE8991

TE8992

TE8915

TE8916

TE8917

putPA1303::kan-P $\mathrm{P}_{\text {rpos }} \Delta$-lac [op] (12765-12338, $\Delta$ removes

bp 12608-12588)

TE8895 fis-3::cat

rpoS1082::MudJ (codon 66) $\triangle$ nlpD::tetAR (13360-13003)

rpoS1082::MudJ (codon 66) $\Delta$ nlpD::tetAR (12522-12454)

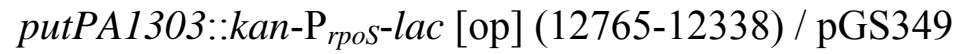

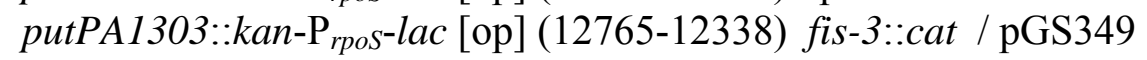

rpoS1082::MudJ (codon 66) $\triangle$ nlpD::tetAR (12584-12555)

rpoS1082::MudJ (codon 66) $\triangle$ nlpD::tetAR (13360-12555)

rpoS1082::MudJ (codon 66) $\Delta$ nlpD::tetAR (12584-12555) / pKD46

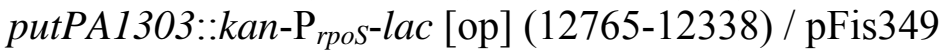

putPA1303::kan- $\mathrm{P}_{\text {rpos }}$-lac [op] (12765-12338) fis-3::cat / pFis349 


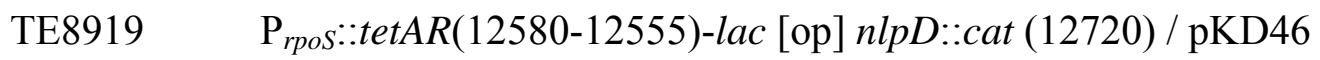

TE8925 TE8737 $\Delta \mathrm{P}_{\text {rpos }}(12584-12555)$

TE8993 TE8925 nlpD::tetAR (13360-13003)

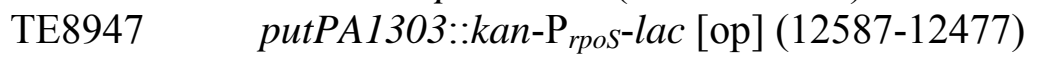

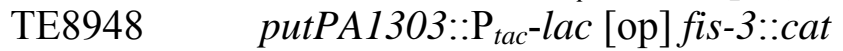

TE8949

putPA1303::kan-P rpos-lac [op] (12587-12477) fis-3::cat

TE8971

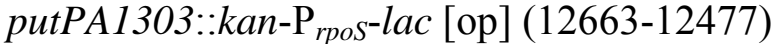

TE8972

TE9083

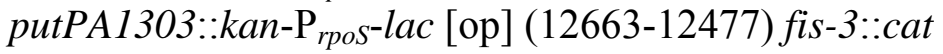

TE9096

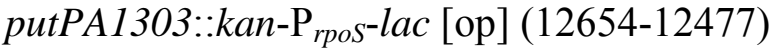

TE9195

putPA1303::kan-P rpos-lac [op] (12654-12477) fis-3::cat

TE9196

LT2A putPA1303::tetAR-lacUV5p-rpoS (542, codon 8)-lac [pr]

TE9294 LT2A putPA1303::tetAR-lacUV5p-lac (TCACACAGGAACAGCT)-rpoS (ATG, codon 8)-lac [pr]

TE9295 LT2 putPA1303::tetAR-lacUV5p-lac (TCACACAGGAACAGCT)-rpoS (ATG, codon 8)lac $[\mathrm{pr}]$

All numbering in parenthesis is in base pairs (unless otherwise indicated) relative to the first nt of the transcript originating from $\mathrm{P}_{r p o s}$. In this description, the first nt of the rpoS transcript corresponds to 2866139 of GenBank AE000111 for E. coli and nt 12589 of GenBank AE00833.1 for S. enterica serovar Typhimurium (nt upstream of the transcriptional start directly correlate with the nt designation under the given accession number). The extent of the rpoS sequence in the fusions of TE8267, TE8271, TE8344, TE8345, TE8387, TE8391 is described in the text (Chapter 2). In some cases strains were previously constructed and are noted in the text. Str ${ }^{\mathrm{r}}$, streptomycin resistance; $\mathrm{Nal}^{\mathrm{r}}$, nalidixic acid resitance.

${ }^{a}$ [op], operon (transcriptional) fusion

${ }^{b}$ [pr], protein (translational) fusion 


\section{General Discussion}

"Regulation of RpoS itself is arguably the most complicated system in bacteria"- M. Cashel (3). Individual and combined environmental cues adjust RpoS abundance at every primary level of regulation with genetic anomalies existing at the translational level (chapter 4; $5,6)$. Despite over a decade of experimentation by nearly a dozen labs, only two well established stimulus $\rightarrow$ response pathways have been elucidated: (i) carbon starvation results in RpoS induction through inhibition of ClpXP/SprE-mediated proteolysis $(9,16,21)$ and (ii) low temperature induces rpoS translation through intermolecular RNA interactions between the rpoS leader and DsrA (12). Herein, we describe a third stimulus $\rightarrow$ response pathway; SP induction of RpoS in rich medium.

Initially, we investigated the unifying hypothesis that ppGpp regulates SP induction of RpoS in E. coli (8). This was an attractive model due to three previous results: (i) conditions that induce ppGpp also induce RpoS (14), (ii) mutants deficient for ppGpp are dramatically decreased in RpoS abundance (4) and (iii) artificial overproduction of ppGpp results in RpoS induction (3). Our analysis demonstrated that SP induction occurs normally in the absence of ppGpp, although basal expression was decreased six-fold (8). In these experiments overall induction of rpoS, at both the transcriptional and translational levels was 35-fold. Surprisingly, regulation at the transcriptional level appeared to play a larger role in SP induction (15-fold) which prompted further investigations $(7,8)$. 
Several other important conclusions were drawn from this work including the intriguing result that SP induction of rpoS translation does not rely on the antisense element of the leader region (8). However, this element is necessary for adjustment of rpoS synthesis by at least two sRNAs and $\mathrm{Hfq}(11,12)$. It is likely that the role of the antisense element is to maintain low levels of synthesis during optimal conditions and regulators open the structure to increase translation regardless of growth phase. This interpretation may be applicable to DsrA, Hfq and ppGpp, all of which influence rpoS translation and require the rpoS leader for action (8). In the respective mutant backgrounds, rpoS translation was defective although SP induction was virtually unchanged (8).

Next, we focused on transcriptional regulation of rpoS, which at that time, was basically uncharacterized due to a general focus on translational models of control (6). A sequence analysis of the $\mathrm{P}_{\text {rpos }}$ region suggested putative binding sites for 17 transcriptional regulators including Fis(7). Transcriptional repression by Fis is a coherent model because an inverse correlation exists between Fis and RpoS abundance $(1,7)$. In addition, they are induced by opposite stimuli, fresh medium (Fis) and spent medium (RpoS; 2, 6). It is likely that Fis acts directly as a repressor given the site necessary for regulation in vivo is specifically bound in vitro (7). However, Fis pleiotropy in the mutant background could indirectly contribute to regulatory effects on $\mathrm{P}_{\text {rpos }}$ activity.

If Fis regulates RpoS then what regulates Fis? The generic answer is dilution into fresh medium which results in a massive induction making Fis the most abundant nucleoid-associated protein within the cell $(1,2,15)$. Fis is autoregulatory and increased Fis abundance results in 
transcriptional repression and levels decrease as a function of cell division (2). Activation of $\mathrm{P}_{\text {fis }}$ transription is tightly coupled to nutritional status including the availability of CTP, the first nt of the fis trancript (19). Additionally, $\mathrm{P}_{\text {fis }}$ activity is repressed by unfavorable growth conditions via the stringent response (20). Thus it is possible that decreased rpoS transcription in a ppGpp mutant background is due to increased Fis repression. Further investigations into the regulation of Fis expression would provide a greater understanding of the interplay of global regulators in physiological adaptation.

Our investigations regarding SP induction of RpoS at the translational level exposed a novel form of regulation (Chapter 4). Genetic dissection of the rpoS leader clearly demonstrates that the RBS mediates nearly 10 -fold induction in synthesis as cells enter SP. The importance of these nt in regulation was also shown at the native rpoS locus. The rpoS RBS is highly conserved among enteric bacteria and the induction phenomenon occurs in S. enterica as well.

The mechanism of translation induction remains elusive and is not a result of differential transcript stability, Hfq-dependent sRNAs, and protein regulators that recognize a primary sequence motif (Chapter 4). Genetic evidence heavily favors a model in which the structure of the rpoS RBS signals regulation. The structural elements of the RBS necessary for SP regulation are currently unclear, although a direct correlation was made between regulation and a singlestranded SD sequence in the predicted secondary structure (data not shown). This theory is supported by the nature of the SD in the characterized RBS structures of cbiA (single-stranded) and $r p o H$ (partially double-stranded) $(13,17)$. Of these RBSs, only cbiA signals SP induction, a result that suggests control at this level is a general phenomenon (data not shown). 
Another shocking result of our translational analysis was that lac/rpoS hybrid RBSs varied in translational efficiency up to 100-fold (Chapter 4). This was not a result of transcript stabilization (data not shown). The large translational variation in our engineered RBS is also not due to a variable spacer region, between the SD sequence and the initiation codon, or an extended RBS. However, we did show that the five nt SD sequence (AGGAG) is expressed approximately three-fold higher than AGGA in a similar context consistent with earlier reports $(10,18)$. These results demonstrate that slight nt adjustments in a RBS can dramatically affect translational efficiency and regulation.

SP regulation of RpoS induction in LB is not regulated by ClpXP-SprE proteolysis (7, 16). Although, mutants defective in protein turnover, demonstrate a three-fold increase in RpoS abundance and activity (7). It seems energetically unfavorable to make excess RpoS protein and then actively degrade it unless a quick response to an unknown stimulus is necessary. This may be the case for increases RpoS in response to carbon starvation.

\section{SP induction of RpoS in rich medium- The model}

SP induction of RpoS in rich medium depends on regulation of both transcription and translation. During exponential growth, Fis molecules bind several sites near $\mathrm{P}_{r p o s}$ and block transcription (7). As cells grow into SP, Fis abundance is low and $\mathrm{P}_{r p o s}$ expression increases $(1,7)$. At the translational level, the structure of the rpoS RBS signals SP induction by an uncharacterized 
mechanism (Chapter 4). Collectively, these regulatory targets account for approximately $95 \%$ of the overall SP induction of RpoS.

\section{Literature Cited}

1. Ali Azam, T., A. Iwata, A. Nishimura, S. Ueda, and A. Ishihama. 1999. Growth phase-dependent variation in protein composition of the Escherichia coli nucleoid. J Bacteriol 181:6361-70.

2. Ball, C. A., R. Osuna, K. C. Ferguson, and R. C. Johnson. 1992. Dramatic changes in Fis levels upon nutrient upshift in Escherichia coli. J Bacteriol 174:8043-56.

3. Brown, L., D. Gentry, T. Elliott, and M. Cashel. 2002. DksA affects ppGpp induction of RpoS at a translational level. J Bacteriol 184:4455-65.

4. Gentry, D. R., V. J. Hernandez, L. H. Nguyen, D. B. Jensen, and M. Cashel. 1993. Synthesis of the stationary-phase sigma factor sigma s is positively regulated by ppGpp. J Bacteriol 175:7982-9.

5. Gottesman, S. 2004. The small RNA regulators of Escherichia coli: roles and mechanisms*. Annu Rev Microbiol 58:303-28.

6. Hengge-Aronis, R. 2002. Signal transduction and regulatory mechanisms involved in control of the sigma(S) (RpoS) subunit of RNA polymerase. Microbiol Mol Biol Rev 66:373-95, table of contents.

7. Hirsch, M., and T. Elliott. 2005. Fis regulates transcriptional induction of RpoS in Salmonella enterica. J Bacteriol 187:1568-80.

8. Hirsch, M., and T. Elliott. 2002. Role of ppGpp in rpoS stationary-phase regulation in Escherichia coli. J Bacteriol 184:5077-87.

9. Lange, R., and R. Hengge-Aronis. 1994. The cellular concentration of the sigma S subunit of RNA polymerase in Escherichia coli is controlled at the levels of transcription, translation, and protein stability. Genes Dev 8:1600-12.

10. Lithwick, G., and H. Margalit. 2003. Hierarchy of sequence-dependent features associated with prokaryotic translation. Genome Res 13:2665-73. 
11. Majdalani, N., S. Chen, J. Murrow, K. St John, and S. Gottesman. 2001. Regulation of RpoS by a novel small RNA: the characterization of RprA. Mol Microbiol 39:138294.

12. Majdalani, N., C. Cunning, D. Sledjeski, T. Elliott, and S. Gottesman. 1998. DsrA RNA regulates translation of RpoS message by an anti-antisense mechanism, independent of its action as an antisilencer of transcription. Proc Natl Acad Sci U S A 95:12462-7.

13. Morita, M., M. Kanemori, H. Yanagi, and T. Yura. 1999. Heat-induced synthesis of sigma32 in Escherichia coli: structural and functional dissection of $r p o H$ mRNA secondary structure. J Bacteriol 181:401-10.

14. Neidhardt, F. C. 1996. Escherichia coli and Salmonella, second ed, vol. 1. ASM Press, Washington, DC.

15. Osuna, R., D. Lienau, K. T. Hughes, and R. C. Johnson. 1995. Sequence, regulation, and functions of fis in Salmonella typhimurium. J Bacteriol 177:2021-32.

16. Pratt, L. A., and T. J. Silhavy. 1996. The response regulator SprE controls the stability of RpoS. Proc Natl Acad Sci U S A 93:2488-92.

17. Ravnum, S., and D. I. Andersson. 2001. An adenosyl-cobalamin (coenzyme-B12)repressed translational enhancer in the cob mRNA of Salmonella typhimurium. Mol Microbiol 39:1585-94.

18. Ringquist, S., S. Shinedling, D. Barrick, L. Green, J. Binkley, G. D. Stormo, and L. Gold. 1992. Translation initiation in Escherichia coli: sequences within the ribosomebinding site. Mol Microbiol 6:1219-29.

19. Walker, K. A., P. Mallik, T. S. Pratt, and R. Osuna. 2004. The Escherichia coli Fis promoter is regulated by changes in the levels of its transcription initiation nucleotide CTP. J Biol Chem 279:50818-28.

20. Walker, K. A., and R. Osuna. 2002. Factors affecting start site selection at the Escherichia coli fis promoter. J Bacteriol 184:4783-91. 
21. Zgurskaya, H. I., M. Keyhan, and A. Matin. 1997. The sigma S level in starving Escherichia coli cells increases solely as a result of its increased stability, despite decreased synthesis. Mol Microbiol 24:643-51. 